\title{
RADIATION FIELDS, SCATTERING AND INVERSE SCATTERING ON ASYMPTOTICALLY HYPERBOLIC MANIFOLDS
}

\author{
ANTÔNIO SÁ BARRETO
}

\section{INTRODUCTION}

The purpose of this article is to define the radiation fields on asymptotically hyperbolic manifolds and to use them to study scattering theory. The radiation fields on $\mathbb{R}^{n}$ and on asymptotically Euclidean manifolds were introduced by F.G. Friedlander in a series of papers starting in the early 1960's 10, 11, 12, 13, 14. His program of using the radiation fields to obtain the scattering matrix in that general setting was completed in [48. Here we carry out the analogous construction on asymptotically hyperbolic manifolds. After defining the radiation fields, we use them to give a unitary translation representation of the wave group and to obtain the scattering matrix for such manifolds. As an application, we use them to study the inverse problem of determining the manifold and the metric from the scattering matrix at all energies.

Asymptotically hyperbolic manifolds are smooth compact manifolds with boundary equipped with a complete metric that resembles the hyperbolic space near the boundary. The basic examples of such manifolds are the hyperbolic space and its quotients by certain discrete group actions, see 43], but any $C^{\infty}$ compact manifold with boundary can be equipped with such a metric.

There is a history of interest in scattering theory for this class of manifolds, motivated by several problems of mathematics and physics, which goes back to the work of Fadeev and Pavlov [8], followed by Lax and Phillips [36, 37, and later by several people, see for example [2, 6, 20, 28, 39, 43, 45, and references cited there. More recently there has been interest in this class of manifolds in connection to conformal field theory, see [9, 15, 16] and references cited there.

Mazzeo, Mazzeo and Melrose [39, 40, 43] first studied the spectral and scattering theory of the Laplacian in this general setting and gave a thorough description of the resolvent and its meromorphic continuation. Their methods have been applied in [6, 16, 17, 20, 28, to study the scattering matrix starting from a careful understanding of the structure of the solutions to the Schrödinger equation on a neighborhood of infinity.

We will develop the scattering theory for this class of manifolds using a dynamical approach in the style of Lax and Phillips [35, 36, 37, but we do this by following Friedlander [10, 11, 12, 13, 14].

We define the forward radiation field for asymptotically hyperbolic manifolds as the limit, as times goes to infinity, of the forward fundamental solution of the wave operator along certain light rays. The backward radiation field is defined by reversing the time direction. These are generalizations of the LaxPhillips transform [33, 36] to this class of manifolds. We will show that this leads to a unitary translation representation of the wave group and a dynamical definition of the scattering matrix as in [35].

In section [ 6] we establish the connection of the radiation fields and the Poisson operator, which in this context is also called the Eisenstein function. This is then used to show that the stationary (via Schrödinger's equation) and the dynamical (via radiation fields) definitions of the scattering matrix are equivalent.

2000 Mathematics Subject Classification. Primary 81U40, Secondary 35P25.

Supported by the NSF under grant DMS-0140657. 
To show the existence of the radiation fields, we adapt the techniques of 13,14 to this setting. To connect the radiation fields, the Poisson operator and the scattering matrix, we use the construction of the resolvent of the Laplacian due to Mazzeo and Melrose 43 and the construction of the Eisenstein function from the resolvent from [20, 28].

In section 3 as an example, we compute the forward radiation field for the three dimensional hyperbolic space $\mathbb{H}^{3}$ and show that it is given by the Lax-Phillips transform, which is based on the horocyclic Radon transform. The case on $\mathbb{H}^{n}$ is treated in 34 .

In section 17 we prove a precise support theorem - in the terminology of Helgason [23, 24] - for the radiation fields. Theorem 7.1 below generalizes to this setting a theorem of Lax-Phillips, Theorem 3.13 of 36, see also 33, obtained for the horocyclic Radon transform. Helgason 21] proved this result for compactly supported functions, but in more general symmetric spaces. Theorem 7.1 below extends this to asymptotically hyperbolic manifolds, where the horocyclic Radon transform is replaced by the radiation field. This can be thought of as a result in control theory which, roughly speaking, says that the support of a function is controlled by the support of its radiation field.

Radon type transforms are often used to study properties of solutions of hyperbolic equation, but here we use the equation to study support properties of the radiation field. This allows the use of uniqueness theorems for partial differential equations to establish support properties of these transforms. The main ingredients of the proof of the support theorem are Hörmander's uniqueness theorem for the Cauchy problem, see Theorem 28.3.4 of 26, and two of its refinements, one due to Alinhac [1] and another one which is due to Tataru [4]. The study of support properties of Radon transforms is a topic of interest in its own, see for example [23, 33] and references cited there.

In section 8 we use the characterization of the scattering matrix through the radiation fields and the boundary control method of Belishev 4, see also [5, 29, 30, and the book by Katchalov, Kurylev and Lassas 31, to study the inverse problem of determining the manifold and the metric from the scattering matrix at all energies. We prove that the scattering matrix of an asymptotically hyperbolic manifold determines the manifold and the metric up to invariants.

\section{Asymptotically Hyperbolic Manifolds and Radiation Fields}

A smooth compact manifold $X$ with boundary, $\partial X$, is called asymptotically hyperbolic, see [43], when

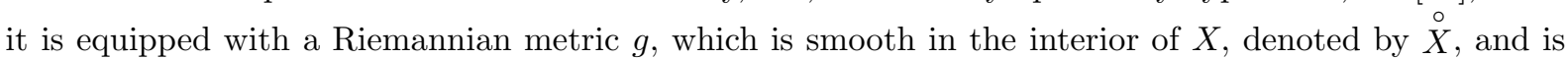
such that for a smooth defining function $x$ of $\partial X$, that is $x>0$ in the interior of $X, x=0$ on $\partial X$ and $d x \neq 0$ at $\partial X$

$$
x^{2} g=H,
$$

is a smooth Riemannian metric on $X$ non-degenerate up to $\partial X$. Furthermore we assume that

$$
|d x|_{H}=1 \quad \text { at } \partial X
$$

It can be shown, see 39 43, that under these assumptions, the sectional curvature approaches -1 at $\partial X$.

Observe that $g$ determines $x$ and $H$ only up to a positive factor. Hence $g$ induces a conformal structure at $\partial X$.

Throughout this paper, $X$ denotes a $n+1$ dimensional smooth compact manifold with boundary, and $n \geq 1$. $g$ will be a Riemannian metric on $X$ satisfying (2.1) and $\Delta$ will denote the (positive) Laplace operator with respect to the metric $g$. 
As stated in 44, see 28] for a proof in this general setting, fixed a defining function $x$ of $\partial X$, then for all $f \in C^{\infty}(\partial X)$ and $\lambda \in \mathbb{R}, \lambda \neq 0$, there exists a unique $u \in C^{\infty}(\stackrel{\circ}{X})$ satisfying

$$
\begin{gathered}
\left(\Delta-\lambda^{2}-\frac{n^{2}}{4}\right) u=0 \text { in } \stackrel{\circ}{X}, \\
u=x^{i \lambda+\frac{n}{2}} f_{+}+x^{-i \lambda+\frac{n}{2}} f_{-}, \quad f_{ \pm} \in C^{\infty}(\bar{X}),\left.\quad f_{+}\right|_{\partial X}=f .
\end{gathered}
$$

This leads to the stationary definition of the scattering matrix at energy $\lambda \neq 0$, see for example [20] 28, 44, as the operator

$$
\begin{gathered}
A(\lambda): C^{\infty}(\partial X) \longrightarrow C^{\infty}(\partial X) \\
\left.f \longmapsto f_{-}\right|_{\partial X} .
\end{gathered}
$$

As pointed out in 44, the expansion (2.2) gives two parametrizations, corresponding to $\pm \lambda$, of the generalized eigenspace of $\Delta-\frac{n^{2}}{4}$ with eigenvalue $\lambda^{2}$ by distributions on $\partial X$. The scattering matrix is the operator that intertwines them.

Notice that if $\Psi$ is a diffeomorphism of $\bar{X}$, fixing $x$ and $d x$ on $\partial X$, then the scattering matrix will be invariant under the pull back of the metric by $\Psi$. Moreover, this definition of the scattering matrix depends on the choice of the function $x$. It can be invariantly defined as acting on appropriate bundles, see for example 28 .

It is shown in [28] and [15], that if $g$ satisfies (2.1), and fixed a representative $h_{0}$ of the conformal class of $g$ at $\partial X$, there exists $\epsilon>0$ and a unique product structure $X \sim[0, \epsilon) \times \partial X$ in which

$$
g=\frac{d x^{2}}{x^{2}}+\frac{h(x, y, d y)}{x^{2}}, \quad h_{0}=h(0, y, d y) .
$$

We will fix such a decomposition, and from now on $x \in C^{\infty}(X)$ will be as in (2.4). This is equivalent to fixing a conformal representative of $\left.x^{2} g\right|_{\partial X}$. We will also work with the $A(\lambda)$ defined by (2.3) where $x$ is given by (2.4).

Here, as in [13 14, we will use the wave equation to define the radiation fields and arrive at an equivalent definition of the scattering matrix.

We will prove

Theorem 2.1. For $f_{1}, f_{2} \in C_{0}^{\infty}(\stackrel{\circ}{X})$, compactly supported in the interior of $X$, let $u(t, z) \in C^{\infty}\left(\mathbb{R}_{+} \times \stackrel{\circ}{X}\right)$ satisfy

$$
\begin{gathered}
\left(D_{t}^{2}-\Delta+\frac{n^{2}}{4}\right) u(t, z)=0, \text { on } \mathbb{R}_{+} \times \stackrel{\circ}{X}, \\
u(0, z)=f_{1}(z), \quad D_{t} u(0, z)=f_{2}(z) .
\end{gathered}
$$

Let $z=(x, y) \in(0, \epsilon) \times \partial X$ be local coordinates near $\partial X$ in which (2.4) hold. Then there exist $w_{k} \in$ $C^{\infty}(\mathbb{R} \times \partial X)$, such that

$$
x^{-\frac{n}{2}} u(s-\log x, x, y) \sim \sum_{k=0}^{\infty} w_{k}(s, y) x^{k} .
$$

Clearly $w_{k}(s, y)$ depends on the choice of $x$, and we make no attempt to define a bundle where it would be invariant. We refer the reader to Lemma 2.2 of [15] for the relationship between two functions that satisfy (2.4) corresponding to two different conformal representatives.

Theorem 2.1 defines a map

$$
\begin{gathered}
\mathcal{R}_{+}: C_{0}^{\infty}(\stackrel{\circ}{X}) \times C_{0}^{\infty}(\stackrel{\circ}{X}) \longrightarrow C^{\infty}(\mathbb{R} \times \partial X) \\
\mathcal{R}_{+}(f)(s, y)=\left.x^{-\frac{n}{2}} D_{t} u(s-\log x, x, y)\right|_{x=0}=D_{s} w_{0}(s, y),
\end{gathered}
$$


which will be called the forward radiation field.

Similarly one can prove that if $u_{-}$satisfies $(2.5)$ in $\mathbb{R}_{-} \times \stackrel{\circ}{X}$ then

$$
\lim _{x \rightarrow 0} x^{-\frac{n}{2}} u_{-}(s+\log x, x, y)=w_{-}(s, y)
$$

exists, and thus define the backward radiation field

$$
\begin{gathered}
\mathcal{R}_{-}: C_{0}^{\infty}(\stackrel{\circ}{X}) \times C_{0}^{\infty}(\stackrel{\circ}{X}) \longrightarrow C^{\infty}(\mathbb{R} \times \partial X) \\
\mathcal{R}_{-}(f)(s, y)=\left.x^{-\frac{n}{2}} u_{-}(s+\log x, x, y)\right|_{x=0}=D_{s} w_{-}(s, y) .
\end{gathered}
$$

Finally we remark that, since the Lorentzian metric associated to $g$ is

$$
\sigma=d t^{2}-\frac{d x^{2}}{x^{2}}-\frac{h(x, y, d y)}{x^{2}}=d(t-\log x) d(t+\log x)-\frac{h(x, y, d y)}{x^{2}},
$$

the surfaces

$$
\{t-\log x=C\}, \quad\{t+\log x=C\}
$$

are characteristic for the wave operator, and thus a point $\left(t^{\prime}, z^{\prime}\right), z^{\prime}=\left(x^{\prime}, y^{\prime}\right)$, has a past domain of dependence, $\Delta^{-}\left(t^{\prime}, z^{\prime}\right)$ satisfying

$$
\Delta^{-}\left(t^{\prime}, z^{\prime}\right) \subset\left\{(t, x, y): t-\log x \geq t^{\prime}-\log x^{\prime}, \quad t+\log x \leq t^{\prime}+\log x^{\prime}\right\} .
$$

\section{The Three Dimensional Space with Constant Negative Curvature}

In this section, as an example, we study the particular case of the hyperbolic space

$$
\mathbb{H}^{3}=\left\{(x, y): y \in \mathbb{R}^{2}, x \in \mathbb{R}, \quad x>0\right\}, \text { with the metric } g=\frac{d x^{2}}{x^{2}}+\frac{|d y|^{2}}{x^{2}} .
$$

In this case the radiation fields can be explicitly computed. The formulæ obtained in [32], see also [22], can be used in the same way to compute the radiation fields in $\mathbb{H}^{n}$. This is done in [34.

For convenience, we will work in the non-compact model, which does not quite fit the framework of section 1 but it is isometric to the compact model given by the interior of the ball with Poincaré's metric $X=\mathbb{B}^{3}=\left\{z \in \mathbb{R}^{3}:|z|<1\right\}$ and $g=\frac{4|d z|^{2}}{\left(1-|z|^{2}\right)^{2}}$.

For $z \in \mathbb{H}^{3}$, let $S(z, t)$ denote the set of points in $\mathbb{H}^{3}$ whose geodesic distance to $z$ is $t$. Let $A(t)$ be the area of $S(z, t)$, which is independent of $z$. Given $f \in C_{0}^{\infty}\left(\mathbb{H}^{3}\right)$, supported in the interior of $\mathbb{H}^{3}$, let

$$
M(f, t, z)=\frac{1}{A(t)} \int_{S(z, t)} f(y) d \sigma_{y}
$$

be the mean of $f$ over the sphere $S(z, t)$.

According to [22, 32, the solution to (2.5) with $f_{1}=0$ and $f_{2}=f$ is given by

$$
u(t, z)=(\sinh t) M(f, t, z) .
$$

Therefore, the forward fundamental solution is, for $t>0$,

$$
U\left(t, z, z^{\prime}\right)=\frac{\sinh t}{A(t)} \delta\left(t-d\left(z^{\prime}, z\right)\right),
$$

where the distance is given by

$$
\cosh d\left(z, z^{\prime}\right)=\frac{x^{2}+{x^{\prime}}^{2}+\left|y-y^{\prime}\right|^{2}}{2 x x^{\prime}} .
$$

Then the sphere in the hyperbolic metric centered at $(x, y)$ with radius $t$ corresponds to the Euclidean sphere with center $(x \cosh t, y)$ and radius $x \sinh t$. We find that $A(t)=4 \pi \sinh ^{2} t$, and hence

$$
U\left(t, z, z^{\prime}\right)=\frac{1}{4 \pi \sinh t} \delta\left(t-d\left(z, z^{\prime}\right)\right) .
$$


But $\lim _{x \rightarrow 0} x \sinh (s-\log x)=\lim _{x \rightarrow 0} x \cosh (s-\log x)=\frac{e^{s}}{2}$. So the Schwartz kernel of $\mathcal{R}_{+}$is

$$
\mathcal{R}_{+}\left(s, y, z^{\prime}\right)=\lim _{x \rightarrow 0} x^{-1} \frac{\partial}{\partial s} U\left(s-\log x, z, z^{\prime}\right)=\frac{\partial}{\partial s}\left(\frac{1}{4 \pi e^{s}} \delta\left(\frac{e^{s}}{2}-d_{e}\left[\left(\frac{e^{s}}{2}, y\right), z^{\prime}\right]\right)\right)
$$

where $d_{e}$ denotes the distance in the Euclidean metric.

So, for $f \in C_{0}^{\infty}\left(\mathbb{H}^{3}\right)$, one obtains $\mathcal{R}_{+} f(s, y)$ by integrating $f$ over the surface of the Euclidean sphere with center $\left(\frac{e^{s}}{2}, y\right)$ and radius $\frac{e^{s}}{2}$, with respect to the measure induced by the metric $g$. This sphere is tangent to $\{x=0\}$ at $(0, y)$ and the integration of $f$ on those spheres is known as the horocyclic Radon transform, see for example [24]. The transformation given by (3.4) is called the Lax-Phillips transform, see 33 .

\section{The Proof of Theorem 2.1}

In this section we work with the forward radiation field and we will drop the index \pm from the notation. We will also denote

$$
h(x, y, d y)=\sum_{i, j=1}^{n} h_{i j}(x, y) d y_{i} d y_{j}, \quad|h|=\operatorname{det}\left(h_{i j}\right), \text { and } h^{-1}=\left(h^{i j}\right), \text { the inverse of the matrix } h_{i j} .
$$

Proof. Without loss of generality, we may assume that $f_{1}=0$ and that $f_{2}$ is compactly supported in $\left\{x>x_{0}\right\}$, with $x_{0}$ small enough. Hence $u(t, x, y) \in C^{\infty}(\mathbb{R} \times(0, \epsilon) \times \partial X)$ satisfies

$$
\begin{gathered}
\left(D_{t}^{2}-\Delta+\frac{n^{2}}{4}\right) u(t, x, y)=0, \\
u(0, x, y)=0, \quad D_{t} u(0, x, y)=f(x, y) .
\end{gathered}
$$

Here we are considering the solution in $t \in \mathbb{R}$, and in this case the solution $u$ is odd in $t$. Moreover (2.8) and the finite speed of propagation guarantee that for $x$ small,

$$
u=0 \quad \text { if } \quad \log x-\log x_{0}<t<\log x_{0}-\log x, \quad x<x_{0}<\epsilon .
$$

We will show that

$$
v(s, x, y)=x^{-\frac{n}{2}} u(s-\log x, x, y)
$$

is smooth up to $x=0$. We denote

$$
P=x^{-\frac{n}{2}-1}\left(D_{t}^{2}-\Delta+\frac{n^{2}}{4}\right) x^{\frac{n}{2}}
$$

and substitute $t=s-\log x$. Then using (4.2), we find

$$
\begin{gathered}
P v(s, x, y)=0, \text { in } \mathbb{R} \times(0, \epsilon) \times \partial X, \quad P=\frac{\partial}{\partial x}\left(2 \frac{\partial}{\partial s}+x \frac{\partial}{\partial x}\right)+x \Delta_{h}+A \frac{\partial}{\partial s}+A x \frac{\partial}{\partial x}+\frac{n}{2} A \\
v(\log x, x, y)=0, \quad D_{s} v(\log x, x, y)=x^{-\frac{n}{2}} f(x, y)
\end{gathered}
$$

Here $\Delta_{h}$ is the Laplacian on $\partial X$ associated with the metric $h(x, y, d y)$, which in local coordinates is $\Delta_{h}=\frac{1}{|h|^{\frac{1}{2}}} \sum_{i, j=1}^{n} \frac{\partial}{\partial y_{i}}\left(|h|^{\frac{1}{2}} h^{i j} \frac{\partial}{\partial y_{j}}\right)$, and $A=\frac{1}{2}|h|^{-1} \frac{\partial|h|}{\partial x}$.

Equation (4.3) implies

$$
v=0 \text { for } 2 \log x-\log x_{0}<s<\log x_{0}, \quad x<x_{0}<\epsilon .
$$

The operator $P$ is not strictly hyperbolic at $x=0$, so the argument of Friedlander 14, which is based on a theorem of Leray, cannot be used directly. We will refine the method of 13 and obtain energy estimates which hold uniformly up to $\{x=0\}$. 
It is worth observing that if the tensor $h(x, y, d y)$ is an even function of $x$, then after setting $r=x^{2}$, the operator $r^{-\frac{1}{2}} P$ is smooth and strictly hyperbolic. So Friedlander's method can be applied directly. Such metrics have been recently studied by Guillarmou in 17 and include the case where the metric has constant curvature near $\partial X$, see [19]. In the general case this trick does not work because the resulting operator would not be smooth at $r=0$.

In section 7 we will need to understand the behavior of the forward radiation field as $s \rightarrow-\infty$, so we will compactify the problem and obtain uniform estimates as $s \rightarrow-\infty$. So we make the change of variables

$$
s=2 \log t^{\prime}, \quad x=x^{\prime} t^{\prime}
$$

This choice of coordinates is designed to do two things: first it transforms the operator $\frac{\partial}{\partial x}\left(2 \frac{\partial}{\partial s}+x \frac{\partial}{\partial x}\right)$ into $\frac{\partial}{\partial x^{\prime}} \frac{\partial}{\partial t^{\prime}}$, and secondly it compactifies the half-line $(-\infty, 0]$. Thus let

$$
V\left(x^{\prime}, t^{\prime}, y\right)=v\left(2 \log t^{\prime}, x^{\prime} t^{\prime}, y\right)=\left(x^{\prime} t^{\prime}\right)^{-\frac{n}{2}} u\left(\log \left(\frac{t^{\prime}}{x^{\prime}}\right), x^{\prime} t^{\prime}, y\right) .
$$

Then $V$ is smooth in $t^{\prime}>0, x^{\prime}>0$ and, as $u$ is odd in $t, V\left(x^{\prime}, t^{\prime}, y\right)=-V\left(t^{\prime}, x^{\prime}, y\right)$. Moreover for

$$
\begin{gathered}
P^{\prime}=\frac{\partial}{\partial x^{\prime}} \frac{\partial}{\partial t^{\prime}}+x^{\prime} t^{\prime} \Delta_{h}+\frac{1}{2} A\left(x^{\prime} t^{\prime}, y\right)\left(t^{\prime} \frac{\partial}{\partial t^{\prime}}+x^{\prime} \frac{\partial}{\partial x^{\prime}}\right)+\frac{n}{2} A\left(x^{\prime} t^{\prime}, y\right), \\
P^{\prime} V=0, x^{\prime}>0, t^{\prime}>0, \\
V\left(x^{\prime}, x^{\prime}, y\right)=0, \quad \frac{\partial}{\partial x^{\prime}} V\left(x^{\prime}, x^{\prime}, y\right)=-x^{\prime-n-1} f\left(x^{\prime 2}, y\right) .
\end{gathered}
$$

Here $\Delta_{h}$ is the Laplacian with respect to the metric $h\left(x^{\prime} t^{\prime}, y, d y\right)$ which in local coordinates is $\Delta_{h}=$ $\frac{1}{\left|h\left(x^{\prime} t^{\prime}, y\right)\right|^{\frac{1}{2}}} \sum_{i, j} \frac{\partial}{\partial y_{i}}\left(\left|h\left(x^{\prime} t^{\prime}, y\right)\right|^{\frac{1}{2}} h^{i j}\left(x^{\prime} t^{\prime}, y\right) \frac{\partial}{\partial y_{j}}\right)$.

The support properties of $v$ given in (4.6) translate into

$$
V\left(x^{\prime}, t^{\prime}, y\right)=0 \quad \text { if } \quad x^{\prime}<\sqrt{x_{0}}, \quad t^{\prime}<\sqrt{x_{0}} .
$$

The coefficients of $P^{\prime}$ are smooth up to $\left\{x^{\prime}=0\right\} \cup\left\{t^{\prime}=0\right\}$ and therefore can be extended smoothly, although not uniquely, to a neighborhood $\left\{\left|x^{\prime}\right|<\sqrt{x_{0}}\right\} \cup\left\{\left|t^{\prime}\right|<\sqrt{x_{0}}\right\}$. To obtain the desired regularity of $V$ we will differentiate the equation (4.8) and obtain energy estimates for the resulting system of differential equations. We begin by proving

Lemma 4.1. For $T>0$, let $\Omega=[0, T] \times[0, T] \times \partial X$. Let $V\left(x^{\prime}, t^{\prime}, y\right)$ be a $N \times 1$ vector, smooth in $x^{\prime}>0$, $t^{\prime}>0$, satisfying the system

$$
\begin{gathered}
\left(\frac{\partial}{\partial x^{\prime}} \frac{\partial}{\partial t^{\prime}}+x^{\prime} t^{\prime} \Delta_{h}\right) V+x^{\prime} t^{\prime} B\left(x^{\prime}, t^{\prime}, y, \frac{\partial}{\partial y}\right) V+C\left(x^{\prime}, t^{\prime}, y\right) t^{\prime} \frac{\partial V}{\partial t^{\prime}}+D\left(x^{\prime}, t^{\prime}, y\right) x^{\prime} \frac{\partial V}{\partial x^{\prime}}+ \\
E\left(x^{\prime}, t^{\prime}, y\right) V=0 \text { in } \quad x^{\prime}>0, \quad t^{\prime}>0 \quad \text { and } \\
V\left(x^{\prime}, x^{\prime}, y\right)=f_{1}\left(x^{\prime}, y\right), \quad \frac{\partial V}{\partial x^{\prime}}\left(x^{\prime}, x^{\prime}, y\right)=f_{2}\left(x^{\prime}, y\right), \quad x^{\prime}>0,
\end{gathered}
$$

where $B\left(x^{\prime}, t^{\prime}, y, \frac{\partial}{\partial y}\right)$ is an $N \times N$ matrix of first order differential operators having derivatives in $y$ only, $C, D$ and $E$ are $N \times N$ matrices of functions. Moreover $B, C, D$ and $E$ are smooth in $\left|x^{\prime}\right|<T,\left|t^{\prime}\right|<T$, $y \in \partial X$. If the data $f_{1}$ and $f_{2}$ are such that

$$
\int_{0}^{T} \int_{\partial X}\left(x^{\prime}\left|f_{1}\right|^{2}+x^{\prime 3}\left|\nabla_{y} f_{1}\right|^{2}\right) \sqrt{h}\left(x^{\prime 2}, y\right) d x^{\prime} d y<\infty, \quad \int_{0}^{T} \int_{\partial X} x^{\prime}\left|f_{2}\right|^{2} \sqrt{h}\left(x^{\prime 2}, y\right) d x^{\prime} d y<\infty
$$



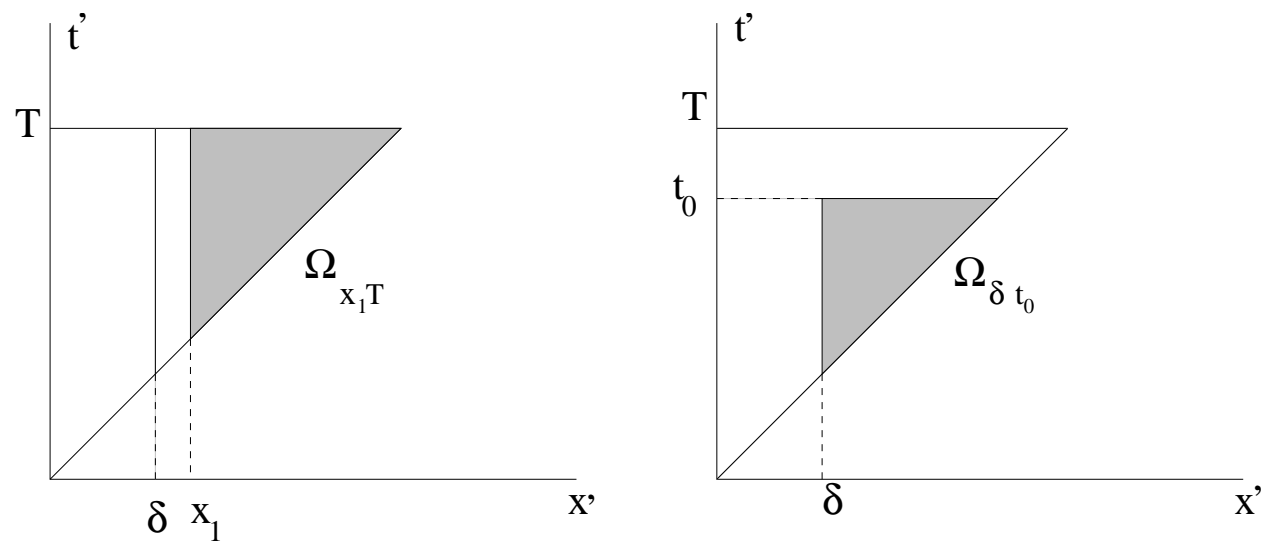

Figure 1 . The regions $\Omega_{x_{1}, T}$ and $\Omega_{\delta, t_{0}}$.

where $\left|\nabla_{y} f_{1}\right|^{2}=\sum_{j}\left|\nabla_{y} f_{1, j}\right|^{2}$, then for $T$ small

$$
\begin{gathered}
\int_{\Omega}\left[|V|^{2}+x^{\prime} t^{\prime}\left(x^{\prime}+t^{\prime}\right)\left|\nabla_{y} V\right|^{2}+x^{\prime}\left|\frac{\partial V}{\partial x^{\prime}}\right|^{2}+t^{\prime}\left|\frac{\partial V}{\partial t^{\prime}}\right|^{2}\right] \sqrt{h}\left(x^{\prime} t^{\prime}, y\right) d y d x^{\prime} d t^{\prime} \leq \\
C(T) \int_{0}^{T} \int_{\partial X}\left(x^{\prime}\left|f_{1}\right|^{2}+x^{\prime}\left|f_{2}\right|^{2}+x^{\prime 3}\left|\nabla_{y} f_{1}\right|^{2}\right)\left(x^{\prime}, y\right) \sqrt{h}\left(x^{\prime 2}, y\right) d y d x^{\prime},
\end{gathered}
$$

where $\left|\nabla_{y} V\right|^{2}=\sum_{j}\left|\nabla_{y} V_{j}\right|^{2}$

Proof. We begin by multiplying the system (4.10) by $x^{\prime} \frac{\partial V}{\partial x^{\prime}}-t^{\prime} \frac{\partial V}{\partial t^{\prime}}$. Notice that the operator $x^{\prime} \frac{\partial}{\partial x^{\prime}}-t^{\prime} \frac{\partial}{\partial t^{\prime}}$ is $\frac{\partial}{\partial t}$ written in these coordinates. We obtain

$$
\begin{gathered}
0=\frac{1}{2 \sqrt{h}\left(x^{\prime} t^{\prime}, y\right)} \frac{\partial}{\partial t^{\prime}}\left[\left(x^{\prime}\left|\frac{\partial V}{\partial x^{\prime}}\right|^{2}+t^{\prime 2} x^{\prime}\left|\nabla_{y} V\right|^{2}\right) \sqrt{h}\left(x^{\prime} t^{\prime}, y\right)\right]- \\
\frac{1}{2 \sqrt{h}\left(x^{\prime} t^{\prime}, y\right)} \frac{\partial}{\partial x^{\prime}}\left[\left(t^{\prime}\left|\frac{\partial V}{\partial t^{\prime}}\right|^{2}+t^{\prime} x^{\prime 2}\left|\nabla_{y} V\right|^{2}\right) \sqrt{h}\left(x^{\prime} t^{\prime}, y\right)\right]+ \\
+\sum_{k} x^{\prime} t^{\prime} \delta_{h}\left(\left(x^{\prime} \frac{\partial V_{k}}{\partial x^{\prime}}-t^{\prime} \frac{\partial V_{k}}{\partial t^{\prime}}\right) d_{h} V_{k}\right)+Q\left(V, x^{\prime} \frac{\partial V}{\partial x^{\prime}}, t^{\prime} \frac{\partial V}{\partial t^{\prime}}, x^{\prime} t^{\prime} \frac{\partial V}{\partial y_{j}}\right) .
\end{gathered}
$$

Here $\delta_{h}$ is the divergence operator in the $y$ variable, dual to $d_{h}$ with respect to the metric $h\left(x^{\prime} t^{\prime}, y\right)$, and $Q$ is a quadratic form with smooth coefficients.

Let

$$
\begin{gathered}
\Omega_{\delta}=[\delta, T] \times[\delta, T] \times \partial X, \quad \Omega_{\delta}^{+}=\left\{\left(x^{\prime}, t^{\prime}, y\right) \in \Omega_{\delta} ; \quad t^{\prime} \geq x^{\prime}\right\}, \quad \text { and } \\
\Omega_{a, b}=\left\{\left(x^{\prime}, t^{\prime}, y\right) \in \Omega, \quad a \leq x^{\prime} \leq t^{\prime} \leq b\right\}
\end{gathered}
$$

see figure 1 
Integrating (4.13) in $\Omega_{x_{1}, T}$, using the compactness of $\partial X$, the divergence theorem, and that the part of the first term in (4.13) which is inside the brackets is positive, we have

$$
\begin{gathered}
\frac{1}{2} \int_{x_{1}}^{T} \int_{\partial X}\left(t^{\prime}\left|\frac{\partial V}{\partial t^{\prime}}\right|^{2}+t^{\prime} x^{\prime 2}\left|\nabla_{y} V\right|^{2}\right)\left(x_{1}, t^{\prime}, y\right) \sqrt{h}\left(x_{1} t^{\prime}, y\right) d y d t^{\prime}+\int_{\Omega_{x_{1}, T}} Q \sqrt{h}\left(x^{\prime} t^{\prime}, y\right) d y d x^{\prime} d t^{\prime} \\
\leq \frac{1}{2} \int_{x_{1}}^{T} \int_{\partial X}\left(2 x^{\prime}\left|\frac{\partial V}{\partial x^{\prime}}\right|^{2}+2 x^{\prime 3}\left|\nabla_{y} V\right|^{2}\right)\left(x^{\prime}, x^{\prime}, y\right) \sqrt{h}\left(x^{\prime 2}, y\right) d y d x^{\prime} .
\end{gathered}
$$

Proceeding similarly in the region $\Omega_{\delta, t_{0}}$ gives

$$
\begin{gathered}
\frac{1}{2} \int_{\delta}^{t_{0}} \int_{\partial X}\left(x^{\prime}\left|\frac{\partial V}{\partial x^{\prime}}\right|^{2}+x^{\prime} t^{\prime 2}\left|\nabla_{y} V\right|^{2}\right)\left(x^{\prime}, t_{0}, y\right) \sqrt{h}\left(x^{\prime} t_{0}, y\right) d y d t^{\prime}+\int_{\Omega_{\delta, t_{0}}} Q \sqrt{h}\left(x^{\prime} t^{\prime}, y\right) d y d x^{\prime} d t^{\prime} \\
\leq \frac{1}{2} \int_{\delta}^{t_{0}} \int_{\partial X}\left(2 x^{\prime}\left|\frac{\partial V}{\partial x^{\prime}}\right|^{2}+2 x^{\prime 3}\left|\nabla_{y} V\right|^{2}\right)\left(x^{\prime}, x^{\prime}, y\right) \sqrt{h}\left(x^{\prime 2}, y\right) d y d x^{\prime} .
\end{gathered}
$$

Next we integrate (4.14) in $x_{1} \in[\delta, T]$ and (4.15] in $t_{0} \in[\delta, T]$ and add the results to get

$$
\begin{gathered}
\frac{1}{2} \int_{\Omega_{\delta}^{+}}\left(x^{\prime} t^{\prime}\left(t^{\prime}+x^{\prime}\right)\left|\nabla_{y} V\right|^{2}+x^{\prime}\left|\frac{\partial V}{\partial x^{\prime}}\right|^{2}+t^{\prime}\left|\frac{\partial V}{\partial t^{\prime}}\right|^{2}\right)\left(x^{\prime}, t^{\prime}, y\right) \sqrt{h}\left(x^{\prime} t^{\prime}, y\right) d y d x^{\prime} d t^{\prime}+ \\
\int_{\delta}^{T} \int_{\Omega_{x_{1}, T}} Q \sqrt{h}\left(x^{\prime} t^{\prime}, y\right) d y d x^{\prime} d t^{\prime} d x_{1}+\int_{\delta}^{T} \int_{\Omega_{\delta, t_{0}}} Q \sqrt{h}\left(x^{\prime} t^{\prime}, y\right) d y d x^{\prime} d t^{\prime} d t_{0} \leq \\
(T-\delta) \int_{\delta}^{T} \int_{\partial X}\left(x^{\prime}\left|\frac{\partial V}{\partial x^{\prime}}\right|^{2}+{x^{\prime}}^{3}\left|\nabla_{y} V\right|^{2}\right)\left(x^{\prime}, x^{\prime}, y\right) \sqrt{h}\left(x^{\prime 2}, y\right) d y d x^{\prime}
\end{gathered}
$$

With the exception of terms of $Q$ containing products involving $V_{j}$, all the others in the two middle terms of (4.16) are trivially bounded by the terms in the first integral. To analyze the terms containing $V_{j}$, we will bound the integral of $|V|^{2}$ in $\Omega_{\delta}^{+}$. So we write for $t^{\prime}>x^{\prime}$,

$$
V_{j}\left(x^{\prime}, t^{\prime}, y\right)=V_{j}\left(t^{\prime}, t^{\prime}, y\right)-\int_{x^{\prime}}^{t^{\prime}} \frac{\partial V_{j}}{\partial s}\left(s, t^{\prime}, y\right) d s .
$$

The Cauchy-Schwartz inequality then gives

$$
\left|V_{j}\left(x^{\prime}, t^{\prime}, y\right)\right|^{2} \leq 2\left|V_{j}\left(t^{\prime}, t^{\prime}, y\right)\right|^{2}+2\left(t^{\prime}-x^{\prime}\right) \int_{x^{\prime}}^{t^{\prime}}\left|\frac{\partial V_{j}}{\partial s}(s, t, y)\right|^{2} d s .
$$

Hence, as $\sqrt{h}$ is bounded from above and below, there exists $C>0$ such that

$$
\begin{gathered}
\int_{\Omega_{\delta}^{+}}\left|V_{j}\left(x^{\prime}, t^{\prime}, y\right)\right|^{2} \sqrt{h}\left(x^{\prime} t^{\prime}, y\right) d y d x^{\prime} d t^{\prime}=\int_{\delta}^{T} \int_{\delta}^{t^{\prime}} \int_{\partial X}\left|V_{j}\left(x^{\prime}, t^{\prime}, y\right)\right|^{2} \sqrt{h}\left(x^{\prime} t^{\prime}, y\right) d y d x^{\prime} d t^{\prime} \leq \\
C\left(\int_{\delta}^{T} \int_{\partial X} t^{\prime}\left|V_{j}\left(t^{\prime}, t^{\prime}, y\right)\right|^{2} \sqrt{h}\left(t^{\prime 2}, y\right) d y d t^{\prime}+\int_{\delta}^{T} \int_{\delta}^{t^{\prime}} \int_{\partial X} t^{\prime} \int_{x^{\prime}}^{t^{\prime}}\left|\frac{\partial V_{j}}{\partial s}\left(s, t^{\prime}, y\right)\right|^{2} \sqrt{h}\left(s t^{\prime}, y\right) d s d y d x^{\prime} d t^{\prime}\right) .
\end{gathered}
$$

Switching the order of integration of $y, x^{\prime}$ and $s$ in the last integral, and using that $t^{\prime}<T$, gives

$$
\begin{gathered}
\int_{\Omega_{\delta}^{+}}\left|V\left(x^{\prime}, t^{\prime}, y\right)\right|^{2} \sqrt{h}\left(x^{\prime} t^{\prime}, y\right) d y d x^{\prime} d t^{\prime} \leq C \int_{\delta}^{T} \int_{\partial X} t^{\prime}\left|V_{j}\left(t^{\prime}, t^{\prime}, y\right)\right|^{2} \sqrt{h}\left(t^{\prime 2}, y\right) d y d t^{\prime}+ \\
C T \int_{\Omega_{\delta}^{+}} s\left|\frac{\partial V_{j}}{\partial s}(s, t, y)\right|^{2} \sqrt{h}\left(s t^{\prime}, y\right) d y d s d t^{\prime} .
\end{gathered}
$$


Hence

$$
\begin{gathered}
\int_{\delta}^{T} \int_{\Omega_{x_{1}, T}}\left|Q \sqrt{h}\left(x^{\prime} t^{\prime}, y\right)\right| d y d x^{\prime} d t^{\prime} d x_{1}+\int_{\delta}^{T} \int_{\Omega_{\delta, t_{0}}}\left|Q \sqrt{h}\left(x^{\prime} t^{\prime}, y\right)\right| d y d x^{\prime} d t^{\prime} d t_{0} \leq \\
C(T-\delta) \int_{\Omega_{\delta}^{+}}\left[x^{\prime}\left|\frac{\partial V}{\partial x^{\prime}}\right|^{2}+t^{\prime}\left|\frac{\partial V}{\partial t^{\prime}}\right|^{2}+\left(x^{\prime} t^{\prime}\right)^{2}\left|\nabla_{y} V_{j}\right|^{2}\right] \sqrt{h}\left(x^{\prime} t^{\prime}, y\right) d y d x^{\prime} d t^{\prime}+ \\
C(T-\delta) \int_{\delta}^{T} \int_{\partial X} x^{\prime}|V|^{2}\left(x^{\prime}, x^{\prime}, y\right) \sqrt{h}\left(x^{\prime 2}, y\right) d y d x^{\prime} .
\end{gathered}
$$

After taking the limit as $\delta \rightarrow 0$, we find that there exists $K>0$ such that, for small $T$

$$
\begin{gathered}
\int_{\Omega^{+}}\left(x^{\prime} t^{\prime}\left(t^{\prime}+x^{\prime}\right)\left|\nabla_{y} V\right|^{2}+x^{\prime}\left|\frac{\partial V}{\partial x^{\prime}}\right|^{2}+t^{\prime}\left|\frac{\partial V}{\partial t^{\prime}}\right|^{2}\right) \sqrt{h}\left(x^{\prime} t^{\prime}, y\right) d y d x^{\prime} d t^{\prime} \leq \\
K T \int_{0}^{T} \int_{\partial X}\left(x^{\prime}|V|^{2}+x^{\prime}\left|\frac{\partial V}{\partial x^{\prime}}\right|^{2}+x^{\prime 3}\left|\nabla_{y} V\right|^{2}\right)\left(x^{\prime}, x^{\prime}, y\right) \sqrt{h}\left(x^{\prime 2}, y\right) d y d x^{\prime} .
\end{gathered}
$$

This does not quite give (4.12), as the term in $|V|^{2}$ is not yet included on the left hand side of the inequality. However, taking $\delta \rightarrow 0$ in (4.18), substituting it in (4.19), and choosing $T$ small enough, we obtain

$$
\begin{gathered}
\int_{\Omega^{+}}\left(|V|^{2}+x^{\prime} t^{\prime}\left(t^{\prime}+x^{\prime}\right)\left|\nabla_{y} V\right|^{2}+x^{\prime}\left|\frac{\partial V}{\partial x^{\prime}}\right|^{2}+t^{\prime}\left|\frac{\partial V}{\partial t^{\prime}}\right|^{2}\right) \sqrt{h}\left(x^{\prime} t^{\prime}, y\right) d y d x^{\prime} d t^{\prime} \leq \\
K \int_{0}^{T} \int_{\partial X}\left(x^{\prime}|V|^{2}+x^{\prime}\left|\frac{\partial V}{\partial x^{\prime}}\right|^{2}+x^{\prime 3}\left|\nabla_{y} V\right|^{2}\right)\left(x^{\prime}, x^{\prime}, y\right) \sqrt{h}\left(x^{\prime 2}, y\right) d y d x^{\prime}
\end{gathered}
$$

As the operator in (4.10) and the estimate (4.20) remain of the same type after switching $x^{\prime}$ and $t^{\prime}$, this estimate also holds in the region below the diagonal. This ends the proof of the Lemma.

Now we apply the Lemma 4.1 to prove Theorem 2.1] The goal is to prove that for $f(x, y)$ smooth and compactly supported, the solution to (4.2) is smooth up to $\{x=0\}$. We know from (4.6) that the change of variables (4.7) are smooth on the support of $v$, and we work in coordinates $\left(x^{\prime}, t^{\prime}\right)$. We will show that $V\left(x^{\prime}, t^{\prime}, y\right)$ is smooth up to $\left\{x^{\prime}=0\right\} \cup\left\{t^{\prime}=0\right\}$.

We first apply Lemma 4.1 to the special case of equation (4.8), noticing that in this case $V$ is a single function instead of a vector. The data on the surface $\left\{x^{\prime}=t^{\prime}\right\}$ is given by (4.8), so the integral on the right hand side of (4.12) is equal to

$$
\int_{0}^{T} \int_{\partial X} 2 x^{\prime}\left|x^{\prime-n-1} f\left(x^{\prime 2}, y\right)\right|^{2} \sqrt{h}\left(x^{\prime 2}, y\right) d y d x^{\prime}=\int_{0}^{T^{2}} \int_{\partial X}|f(x, y)|^{2} \sqrt{h}(x, y) \frac{d y d x^{\prime}}{x^{n+1}} \leq\|f\|_{L^{2}(X)}^{2} .
$$

Thus the right hand side of (4.12) is bounded by the square of the norm of $f$ in $L^{2}\left(X, d \operatorname{vol}_{g}\right)$ and

$$
\int_{\Omega_{T}}\left[|V|^{2}+x^{\prime} t^{\prime}\left(x^{\prime}+t^{\prime}\right)\left|\nabla_{y} V\right|^{2}+x^{\prime}\left|\frac{\partial V}{\partial x^{\prime}}\right|^{2}+t^{\prime}\left|\frac{\partial V}{\partial t^{\prime}}\right|^{2}\right] \sqrt{h}\left(x^{\prime} t^{\prime}, y\right) d y d x^{\prime} d t^{\prime} \leq C\|f\|_{L^{2}(X)}^{2} .
$$

Now we want to obtain such energy estimates for the derivatives of $V$. We begin by analyzing the derivatives of $V$ in the $y$ variables and thus we differentiate equation (4.8) with respect to $y$. We get a system of equations of the form

$$
\mathcal{Q} \mathcal{V}=0
$$


where $\mathcal{V}$ is a $(n+1) \times 1$ vector whose transpose is

$$
\mathcal{V}^{T}=\left(V, \frac{\partial V}{\partial y_{1}}, \ldots ., \frac{\partial V}{\partial y_{n}}\right),
$$

and $\mathcal{Q}$ is a matrix of second order operators with principal part

$$
\mathcal{Q}_{2}=\left(\frac{\partial}{\partial x^{\prime}} \frac{\partial}{\partial t^{\prime}}+x^{\prime} t^{\prime} \Delta_{h}\right) \operatorname{Id}_{(n+1) \times(n+1)},
$$

and lower order terms as in in (4.10). So we conclude that

$$
\begin{gathered}
\int_{\Omega}\left[|\mathcal{V}|^{2}+x^{\prime} t^{\prime}\left(x^{\prime}+t^{\prime}\right)\left|\nabla_{y} \mathcal{V}\right|^{2}+x^{\prime}\left|\frac{\partial \mathcal{V}}{\partial x^{\prime}}\right|^{2}+t^{\prime}\left|\frac{\partial \mathcal{V}}{\partial t^{\prime}}\right|^{2}\right] \sqrt{h}\left(x^{\prime} t^{\prime}, y\right) d y d x^{\prime} d t^{\prime} \leq \\
C\left(\|f\|_{L^{2}(X)}^{2}+\left\|\nabla_{y} f\right\|_{L^{2}(X)}^{2}\right) .
\end{gathered}
$$

Using this argument repeatedly we conclude that

$$
\begin{gathered}
\sum_{|\alpha| \leq k}\left[\int_{\Omega}\left[\left|\left(\frac{\partial}{\partial y}\right)^{\alpha} V\right|^{2}+x^{\prime}\left|\frac{\partial}{\partial x^{\prime}}\left(\frac{\partial}{\partial y}\right)^{\alpha} V\right|^{2}+t^{\prime}\left|\frac{\partial}{\partial t^{\prime}}\left(\frac{\partial}{\partial y}\right)^{\alpha} V\right|^{2}\right] \sqrt{h}\left(x^{\prime} t^{\prime}, y\right) d y d x^{\prime} d t^{\prime}\right] \leq \\
C \sum_{|\alpha| \leq k+1}\left\|\left(\frac{\partial}{\partial y}\right)^{\alpha} f\right\|_{L^{2}(X)}^{2}, \quad k \in \mathbb{N},
\end{gathered}
$$

Next we use the equation to obtain information about the derivatives of $V$ with respect to $x^{\prime}$ and $t^{\prime}$. It is convenient to get rid of the first order terms in (4.8), and we do that by conjugating the operator with the factor $\left|h\left(x^{\prime} t^{\prime}, y\right)\right|^{-\frac{1}{4}}$. Setting

$$
W\left(x^{\prime}, t^{\prime}, y\right)=|h|^{\frac{1}{4}} V\left(x^{\prime}, t^{\prime}, y\right),
$$

then $W$ satisfies

$$
\left(\frac{\partial^{2}}{\partial x^{\prime} \partial t^{\prime}}+x^{\prime} t^{\prime} \Delta_{h}+x^{\prime} t^{\prime} B\left(x^{\prime} t^{\prime}, y, \frac{\partial}{\partial y}\right)+C\left(x^{\prime} t^{\prime}, y\right)\right) W=0,
$$

where $B$ is a first order operator and $C$ is a smooth function. Since $|h|$ is smooth and positive, $V$ and $W$ have the same regularity given by 4.21). Moreover $W$ is also supported in $\left\{x^{\prime} \geq \sqrt{x_{0}}\right\} \cup\left\{t^{\prime} \geq \sqrt{x_{0}}\right\}$.

We have shown in (4.21) that $t^{\prime \frac{1}{2}} \frac{\partial}{\partial t^{\prime}} \frac{\partial^{\alpha}}{\partial y^{\alpha}} W \in L^{2}(\Omega)$ for any $\alpha$. In particular if $\Omega^{+}=\Omega \cap\left\{t^{\prime}>x^{\prime}\right\}$, $t^{\prime \frac{1}{2}} \frac{\partial}{\partial t^{\prime}} \frac{\partial^{\alpha}}{\partial y^{\alpha}} W \in L^{2}\left(\Omega^{+}\right)$. In view of the support of $W$ we have $\frac{\partial}{\partial t^{\prime}} \frac{\partial^{\alpha}}{\partial y^{\alpha}} W \in L^{2}\left(\Omega^{+}\right)$.

We deduce from (4.21) and (4.23) that

$$
\left(\frac{\partial}{\partial y}\right)^{\alpha} W\left(x^{\prime}, t^{\prime}, y\right), \quad \frac{\partial}{\partial t^{\prime}}\left(\frac{\partial}{\partial y}\right)^{\alpha} W\left(x^{\prime}, t^{\prime}, y\right), \quad\left(\frac{\partial}{\partial y}\right)^{\alpha} \frac{\partial}{\partial t^{\prime}} \frac{\partial}{\partial x^{\prime}} W\left(x^{\prime}, t^{\prime}, y\right) \in L^{2}\left(\Omega^{+}\right) \forall \alpha \in \mathbb{N}^{n}
$$

Writing, for $t^{\prime}>x^{\prime}$,

$$
\frac{\partial}{\partial x^{\prime}}\left(\frac{\partial}{\partial y}\right)^{\alpha} W\left(x^{\prime}, t^{\prime}, y\right)=\frac{\partial}{\partial x^{\prime}}\left(\frac{\partial}{\partial y}\right)^{\alpha} W\left(x^{\prime}, x^{\prime}, y\right)+\int_{x^{\prime}}^{t^{\prime}} \frac{\partial}{\partial \mu} \frac{\partial}{\partial x^{\prime}}\left(\frac{\partial}{\partial y}\right)^{\alpha} W\left(x^{\prime}, \mu, y\right) d \mu,
$$

we see that, for $t^{\prime}>x^{\prime}$,

$$
\begin{gathered}
\left|\frac{\partial}{\partial x^{\prime}}\left(\frac{\partial}{\partial y}\right)^{\alpha} W\left(x^{\prime}, t^{\prime}, y\right)\right|^{2} \leq \\
2\left|\frac{\partial}{\partial x^{\prime}}\left(\frac{\partial}{\partial y}\right)^{\alpha} W\left(x^{\prime}, x^{\prime}, y\right)\right|^{2}+2\left(t^{\prime}-x^{\prime}\right) \int_{x^{\prime}}^{t^{\prime}}\left|\frac{\partial}{\partial \mu} \frac{\partial}{\partial x^{\prime}}\left(\frac{\partial}{\partial y}\right)^{\alpha} W\left(x^{\prime}, \mu, y\right)\right|^{2} d \mu .
\end{gathered}
$$


Integrating this in $\Omega^{+}$, using that $\frac{\partial}{\partial x^{\prime}} \frac{\partial^{\alpha}}{\partial y^{\alpha}} W\left(x^{\prime}, x^{\prime}, y\right)$ is smooth with compact support, and (4.24), we find that

$$
\frac{\partial}{\partial x^{\prime}}\left(\frac{\partial}{\partial y}\right)^{\alpha} W \in L^{2}\left(\Omega^{+}\right), \quad \alpha \in \mathbb{N}^{n}
$$

So (4.24) and (4.25) show that

$$
\left(\frac{\partial}{\partial y}\right)^{\alpha} W, \frac{\partial}{\partial x^{\prime}}\left(\frac{\partial}{\partial y}\right)^{\alpha} W \text { and } \frac{\partial}{\partial t^{\prime}}\left(\frac{\partial}{\partial y}\right)^{\alpha} W \in L^{2}\left(\Omega^{+}\right), \quad \alpha \in \mathbb{N}^{n} .
$$

Using the symmetry of $W$ with respect to the diagonal, we in fact have

$$
\left(\frac{\partial}{\partial y}\right)^{\alpha} W, \frac{\partial}{\partial x^{\prime}}\left(\frac{\partial}{\partial y}\right)^{\alpha} W \text { and } \frac{\partial}{\partial t^{\prime}}\left(\frac{\partial}{\partial y}\right)^{\alpha} W \in L^{2}(\Omega), \quad \alpha \in \mathbb{N}^{n}
$$

Differentiating (4.23) first with respect to $y$ and then with respect to $x^{\prime}$ or $t^{\prime}$ we find that

$$
\begin{aligned}
\frac{\partial^{2}}{\partial{x^{\prime}}^{2}} \frac{\partial}{\partial t^{\prime}}\left(\frac{\partial}{\partial y}\right)^{\alpha} W & =\sum_{\beta} \sum_{m=0,1} G_{\beta, m}\left(x^{\prime}, t^{\prime}, y\right) \frac{\partial^{m}}{\partial x^{\prime m}}\left(\frac{\partial}{\partial y}\right)^{\beta} W \in L^{2}\left(\Omega^{+}\right), \\
\frac{\partial^{2}}{\partial t^{\prime 2}} \frac{\partial}{\partial x^{\prime}}\left(\frac{\partial}{\partial y}\right)^{\alpha} W & =\sum_{\beta} \sum_{m=0,1} F_{\beta, m}\left(x^{\prime}, t^{\prime}, y\right) \frac{\partial^{m}}{\partial t^{\prime m}}\left(\frac{\partial}{\partial y}\right)^{\beta} W \in L^{2}\left(\Omega^{+}\right),
\end{aligned}
$$

with $F_{\beta, m}$ and $G_{\beta, m}$ smooth. Thus

$$
\frac{\partial^{2}}{\partial x^{\prime 2}} \frac{\partial}{\partial t^{\prime}}\left(\frac{\partial}{\partial y}\right)^{\alpha} W, \frac{\partial^{2}}{\partial t^{\prime 2}} \frac{\partial}{\partial x^{\prime}}\left(\frac{\partial}{\partial y}\right)^{\alpha} W \in L^{2}\left(\Omega^{+}\right)
$$

Proceeding as above, we find that

$$
\frac{\partial^{2}}{\partial x^{\prime 2}}\left(\frac{\partial}{\partial y}\right)^{\alpha} W, \quad \frac{\partial^{2}}{\partial t^{\prime 2}}\left(\frac{\partial}{\partial y}\right)^{\alpha} W, \quad \text { and } \frac{\partial}{\partial x^{\prime}} \frac{\partial}{\partial t^{\prime}}\left(\frac{\partial}{\partial y}\right)^{\alpha} W \in L^{2}\left(\Omega^{+}\right) .
$$

Using the symmetry of $W$ with respect to the diagonal we get that in fact

$$
\frac{\partial^{2}}{\partial x^{\prime 2}}\left(\frac{\partial}{\partial y}\right)^{\alpha} W, \quad \frac{\partial^{2}}{\partial t^{\prime 2}}\left(\frac{\partial}{\partial y}\right)^{\alpha} W, \quad \text { and } \quad \frac{\partial}{\partial x^{\prime}} \frac{\partial}{\partial t^{\prime}}\left(\frac{\partial}{\partial y}\right)^{\alpha} W \in L^{2}(\Omega) .
$$

This argument can be repeated for all derivatives with respect to $x^{\prime}$ and $t^{\prime}$ and we conclude that $W$, and hence $V$, is smooth in $[0, T] \times[0, T] \times \partial X$ for $T$ small. Any interval $\left[0, T^{\prime}\right]$ can be covered by small intervals in which the method above can be applied. So in fact this shows that the solution is smooth in $[0, T] \times[0, T] \times \partial X$ for any $T$.

Since $V$ is supported in $\left\{x^{\prime}>\sqrt{x_{0}}\right\} \cup\left\{t^{\prime}>\sqrt{x_{0}}\right\}$ and the change of coordinates (4.7) is smooth in this region, this shows that $v$ has a smooth extension up to $\{x=0\}$. This concludes the proof of Theorem 2.1]

\section{The Radiation Fields And The Scattering Matrix}

The spectrum of the Laplacian $\sigma(\Delta)$ was studied by Mazzeo and Mazzeo and Melrose 39, 40, 43, see also section 3 of [16] for a discussion. It consists of a finite pure point spectrum $\sigma_{\mathrm{pp}}(\Delta)$, which is the set of $L^{2}(X)$ eigenvalues, and an absolutely continuous spectrum $\sigma_{\mathrm{ac}}(\Delta)$ satisfying

$$
\sigma_{\mathrm{ac}}(\Delta)=\left[\frac{n^{2}}{4}, \infty\right), \quad \text { and } \quad \sigma_{\mathrm{pp}}(\Delta) \subset\left(0, \frac{n^{2}}{4}\right) .
$$

This gives a decomposition

$$
L^{2}(X)=L_{\mathrm{pp}}^{2}(X) \oplus L_{\mathrm{ac}}^{2}(X),
$$


where $L_{\mathrm{pp}}^{2}(X)$ is the finite dimensional space spanned by the eigenfunctions and $L_{\mathrm{ac}}^{2}(X)$ is the space of absolute continuity, which is the orthogonal complement of $L_{\mathrm{pp}}^{2}(X)$.

With this choice of the spectral parameter, $\frac{n^{2}}{4}+\lambda^{2}$, we have that if $\Im \lambda \neq 0$, then $\frac{n^{2}}{4}+\lambda^{2} \notin\left[\frac{n^{2}}{4}, \infty\right)$, while if $\Im \lambda<-\frac{n}{2}$ it follows that $\frac{n^{2}}{4}+\lambda^{2} \notin[0, \infty)$. The eigenvalues of $\Delta$ are of finite multiplicity and are described by points on the line $\Re \lambda=0$ and $-\frac{n}{2}<\Im \lambda<0$. As the Laplacian is a non-negative operator, the spectral theorem gives that the resolvent

$$
R\left(\frac{n}{2}+i \lambda\right)=\left(\Delta-\frac{n^{2}}{4}-\lambda^{2}\right)^{-1}: L^{2}(X) \longrightarrow L^{2}(X), \quad \text { provided } \Im \lambda<-\frac{n}{2} .
$$

It was shown in [43] that it can be meromorphically continued to $\mathbb{C} \backslash \frac{i}{2} \mathbb{N}$ as an operator acting on appropriate spaces.

Let

$$
H_{E}(X)=\left\{\left(f_{1}, f_{2}\right): \quad f_{1}, f_{2} \in L^{2}(X), \quad \text { and } d f_{1} \in L^{2}(X)\right\} .
$$

For $w_{0}, w_{1} \in C_{0}^{\infty}(\stackrel{\circ}{X})$, we define the energy of $w=\left(w_{0}, w_{1}\right)$ by

$$
\|w\|_{E}^{2}=\frac{1}{2} \int_{X}\left(\left|d w_{0}\right|_{g}^{2}-\frac{n^{2}}{4}\left|w_{0}\right|^{2}+\left|w_{1}\right|^{2}\right) \quad d \operatorname{vol}_{g},
$$

where $\left|d w_{0}\right|_{g}$ denotes the length of the co-vector with respect to the metric induced by $g$ on $T^{*} X$. But $\|w\|_{E}^{2}$ is only positive when $w_{0}$ is in the space of absolute continuity of $\Delta$, and only then it defines a norm. We denote

$$
\mathcal{P}_{\mathrm{ac}}: L^{2}(X) \longrightarrow L_{\mathrm{ac}}^{2}(X)
$$

the corresponding projector. Let

$$
E_{\text {ac }}(X)=\mathcal{P}_{\text {ac }}\left(H_{E}(X)\right)=\text { Range of the projector } \mathcal{P}_{\text {ac }} \text { acting on } H_{E}(X) .
$$

$E_{\text {ac }}(X)$ is a Hilbert space equipped with the norm (5.3. .

One can use integration by parts to prove that if $u(t, z)$ satisfies (2.5), then

$$
\left\|\left(u(t, \bullet), D_{t} u(t, \bullet)\right)\right\|_{E}=\left\|\left(u(0, \bullet), D_{t} u(0, \bullet)\right)\right\|_{E} .
$$

Since $W(t)$ commutes with $\mathcal{P}_{\text {ac }}$ this gives, by for example slightly modifying the proof of Proposition 2.24 of [13, that the map $W(t)$ defined by

$$
\begin{gathered}
W(t): C_{0}^{\infty}(\stackrel{\circ}{X}) \times C_{0}^{\infty}(\stackrel{\circ}{X}) \longrightarrow C_{0}^{\infty}(\stackrel{\circ}{X}) \times C_{0}^{\infty}(\stackrel{\circ}{X}) \\
W(t)\left(f_{1}, f_{2}\right)=\left(u(t, z), D_{t} u(t, z)\right), \quad t \in \mathbb{R}
\end{gathered}
$$

induces a strongly continuous group of unitary operators.

$$
W(t): E_{\mathrm{ac}}(X) \longrightarrow E_{\mathrm{ac}}(X), \quad t \in \mathbb{R} .
$$

By changing $t \leftrightarrow t-\tau$, one has that $\mathcal{R}_{ \pm}$satisfy

$$
\mathcal{R}_{ \pm} \circ(W(\tau) f)(s, y)=\mathcal{R}_{ \pm} f(s+\tau, y), \quad \tau \in \mathbb{R} .
$$

So Theorem 2.1 shows that $\mathcal{R}_{ \pm}$are "twisted" translation representations of the group $W(t)$. That is, if one sets $\widetilde{\mathcal{R}_{ \pm}}(f)(s, y)=\mathcal{R}_{ \pm} f(-s, y)$, then

$$
\widetilde{\mathcal{R}_{ \pm}}(W(\tau))=T_{\tau} \widetilde{\mathcal{R}_{ \pm}}
$$

where $T_{\tau}$ denotes right translation by $\tau$ in the $s$ variable. So $\widetilde{\mathcal{R}_{ \pm}}$are translation representers in the sense of Lax and Phillips. Moreover we will prove 
Theorem 5.1. The maps $\mathcal{R}_{ \pm}$induce isometries

$$
\mathcal{R}_{ \pm}: E_{\mathrm{ac}}(X) \longmapsto L^{2}(\mathbb{R} \times \partial X),
$$

where $L^{2}(\mathbb{R} \times \partial X)$ is defined with respect to $h_{0}$ fixed in (2.4).

We deduce from Theorem 5.1 that the scattering operator

$$
\begin{gathered}
\mathcal{S}: L^{2}(\mathbb{R} \times \partial X) \longrightarrow L^{2}(\mathbb{R} \times \partial X) \\
\mathcal{S}=\mathcal{R}_{+} \circ \mathcal{R}_{-}^{-1}
\end{gathered}
$$

is unitary in $L^{2}(\partial X \times \mathbb{R})$, and in view of (5.6), it commutes with translations. This implies that the Schwartz kernel $\mathcal{S}\left(s, y, s^{\prime}, y^{\prime}\right)$ of $\mathcal{S}$ satisfies

$$
\mathcal{S}\left(s, y, s^{\prime}, y^{\prime}\right)=\mathcal{S}\left(s-s^{\prime}, y, y^{\prime}\right),
$$

and thus is a convolution operator. The scattering matrix is defined by conjugating $\mathcal{S}$ with the partial Fourier transform in the $s$ variable

$$
\mathcal{A}=\mathcal{F S F}^{-1} \text {. }
$$

$\mathcal{A}$ is a unitary operator in $L^{2}(\partial X \times \mathbb{R})$ and, since $\mathcal{S}$ acts as a convolution in the variable $s, \mathcal{A}$ is a multiplication in the variable $\lambda$, i.e. it satisfies

$$
\mathcal{A} F(\lambda, y)=\int_{\partial X} \mathcal{A}\left(\lambda, y, y^{\prime}\right) F\left(\lambda, y^{\prime}\right) d \operatorname{vol}_{h_{0}}\left(y^{\prime}\right)
$$

We will prove that the stationary and dynamical definitions of the scattering matrix are equivalent:

Theorem 5.2. With $x$ given by (2.4) and $\lambda \neq 0$, the Schwartz kernel of the map $A(\lambda)$ defined by (2.3), is equal to $\mathcal{A}\left(\lambda, y, y^{\prime}\right)$, defined in (5.8).

To prove Theorem 5.1 and Theorem 5.2 we will use the connection between the wave equation, the resolvent, and the Eisenstein function from [28, 43].

\section{The Radiation Fields and the Eisenstein Function}

The following is an important fact concerning the behavior of the solution to the wave equation:

Proposition 6.1. Let $f=\left(f_{1}, f_{2}\right) \in H_{E}(X)$ and let $u(t, z)$ be the solution to (2.5) with initial data $f$. There exists $C=C(f)>0$ such that

$$
\int_{X}\left|\frac{\partial u(t, z)}{\partial t}\right|^{2} d \operatorname{vol}_{g}(z) \leq C e^{\frac{n}{2} t}, \quad t>0
$$

Proof. In view of (5.1) we can write

$$
f_{j}(z)=\sum c_{j, k} \phi_{k}(z)+g_{j}(z)
$$

where $\phi_{k}$ is an eigenfunction associated with an eigenvalue $\sigma_{k}=\frac{n^{2}}{4}+\lambda_{k}^{2} \in\left(0, \frac{n^{2}}{4}\right)$ and $g_{j}$ is the projection of $f_{j}$ onto $L_{\mathrm{ac}}^{2}(X)$.

We then have that

$$
u(t, z)=u_{\mathrm{ac}}(t, z)+\sum_{k}\left(c_{1, k} \cos \left(\lambda_{k} t\right)+c_{2, k} \frac{1}{\lambda_{k}} \sin \left(\lambda_{k} t\right)\right) \phi_{k}(z)
$$

where $u_{a c}$ is the solution to (2.5) with data $g=\left(g_{1}, g_{2}\right)$. 
As

$$
u_{\mathrm{ac}}(t, z)=\cos t \sqrt{\Delta-\frac{n^{2}}{4}} g_{1}+\left(\Delta-\frac{n^{2}}{4}\right)^{-\frac{1}{2}} \sin t \sqrt{\Delta-\frac{n^{2}}{4}} g_{2},
$$

it follows that

$$
\mathcal{P}_{\mathrm{ac}} u(t, z)=\cos t \sqrt{\Delta-\frac{n^{2}}{4}} \mathcal{P}_{\mathrm{ac}} f_{1}+\left(\Delta-\frac{n^{2}}{4}\right)^{-\frac{1}{2}} \sin t \sqrt{\Delta-\frac{n^{2}}{4}} \mathcal{P}_{\mathrm{ac}} f_{2}=u_{\mathrm{ac}}(t, z) .
$$

In this case the energy

$$
\begin{gathered}
\int_{X}\left(\left|\frac{\partial u_{\mathrm{ac}}(t, z)}{\partial t}\right|^{2}+\left|\nabla_{y} u_{\mathrm{ac}}(t, z)\right|^{2}-\frac{n^{2}}{4}\left|u_{\mathrm{ac}}(t, z)\right|^{2}\right) d \operatorname{vol}_{g}(z)= \\
\int_{X}\left(\left|g_{2}(z)\right|^{2}+\left|\nabla_{y} g_{1}(z)\right|^{2}-\frac{n^{2}}{4}\left|g_{1}(z)\right|^{2}\right) d \operatorname{vol}_{g}(z)=E_{0}>0,
\end{gathered}
$$

and in particular this gives

$$
\int_{X}\left|\frac{\partial u_{\mathrm{ac}}(t, z)}{\partial t}\right|^{2} d \operatorname{vol}_{g}(z) \leq C .
$$

Since $0>\Im \lambda_{k}>-\frac{n}{2}$, the other part of the solution obviously satisfies (6.1) and this proves the proposition.

Next we present an elementary and useful Lemma. The proof we gave of this result in the first version of this paper was incorrect. One of the referees pointed out the mistake and kindly provided us with the proof we present.

Lemma 6.2. Let $\mathcal{H}$ be an infinite dimensional Hilbert space, let $H$ be a subspace of $\mathcal{H}$ of finite dimension and let $H^{\perp}$ be the orthogonal to $H$. If $E \subset \mathcal{H}$ is a dense subspace of $\mathcal{H}$, then $E \cap H^{\perp} \neq \emptyset$, and $E \cap H^{\perp}$ is dense in $H^{\perp}$.

Proof. We begin with the case $\operatorname{dim} H=1$ and we will prove that $\left(E \cap H^{\perp}\right)^{\perp}=H$. It is easy to see that $H \subset\left(E \cap H^{\perp}\right)^{\perp}$. To prove that $\left(E \cap H^{\perp}\right)^{\perp} \subset H$ we let $H=\operatorname{span}\{\phi\}, \phi \neq 0$. As $E$ is dense, pick $f_{1}, f_{2} \in E$ such that $\left\langle f_{j}, \phi\right\rangle \neq 0, j=1,2$. Then

$$
f=f_{2}-\frac{\left\langle f_{2}, \phi\right\rangle}{\left\langle f_{1}, \phi\right\rangle} f_{1} \in E \cap H^{\perp} .
$$

Thus, if $u \in\left(E \cap H^{\perp}\right)^{\perp}$, then $\langle u, f\rangle=0$. But this can be rewritten as

$$
\left\langle f_{2}, u-\frac{\left\langle f_{1}, u\right\rangle}{\left\langle f_{1}, \phi\right\rangle} \phi\right\rangle=0, \text { for all } f_{2} \quad \text { with }\left\langle f_{2}, \phi\right\rangle \neq 0
$$

Since $E$ is dense, this in fact holds for every $f_{2}$ and thus $u=\frac{\left\langle f_{1}, u\right\rangle}{\left\langle f_{1}, \phi\right\rangle} \phi$. Therefore $\left(E \cap H^{\perp}\right)^{\perp} \subset H$.

The general case follows by induction. Suppose the result is true for $\operatorname{dim} H=N-1$ and let $\operatorname{dim} H=N$. Then

$H=H_{N-1} \oplus D, \quad H_{N-1}=\operatorname{span}\left\{\phi_{1}, \ldots, \phi_{N-1}\right\} \quad$ and $\quad D=\operatorname{span}\left\{\phi_{N}\right\}, \quad\left\langle\phi_{i}, \phi_{j}\right\rangle=0, \quad 1 \leq i, j \leq N$.

As proved above, $E_{1}=E \cap D^{\perp}$ is dense in $\mathcal{H}_{1}=D^{\perp}$. By assumption the result holds in dimension $N-1$, so $E_{1} \cap H_{N-1}^{\perp}$ is dense in the orthogonal to $H_{N-1}$ in $D^{\perp}$, which is the orthogonal to $H_{N}$ in $\mathcal{H}$.

As $E_{1} \cap H_{N-1}^{\perp}=E \cap H_{N}^{\perp}, E \cap H_{N}^{\perp}$ is dense in $H_{N}^{\perp}$ in $\mathcal{H}$.

The most important consequence of this is 
Corollary 6.3. For $L_{\mathrm{ac}}^{2}(X)$ defined as above, $C_{0}^{\infty}(\stackrel{\circ}{X}) \cap L_{\mathrm{ac}}^{2}(X)$ is dense in $L_{\mathrm{ac}}^{2}(X)$.

Now we are ready to prove the first mapping property of $\mathcal{R}_{+}$.

Proposition 6.4. Let $u$ be the solution to (2.5) with data $f=\left(f_{1}, f_{2}\right) \in C_{0}^{\infty}(\stackrel{\circ}{X}) \cap E_{\mathrm{ac}}(X)$. Then $\mathcal{R}_{+} f(s, y) \in L^{2}(\mathbb{R} \times \partial X)$ and

$$
\left\|\mathcal{R}_{+} f\right\|_{L^{2}(\mathbb{R} \times \partial X)} \leq 2|| f \|_{E} .
$$

Proof. We modify the proof of Lemma 2.6 of [13. Let $u$ be the solution of (2.5) with initial data equal to $f$. For $t, T>0$ fixed, consider the integral

$$
E_{T, t}=\int_{\{z=(x, y) \in X ; t+\log x<T\}}\left|\frac{\partial u}{\partial t}(x, y, t)\right|^{2} d \operatorname{vol}_{g} .
$$

For $t+\log x<T$, it follows that $x \rightarrow 0$ as $t \rightarrow \infty$. So, for $t$ large, we may work in local coordinates where (2.4) holds. First we prove that

$$
\lim _{t \rightarrow \infty} E_{T, t}=\int_{-\infty}^{T} \int_{\partial X}\left|\mathcal{R}_{+} f\right|^{2} \quad d \operatorname{vol}_{h_{0}} d s, \quad \forall T \in \mathbb{R} .
$$

To see this we set

$$
u(t, x, y)=x^{\frac{n}{2}} v(t+\log x, x, y),
$$

and since $s=t+\log x$, we have $\frac{\partial u}{\partial t}=x^{\frac{n}{2}} \frac{\partial v}{\partial s}$. As $d s=\frac{d x}{x}$, it follows that $d \operatorname{vol}_{g}=\sqrt{|h|}(x, y) \frac{d x d y}{x^{n+1}}=$ $\sqrt{|h|}\left(e^{(s-t)}, y\right) e^{n(t-s)} d s d y$. Since the initial data is compactly supported, there exists $s_{0}$ such that $v=0$ for $s<s_{0}$. Thus

$$
E_{T, t}=\int_{s_{0}}^{T} \int_{\partial X}\left|\frac{\partial v}{\partial s}\right|^{2}\left(e^{s-t}, y, s\right) \sqrt{|h|\left(e^{(s-t)}, y\right)} d y d s .
$$

From Theorem 2.1 the convergence is uniform and thus we obtain

$$
\lim _{t \rightarrow \infty} E_{T, t}=\int_{-\infty}^{T} \int_{\partial X}\left|\frac{\partial v}{\partial s}(0, s, y)\right|^{2} \sqrt{|h|(0, y)} d s d y=\int_{-\infty}^{T} \int_{\partial X}\left|\mathcal{R}_{+} f(s, y)\right|^{2} \quad d \operatorname{vol}_{h_{0}} d s<\infty \quad \forall T \in \mathbb{R} .
$$

For any $\left(f_{1}, 0\right) \in E_{\mathrm{ac}}(X), \int_{X}\left(\left|d f_{1}\right|_{g}^{2}-\frac{n^{2}}{4}\left|f_{1}\right|^{2}\right) d \operatorname{vol}_{g}>0$. Thus the result follows by conservation of energy.

In view of Corollary 6.3 this can be restated as

Corollary 6.5. The maps $\mathcal{R}_{ \pm}$defined in (2.6) and (2.7) extend from $\left(C_{0}^{\infty}(\stackrel{\circ}{X}) \times C_{0}^{\infty}(\stackrel{\circ}{X})\right) \cap E_{\mathrm{ac}}(X)$ by continuity to maps

$$
\mathcal{R}_{ \pm}: E_{\mathrm{ac}}(X) \longrightarrow L^{2}(\mathbb{R} \times \partial X) .
$$

The estimate (6.1) shows that we can take partial Fourier-Laplace transform with respect to $t$ of the forward fundamental solution of the wave equation $U\left(t, z, z^{\prime}\right)$ and thus, for $\Im \lambda<-\frac{n}{2}$, we denote

$$
R\left(\frac{n}{2}+i \lambda\right)=\left(\Delta-\lambda^{2}-\frac{n^{2}}{4}\right)^{-1}=\int_{\mathbb{R}} U\left(t, z, z^{\prime}\right) e^{-i \lambda t} d t
$$

It is easy to see that if $u(t, z)$ satisfies (2.5) then $V(t, z)=H(t) u(t, z)$ satisfies

$$
\begin{gathered}
\left(D_{t}^{2}-\Delta+\frac{n^{2}}{4}\right) V(t, z)=i f_{2}(z) \delta(t)+i f_{1}(z) D_{t} \delta(t), \text { on } \mathbb{R} \times \stackrel{\circ}{X} \\
V(t, z)=0 \text { for } t<0,
\end{gathered}
$$


and $\lim _{x \rightarrow 0} x^{-\frac{n}{2}} D_{s} V(s-\log x, x, y)=\lim _{x \rightarrow 0} x^{-\frac{n}{2}} D_{s} u(s-\log x, x, y)$.

So,

$$
R\left(\frac{n}{2}+i \lambda\right)\left(i f_{2}+i \lambda f_{1}\right)=\int_{\mathbb{R}} V(t, z) e^{-i \lambda t} d t \text {, with } f=\left(f_{1}, f_{2}\right) \in C_{0}^{\infty}(X) \text { and } \quad \Im \lambda<-\frac{n}{2} .
$$

In fact, if $f=\left(f_{1}, f_{2}\right) \in C_{0}^{\infty}(X) \cap E_{\text {ac }}(X)$, conservation of (positive) energy gives that (6.3) holds for $\Im \lambda \leq 0$. Using local coordinates $z=(x, y)$ near $\partial X$, we are interested in $\lim _{x \downarrow 0} x^{-\frac{n}{2}} D_{s} V(s-\log x, x, y)$. From (6.3) we obtain, for $\Im \lambda<-\frac{n}{2}$,

$$
\begin{gathered}
\int_{\mathbb{R}} x^{-\frac{n}{2}} D_{s} V(s-\log x, y) e^{-i \lambda s} d s=\int_{\mathbb{R}} x^{-\frac{n}{2}} D_{t} V(t, x, y) e^{-i \lambda t-i \lambda \log x} d t= \\
x^{-\frac{n}{2}-i \lambda} \lambda R\left(\frac{n}{2}+i \lambda\right)\left(i f_{2}+i \lambda f_{1}\right)(x, y) .
\end{gathered}
$$

Next we study the behavior of the Schwartz kernel

$$
x^{-\frac{n}{2}-i \lambda} R\left(\frac{n}{2}+i \lambda, z, z^{\prime}\right), \quad z=(x, y), \quad z^{\prime}=\left(x^{\prime}, y^{\prime}\right), \quad \Im \lambda<-\frac{n}{2}, \text { as } x \downarrow 0 .
$$

Mazzeo and Melrose show in [43] that $R\left(\frac{n}{2}+i \lambda\right)$ has a meromorphic continuation from $\Im \lambda<-\frac{n}{2}$ to $\mathbb{C} \backslash \frac{i}{2} \mathbb{N}$. We briefly recall their construction and use it to study (6.5).

Locally, in the interior of $X \times X$, and for $\Im \lambda<<0, R\left(\frac{n}{2}+i \lambda\right)$ is pseudo-differential operator, so its kernel is singular at the diagonal

$$
D=\left\{\left(x, y, x^{\prime}, y^{\prime}\right) \in X \times X ; x=x^{\prime}, y=y^{\prime}\right\} .
$$

To understand the behavior of the kernel of $R\left(\frac{n}{2}+i \lambda\right)$ up to

$$
D_{\partial X}=D \cap(\partial X \times \partial X),
$$

and for other values of $\lambda$, Mazzeo and Melrose blow-up the intersection $D_{\partial X}$. This can be done in an invariant way, but in local coordinates this can be seen as introduction of polar coordinates around $D_{\partial X}$. Taking coordinates $(x, y)$ and $\left(x^{\prime}, y^{\prime}\right)$ in a product decomposition of each copy of $X$ near $\partial X$, the "polar coordinates' are then given by

$$
R=\left[x^{2}+x^{\prime 2}+\left|y-y^{\prime}\right|^{2}\right]^{\frac{1}{2}}, \quad \rho=\frac{x}{R}, \quad \rho^{\prime}=\frac{x^{\prime}}{R} \omega=\frac{y-y^{\prime}}{R}
$$

A function is smooth in the space $X \times_{0} X$ if it is smooth in $\left(R, \rho, \rho^{\prime}, y, \omega\right)$ about $D_{\partial X}$. As a set, $X \times_{0} X$ is $X \times X$ with $D_{\partial X}$ replaced by the interior pointing portion of its normal bundle. Let

$$
\beta: X \times_{0} X \longrightarrow X \times X
$$

denote the blow-down map.

The function $R$ is a defining function for a new face, which is called the front face, $f f$. This is the lift of $D_{\partial X}=D \cap(\partial X \times \partial X)$. The functions $\rho$ and $\rho^{\prime}$ are then defining functions for the other two boundary faces which are called the top face $\mathcal{T}$, and bottom face $\mathcal{B}$, respectively, i.e.

$$
f f=\{R=0\}, \mathcal{B}=\left\{\rho^{\prime}=0\right\}, \mathcal{T}=\{\rho=0\} .
$$

See Figure 2] which is taken from section 3 of [4]. In $X \times_{0} X$ the lift of the diagonal of $X \times X$ only meets the boundary $f f$ and is disjoint from the other two boundary faces.

The main result from [4] needed here is that the lift of the kernel of the resolvent satisfies

$$
\beta^{*} R\left(\frac{n}{2}+i \lambda\right)=R_{1}\left(\frac{n}{2}+i \lambda\right)+R_{2}\left(\frac{n}{2}+i \lambda\right),
$$



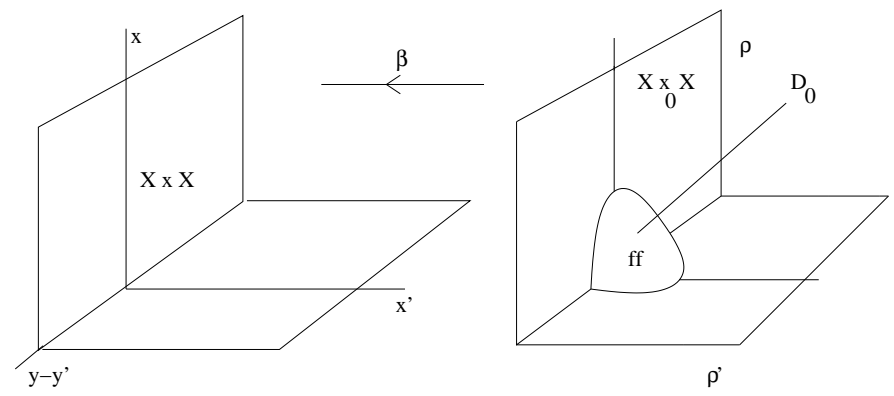

Figure 2. The blown up manifold $X \times_{0} X$.

where $R_{1}$ is conormal of order -2 to the lifted diagonal, $D_{0}$, and smooth up to the front face, and, most importantly for our purposes, vanishes to infinite order at the top and bottom faces. The second part, $R_{2}$, is of the form

$$
R_{2}=\rho^{\frac{n}{2}+i \lambda} \rho^{\prime \frac{n}{2}+i \lambda} F\left(\frac{n}{2}+i \lambda, \bullet\right),
$$

where $F\left(\frac{n}{2}+i \lambda, \bullet\right) \in C^{\infty}\left(X \times_{0} X\right)$ is meromorphic in $\lambda \in \mathbb{C} \backslash \frac{i}{2} \mathbb{N}$, and holomorphic and in $\lambda \in \Im \lambda<-\frac{n}{2}$.

Now we use the construction above to verify that $\left.x^{\prime-\frac{n}{2}-i \lambda} R\left(\frac{n}{2}+i \lambda, z, z^{\prime}\right)\right|_{\{x=0\}}$ is well defined. This is carried out in Proposition 4.1 of [28], and we briefly describe it.

We first look at the lift of $x^{-\frac{n}{2}-i \lambda} R\left(\frac{n}{2}+i \lambda, z, z^{\prime}\right)$ under the blow-down map $\beta$. Recalling that $x=R \rho$, we deduce from (6.7) and (6.8), and the fact that $R_{1}\left(\frac{n}{2}+i \lambda\right)$ vanishes to infinite order at the top and bottom faces, that

$$
\begin{gathered}
\left.\beta^{*}\left(x^{-\frac{n}{2}-i \lambda} R\left(\frac{n}{2}+i \lambda, z, z^{\prime}\right)\right)\right|_{\rho=0}=\left.(R \rho)^{-\frac{n}{2}-i \lambda}\left(R_{1}\left(\frac{n}{2}+i \lambda\right)+R_{2}\left(\frac{n}{2}+i \lambda\right)\right)\right|_{\rho=0}= \\
\left.\left(R^{-\frac{n}{2}-i \lambda} \rho^{\prime \frac{n}{2}+i \lambda} F_{2}\left(\frac{n}{2}+i \lambda, \rho, \rho^{\prime}, R, \omega\right)\right)\right|_{\rho=0}
\end{gathered}
$$

Since $F_{2}\left(\frac{n}{2}+i \lambda, \bullet\right)$ is smooth, the restriction of $\rho^{\frac{n}{2}+i \lambda} F_{2}$ to $\rho=0$ is well defined.

Notice that the composition of the restriction to $\rho=0$ with the blow-down map $\beta$ gives a map

$$
\beta_{1}: \partial X \times_{0} X \longrightarrow \partial X \times X
$$

where $\partial X \times_{0} X$ denotes the manifold $\partial X \times X$ with the submanifold $\left\{x^{\prime}=0, y=y^{\prime}=0\right\}$ blown-up. By an abuse of notation we continue to use $R$ and $\rho^{\prime}$ to denote the restrictions of the corresponding functions to $\rho=0$. In other words we consider

$$
R=\left[x^{\prime 2}+\left|y-y^{\prime}\right|^{2}\right]^{\frac{1}{2}}, \quad \rho^{\prime}=\frac{x^{\prime}}{R} \omega=\frac{y-y^{\prime}}{R}
$$

Therefore (6.9) above gives a well defined distribution on the manifold $\partial X \times_{0} X$, namely

$$
F=\left.R^{-i \lambda} \rho^{\prime \frac{n}{2}+i \lambda} F_{2}\left(\frac{n}{2}+i \lambda, \rho, \rho^{\prime}, R, \omega\right)\right|_{\rho=0}
$$

By switching the variables $x$ and $x^{\prime}$ in the proof of Proposition 4.1 of [28] it can be shown that the push forward of $F$ to $\partial X \times X$ under the map $\beta_{1}$ is well defined. In view of (6.7) and (6.8) this is essentially the same thing we want to do here. Therefore the restriction $\left.x^{-\frac{n}{2}-i \lambda} R\left(\frac{n}{2}+i \lambda, z, z^{\prime}\right)\right|_{x=0}$ is well defined and we shall denote it by

$$
\left.E\left(\frac{n}{2}+i \lambda, y, z^{\prime}\right) \stackrel{\text { def }}{=} x^{-\frac{n}{2}-i \lambda} R\left(\frac{n}{2}+i \lambda, z, z^{\prime}\right)\right|_{x=0}=\beta_{1 *} F, \quad \lambda \in \mathbb{C} .
$$

It is clear from (6.8) that $E\left(\frac{n}{2}+i \lambda, y, z^{\prime}\right)$ has a conormal singularity at $\left\{x^{\prime}=0, y=y^{\prime}\right\}$. This is the transpose of the Eisenstein function, or Poisson operator, see for example [18, 20, 28]. 
This defines a meromorphic family of operators

$$
\begin{gathered}
E\left(\frac{n}{2}+i \lambda\right): C_{0}^{\infty}(\stackrel{\circ}{X}) \longrightarrow C^{\infty}(\partial X) \\
E\left(\frac{n}{2}+i \lambda\right) f(y)=\int_{X} E\left(\frac{n}{2}+i \lambda, y, z^{\prime}\right) f\left(z^{\prime}\right) d \operatorname{vol}_{g}\left(z^{\prime}\right), \quad \lambda \in \mathbb{C} \backslash \frac{i}{2} \mathbb{N} .
\end{gathered}
$$

So we conclude from (6.10) and (6.3) that

$$
\begin{gathered}
\int_{\mathbb{R}} e^{-i \lambda s} \mathcal{R}_{+}\left(f_{1}, f_{2}\right)(y, s) d s=\left.\int_{\mathbb{R}} x^{-\frac{n}{2}}\left(D_{s} V\right)(s-\log x, z) e^{-i \lambda s} d s\right|_{x=0}= \\
i \lambda \int_{X} E\left(\frac{n}{2}+i \lambda, y, z^{\prime}\right)\left(f_{2}\left(z^{\prime}\right)+\lambda f_{1}\left(z^{\prime}\right)\right) d \operatorname{vol}_{g}\left(z^{\prime}\right), \\
\Im \lambda<-\frac{n}{2} \text { if } \quad f_{1}, f_{2} \in C_{0}^{\infty}(X), \quad \text { and } \quad \Im \lambda \leq 0 \quad \text { if } \quad f_{1}, f_{2} \in C_{0}^{\infty}(X) \cap E_{\mathrm{ac}}(X) .
\end{gathered}
$$

In view of (6.11) the right hand side of this equation has a meromorphic continuation to $\mathbb{C} \backslash \frac{i}{2} \mathbb{N}$, so the left hand side can be meromorphically continued to $\mathbb{C} \backslash \frac{i}{2} \mathbb{N}$.

Recall from (6.4) that $\mathcal{R}_{+}\left(f_{1}, f_{2}\right) \in L^{2}(\mathbb{R} \times \partial X)$ if $\left(f_{1}, f_{2}\right) \in E_{\mathrm{ac}}(X)$. Thus the left hand side of (6.12) is well defined when $\Im \lambda=0$ for such initial data. We want to understand the extension of the right hand side for this type of data, and we proceed as in [18. An application of Green's identity, see for example the proof of Proposition 2.1 of [18], gives

$$
R\left(\frac{n}{2}+i \lambda, z, z^{\prime}\right)-R\left(\frac{n}{2}-i \lambda, z, z^{\prime}\right)=-2 i \lambda \int_{\partial X} E\left(\frac{n}{2}+i \lambda, y, z\right) E\left(\frac{n}{2}-i \lambda, y, z^{\prime}\right) d \operatorname{vol}_{h_{0}}(y),
$$

provided $\frac{n}{2} \pm i \lambda$ are not poles of the resolvent $R\left(\bullet, z, z^{\prime}\right)$.

On the other hand, by using (6.13) and Stone's formula, see the proof of Proposition 2.2 of [18, we deduce that the map

$$
\begin{gathered}
E: C_{0}^{\infty}(\stackrel{\circ}{X}) \longrightarrow C^{\infty}\left(\partial X \times \mathbb{R}_{+}\right) \\
\phi \longmapsto \sqrt{\frac{2}{\pi}} \int_{X} E\left(\frac{n}{2}+i \lambda, y, z^{\prime}\right) \phi\left(z^{\prime}\right) d \operatorname{vol}_{g}\left(z^{\prime}\right), \quad \lambda>0
\end{gathered}
$$

induces a surjective isometry of the space of absolute continuity of the Laplacian

$$
E: L_{\mathrm{ac}}^{2}(X) \longrightarrow L^{2}\left(\mathbb{R}^{+} ; L^{2}(\partial X), \lambda^{2} d \lambda\right) .
$$

Moreover it is an spectral representation in the sense that

$$
E \Delta=\left(\frac{n^{2}}{4}+\lambda^{2}\right) E
$$

A similar analysis can be carried out for the backward radiation field. We know that

$$
R\left(\frac{n}{2}-i \lambda\right)=\left(\Delta-\lambda^{2}-\frac{n^{2}}{4}\right)^{-1}=\int_{\mathbb{R}} U_{-}\left(t, z, z^{\prime}\right) e^{-i \lambda t} d t, \quad \Im \lambda>\frac{n}{2},
$$

and hence

$$
\begin{gathered}
\mathcal{F}\left(\mathcal{R}_{-}\left(f_{1}, f_{2}\right)\right)(y, \lambda)=i \lambda E\left(\frac{n}{2}-i \lambda\right)\left(f_{2}+\lambda f_{1}\right), \\
\Im \lambda>\frac{n}{2} \quad \text { if } \quad\left(f_{0}, f_{1}\right) \in C_{0}^{\infty}(X), \quad \Im \lambda \geq 0 \quad \text { if } \quad\left(f_{0}, f_{1}\right) \in C_{0}^{\infty}(X) \cap E_{\mathrm{ac}}(X) .
\end{gathered}
$$

Now we are ready to prove Theorem 5.1 
Proof. Using (6.14) we observe that, for $\lambda \in(0, \infty)$ and $f, g \in L_{\mathrm{ac}}^{2}(X)$,

$$
\langle f, g\rangle_{L^{2}(X)}=\frac{2}{\pi}\left\langle\lambda E\left(\frac{n}{2} \pm i \lambda\right) f, \lambda E\left(\frac{n}{2} \pm i \lambda\right) g\right\rangle_{L^{2}\left(\mathbb{R}_{+} \times \partial X\right)} .
$$

On the other hand, as $\overline{E\left(\frac{n}{2}+i \lambda\right)}=E\left(\frac{n}{2}-i \lambda\right)$ when $\lambda \in(0, \infty)$,

$$
\begin{gathered}
\left\|E\left(\frac{n}{2}+i \lambda\right)\left(\lambda f_{2}+\lambda^{2} f_{1}\right)\right\|_{L^{2}(\mathbb{R} \times \partial X)}^{2}= \\
\int_{-\infty}^{\infty} E\left(\frac{n}{2}+i \lambda\right)\left(\lambda f_{2}+\lambda^{2} f_{1}\right) E\left(\frac{n}{2}-i \lambda\right)\left(\lambda \bar{f}_{2}+\lambda^{2} \bar{f}_{1}\right) d \lambda d \operatorname{vol}_{h_{0}}= \\
\int_{0}^{\infty} E\left(\frac{n}{2}+i \lambda\right)\left(\lambda f_{2}+\lambda^{2} f_{1}\right) E\left(\frac{n}{2}-i \lambda\right)\left(\lambda \bar{f}_{2}+\lambda^{2} \bar{f}_{1}\right) d \lambda d \operatorname{vol}_{h_{0}}+ \\
\int_{0}^{\infty} E\left(\frac{n}{2}-i \lambda\right)\left(-\lambda f_{2}+\lambda^{2} f_{1}\right) E\left(\frac{n}{2}+i \lambda\right)\left(-\lambda \bar{f}_{2}+\lambda^{2} \bar{f}_{1}\right) d \lambda d \operatorname{vol}_{h_{0}}= \\
2 \int_{0}^{\infty}\left|E\left(\frac{n}{2}+i \lambda\right) \lambda f_{2}\right|^{2} d \lambda d \operatorname{vol}_{h_{0}}+2 \int_{0}^{\infty}\left|E\left(\frac{n}{2}+i \lambda\right) \lambda^{2} f_{1}\right|^{2} d \lambda d \operatorname{vol}_{h_{0}} .
\end{gathered}
$$

Equation (6.17) shows that

$$
\int_{0}^{\infty}\left|E\left(\frac{n}{2}+i \lambda\right) \lambda f_{2}\right|^{2} d \lambda d \operatorname{vol}_{h_{0}}=\frac{\pi}{2}\left\|f_{2}\right\|_{L^{2}(X)}^{2}
$$

But by 6.15

$$
\lambda^{2} E\left(\frac{n}{2}+i \lambda\right) f_{1}=E\left(\frac{n}{2}+i \lambda\right)\left(\Delta-\frac{n^{2}}{4}\right) f_{1}
$$

and thus

$$
\int_{0}^{\infty}\left|E\left(\frac{n}{2}+i \lambda\right) \lambda^{2} f_{1}\right|^{2} d \lambda d \operatorname{vol}_{h_{0}}=\left\langle\lambda E\left(\frac{n}{2}+i \lambda\right) f_{1}, \lambda E\left(\frac{n}{2}+i \lambda\right)\left(\Delta-\frac{n^{2}}{4}\right) f_{1}\right\rangle_{L^{2}(\mathbb{R} \times \partial X)} .
$$

Again by (6.17)

$$
\begin{gathered}
\left\langle\lambda E\left(\frac{n}{2}+i \lambda\right) f_{1}, \lambda E\left(\frac{n}{2}+i \lambda\right)\left(\Delta-\frac{n^{2}}{4}\right) f_{1}\right\rangle_{L^{2}(\mathbb{R} \times \partial X)}=\frac{\pi}{2}\left\langle f_{1},\left(\Delta-\frac{n^{2}}{4}\right) f_{1}\right\rangle_{L^{2}(X)}= \\
\frac{\pi}{2} \int_{X}\left(\left|d f_{1}\right|^{2}-\frac{n^{2}}{4}\left|f_{1}\right|^{2}\right) d \operatorname{vol}_{g} .
\end{gathered}
$$

So we conclude that for $\left(f_{1}, f_{2}\right) \in E_{\mathrm{ac}}(X)$,

$$
\left\|E\left(\frac{n}{2}+i \lambda\right)\left(\lambda f_{2}-\lambda^{2} f_{1}\right)\right\|_{L^{2}(\mathbb{R} \times \partial X)}^{2}=2 \pi\left\|\left(f_{1}, f_{2}\right)\right\|_{H_{E}(X)}^{2}
$$

Plancherel's theorem and (6.12) show that $\mathcal{R}_{+}$is norm preserving, and therefore its range is closed. It is clearly dense, otherwise there would be $\phi \in L^{2}(\mathbb{R} \times \partial X)$ orthogonal to the range of $\mathcal{R}$, but by (6.14), $\phi=0$. This concludes the proof of Theorem 5.1

In view of equations (6.12) and (6.16), Theorem 5.2 is equivalent to

Proposition 6.6. For $\lambda \in \mathbb{R}, \lambda \neq 0$, the scattering matrix is the unitary operator

$$
A(\lambda): L^{2}(\partial X) \longrightarrow L^{2}(\partial X)
$$

that satisfies

$$
E\left(\frac{n}{2}+i \lambda\right)=A(\lambda) E\left(\frac{n}{2}-i \lambda\right) .
$$


Proof. We need three things: equations (6.10), 6.13), and the expansion for $E\left(\frac{n}{2}+i \lambda, y, x^{\prime}, y^{\prime}\right)$ as $x^{\prime} \rightarrow 0$, which can be found in Propositions 4.1 and 4.2 of [28, i.e

$$
E\left(\frac{n}{2}+i \lambda, y, x^{\prime}, y^{\prime}\right)=\frac{1}{2 i \lambda} x^{\prime-i \lambda} \delta\left(y, y^{\prime}\right)+\frac{1}{2 i \lambda} x^{\prime i \lambda} A(\lambda)\left(y, y^{\prime}\right)+o(x), \quad \text { as } x^{\prime} \downarrow 0 .
$$

Now multiplying (6.13) by $x^{\prime-i \lambda}$ and applying (6.10) and (6.19), the result follows.

This is a known argument and can be found for example in the proofs of Proposition 2.5 of [20], or Corollary 2.6 of [18], which deal with Riemann surfaces. Here we used the results of [28] where needed.

\section{THE SUPPORT THEOREM}

The main goal of this section is to prove

Theorem 7.1. If $f \in L_{\mathrm{ac}}^{2}(X)$ and $\mathcal{R}_{+}(0, f)(s, y)$ is supported in $s \geq s_{0}$, with $s_{0}<<0$, then $f$ is supported in $\left\{x \geq e^{s_{0}}\right\}$.

Theorem [7.1] is a "support theorem" in the terminology of Helgason [23, 24] and is a generalization of Theorem 3.13 of [36, which is Theorem [7.1] for the hyperbolic space $\mathbb{H}^{3}$. Helgason [21, 24] proved such a result for functions that are compactly supported, but for more general symmetric spaces.

It is important to observe, as emphasized by Lax in [33, that this theorem does not have an analogue in (asymptotically) Euclidean space. In that case the function $f$ has to be rapidly decaying at infinitysee Theorem 2.6 and Remark 2.9 of [24] - which would correspond to infinite order of vanishing of $f$ at $x=0$. Here the only requirement is that $f \in L_{\mathrm{ac}}^{2}(X)$. However, in coordinates (2.4) the distance along a geodesic that approaches $\partial X$ perpendicularly is $-\log x$, so the requirement that $f \in L^{2}(X)$ already imposes an exponential of decay of $f$ near the boundary.

The first step in the proof of Theorem [7.1] is

Lemma 7.2. If $f \in L_{\mathrm{ac}}^{2}(X)$ and $\mathcal{R}_{+}(0, f)(s, y)$ is supported in $s \geq s_{0}$, then $f$ is compactly supported.

Proof. Without loss of generality we may assume that $f \in C^{\infty}(\stackrel{\circ}{X})$. Indeed, we recall from equations (6.12) and (6.15) that the Fourier transform in $s$ of the forward radiation field is a spectral representation of $\Delta-\frac{n^{2}}{4}$. Then if $\mathcal{F}$ denotes the Fourier transform in the $s$ variable then

$$
q\left(\lambda^{2}\right) \mathcal{F} \mathcal{R}_{+}(0, f)(\lambda, y)=\mathcal{F} \mathcal{R}_{+}\left(0, q\left(\Delta-\frac{n^{2}}{4}\right) f\right), \quad f \in L_{\mathrm{ac}}^{2}(X), \quad \forall q \in \mathcal{S}(\mathbb{R}) .
$$

If $\phi \in C_{0}^{\infty}(\mathbb{R})$, is an even function, which we choose to be supported in $(-\epsilon, \epsilon)$, then its Fourier transform $\widehat{\phi}$ is also an even function, and there exists a smooth function $\psi_{1} \in \mathcal{S}(\mathbb{R})$ such that

$$
\widehat{\phi}(\lambda)=\psi_{1}\left(\lambda^{2}\right) .
$$

Therefore by (7.1)

$$
\phi * \mathcal{R}_{+}(0, f)=\mathcal{R}_{+}\left(0, \psi_{1}\left(\Delta-\frac{n^{2}}{4}\right) f\right) .
$$

As $\frac{\partial^{2 k}}{\partial s^{2 k}} \phi * \mathcal{R}_{+}(0, f) \in L^{2}(\mathbb{R} \times \partial X), k=1,2, \ldots$, then

$$
\left(\Delta-\frac{n^{2}}{4}\right)^{k} \psi_{1}\left(\Delta-\frac{n^{2}}{4}\right) f \in L^{2}(X) .
$$


$\Delta-\frac{n^{2}}{4}$ is a standard elliptic operator in the interior of $X$, so $\psi_{1}\left(\Delta-\frac{n^{2}}{4}\right) f \in C^{\infty}(\stackrel{\circ}{X})$. Moreover, by elliptic regularity for totally characteristic operators, see Theorem 3.8 of [41,

$$
\left(x D_{x}, x D_{y_{1}}, \ldots, x D_{y_{n}}\right)^{\alpha} \psi_{1}\left(\Delta-\frac{n^{2}}{4}\right) f \in L^{2}(X), \quad \alpha \in \mathbb{N}^{n+1} .
$$

If $\phi \in C_{0}^{\infty}(\mathbb{R})$ is even, and $\mathcal{R}_{+}(0, f)(s, y)=0$ for $s<s_{0}, \phi * \mathcal{R}_{+}(0, f)(s, y)=0$ for $s<s_{0}-\epsilon$. So from now on we will assume that $f$, instead of $\psi_{1}\left(\Delta-\frac{n^{2}}{4}\right) f$, satisfies (7.4). Since the solution to the Cauchy problem is smooth for all (finite) times, the solution $V$ to (4.8) is smooth in $(0, T) \times(0, T) \times \partial X$. We do not know a priori that $V$ is smooth up to $\left\{x^{\prime}=0\right\} \cup\left\{t^{\prime}=0\right\}$. We proved in Theorem 2.1] that this is true if $f \in C_{0}^{\infty}(\stackrel{\circ}{X})$, but here $f$ is not yet known to be compactly supported and may be singular at $\left\{x^{\prime}=t^{\prime}=0\right\}$.

To illustrate our method, let us assume for a moment that $V$ is smooth up to $\left\{x^{\prime}=0\right\} \cup\left\{t^{\prime}=0\right\}$. Using the equation, and that $V\left(x^{\prime}, x^{\prime}, y\right)=0$, one can prove that if $\mathcal{R}_{+}(0, f)(s, y)=0$ for $s<s_{0}$, then all derivatives of $V$ vanish at $\left\{x^{\prime}=0\right\} \cup\left\{t^{\prime}=0\right\}$. Therefore we can extend $V$ smoothly across $\left\{t^{\prime}=0\right\} \cup\left\{x^{\prime}=0\right\}$ as $V=0$. We then want to use a uniqueness theorem to conclude that $V=0$ near $\left\{x^{\prime}=t^{\prime}=0\right\}$. In particular this will imply that $f(x, y)=0$ in a neighborhood of $\{x=0\}$ and we will be done.

The operator $P^{\prime}$ in (4.8) extends (although not uniquely) to a neighborhood of $\left\{x^{\prime}=t^{\prime}=0\right\}$, however notice that the coefficients of the terms of (4.8) involving second order derivatives in $y$ vanish to second order at $x^{\prime}=t^{\prime}=0$, so Hörmander's uniqueness theorem cannot be used to guarantee that $V=0$ near $\left\{t^{\prime}=x^{\prime}=0\right\}$. The uniqueness theorem which deals with the Cauchy problem for second order operators with this type of degeneracy is due to Alinhac, Theorem 1.1.2 of [1. Notice that, although $P^{\prime}$ is real, it is not of principal type at $\left\{x^{\prime}=t^{\prime}=0\right\}$, so the result of Lerner and Robbiano, see 38] or Theorem 28.4.3 of [26], cannot be applied either.

The principal symbol of $P^{\prime}$ is

$$
p=\sigma_{2}\left(P^{\prime}\right)=-\xi \tau-x^{\prime} t^{\prime} h\left(x^{\prime} t^{\prime}, y, \eta\right), \quad h\left(x^{\prime} t^{\prime}, y, \eta\right)=\sum_{i j} h^{i j}\left(x^{\prime} t^{\prime}, y\right) \eta_{i} \eta_{j} .
$$

If $H_{p}$ denotes the Hamilton vector field of $p$ and $\phi=x^{\prime}+t^{\prime}$, then

$$
H_{p}=-\tau \frac{\partial}{\partial x^{\prime}}-\xi \frac{\partial}{\partial t^{\prime}}-x^{\prime} t^{\prime} H_{h}+\left(t^{\prime} h+x^{\prime} t^{\prime 2} h_{1}\right) \frac{\partial}{\partial \xi}+\left(x^{\prime} h+t^{\prime} x^{\prime 2} h_{1}\right) \frac{\partial}{\partial \tau},
$$

where $H_{h}$ denotes the Hamilton vector field of $h$ in the variables $y$ and $\eta$ only, and $h_{1}\left(x^{\prime} t^{\prime}, y, \eta\right)$ denotes the derivative of $h$ in the first variable. Hence

$$
H_{p}^{2} \phi=-\left(x^{\prime}+t^{\prime}\right)\left[h\left(x^{\prime} t^{\prime}, y, \eta\right)+x^{\prime} t^{\prime} h_{1}\left(x^{\prime} t^{\prime}, y, \eta\right)\right],
$$

Since $h(0, y, \eta)$ is non-degenerate, it follows that for $x^{\prime}, t^{\prime}$ small

$$
-\phi H_{p}^{2} \phi \geq \frac{1}{2} \phi^{2} h
$$

We can therefore apply Theorem 1.1.2 of [1] to $P^{\prime}$ and $\phi$ (with $\Lambda_{S}=\emptyset$, and $a=C=0$ as in the statement of Theorem 1.1.2 of [1]) to guarantee that $V=0$ in a neighborhood of $\left\{x^{\prime}=t^{\prime}=0\right\}$. See figure 3 In particular this shows that $f(x, y)=0$ near $x=0$, so $f \in C_{0}^{\infty}(\stackrel{\circ}{X})$, as we want to prove.

We emphasize that the key reason this works is the fact that the extension of $V$ would be supported in the wedge $\left\{x^{\prime} \geq 0, t^{\prime} \geq 0\right\}$, so the intersection of the support of $V$ and $\{\phi=0\}$ is compact, as required by Theorem 1.1.2 of [1].

The main difficulty to apply this method to prove Theorem [7.1] is that $V$ is not known to be smooth up to $\left\{x^{\prime}=0\right\} \cup\left\{t^{\prime}=0\right\}$, and Theorem 1.1.2 of [1] is proved for smooth functions. We have to show that 

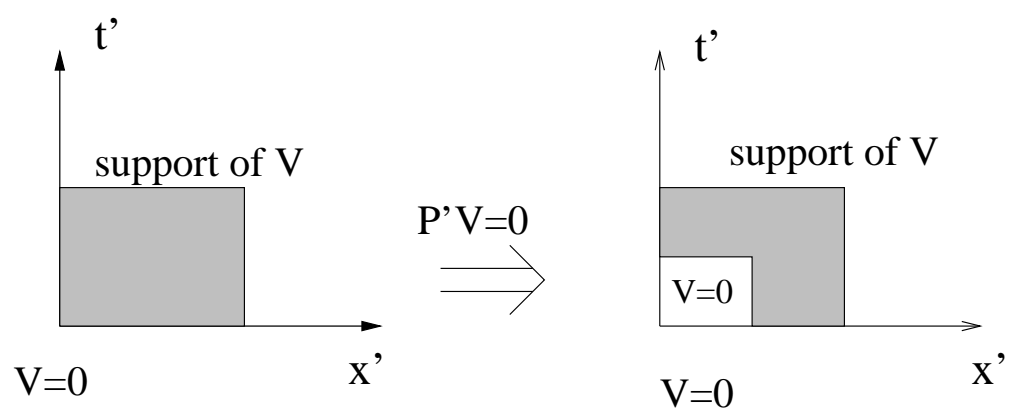

Figure 3. Unique continuation for $V . P^{\prime}$ is degenerate at $x^{\prime}=t^{\prime}=0$, but $V$ is supported in the wedge $\left\{x^{\prime} \geq 0, t^{\prime} \geq 0\right\}$

our assumptions guarantee enough regularity of $V$ to make Alinhac's argument work. Fortunately the operator $P^{\prime}$ in (4.8) is much simpler than the general case of [1], and the necessary Carleman estimates are relatively simple to obtain.

We will work with $W$, which is defined in (4.22), instead of $V$. The advantage is that $W$ satisfies (4.23) which has no first order derivatives in $x^{\prime}$ or $t^{\prime}$.

The proof is divided in two steps:

Step 1: We will use that $W\left(x^{\prime}, x^{\prime}, y\right)=0, x^{\prime}>0$, and $\mathcal{R}_{+}(0, f)=0$, to show that $W$ can be extended as a (distribution) solution to (4.23) in a neighborhood of $\left\{x^{\prime}=t^{\prime}=0\right\}$ vanishing when either $x^{\prime}<0$ or $t^{\prime}<0$.

Step 2: We use the method of proof of Theorem 1.1.2 of [1] to show that $W=0$ in a neighborhood of $\left\{x^{\prime}=t^{\prime}=0\right\}$.

Proof of Step 1. We will show that the conditions $W\left(x^{\prime}, x^{\prime}, y\right)=0, x^{\prime}>0$, and $\mathcal{R}(0, f)=0$ for $s<s_{0}$, imply that $W$ has an extension $\widetilde{W}$ satisfying

$$
\widetilde{W} \in H^{2 k}\left((-T, T) \times(-T, T) ; H^{-2 k}(\partial X)\right), \quad k \in \mathbb{N}
$$

and

$$
\begin{gathered}
\left(\frac{\partial^{2}}{\partial x^{\prime} \partial t^{\prime}}+x^{\prime} t^{\prime} \Delta_{h}+x^{\prime} t^{\prime} B\left(x^{\prime} t^{\prime}, y, \frac{\partial}{\partial y}\right)+C\left(x^{\prime} t^{\prime}, y\right)\right) \widetilde{W}=0 \text { in } \widetilde{\Omega} \\
\widetilde{W}\left(x^{\prime}, t^{\prime}, y\right)=W\left(x^{\prime}, t^{\prime}, y\right) \text { if } x^{\prime}>0, t^{\prime}>0 \\
\widetilde{W}\left(x^{\prime}, t^{\prime}, y\right)=0 \text { if } x^{\prime}<0, t^{\prime}<0 .
\end{gathered}
$$

Here we have extended the operators $\Delta_{h}, B$ and the coefficient $C$ smoothly across $\left\{x^{\prime}=0\right\} \cup\left\{t^{\prime}=0\right\}$.

It was proved in Lemma 4.1 that when $f \in L^{2}(X)$, and $T$ is small, the solution $W$ of (4.23) satisfies

$$
\begin{gathered}
\left.W, x^{\prime \frac{1}{2}} D_{x^{\prime}} W, t^{\frac{1}{2}} D_{t^{\prime}} W \in L^{2}([0, T] \times[0, T] \times \partial X)\right), \\
{\left[x^{\prime} t^{\prime}\left(x^{\prime}+t^{\prime}\right)\right]^{\frac{1}{2}} D_{y_{j}} W \in L^{2}([0, T] \times[0, T] \times \partial X), \quad 1 \leq j \leq n .}
\end{gathered}
$$

We emphasize that, due to the smoothness of $f, W$ is smooth in the region $\left\{x^{\prime}>0, t^{\prime}>0\right\}$.

We will show (7.7) for $k=1$. For that, let $\phi \in C^{\infty}(\partial X)$ and

$$
G\left(x^{\prime}, t^{\prime}\right)=\int_{\partial X} W\left(x^{\prime}, t^{\prime}, y\right) \phi(y) d y
$$

Using equation (4.23) and (7.9) we deduce that

$$
\frac{\partial^{2} W\left(x^{\prime}, t^{\prime}, y\right)}{\partial x^{\prime} \partial t^{\prime}} \in L^{2}\left([0, T] \times[0, T] ; H^{-1}(\partial X)\right) .
$$


Differentiating (4.23) in $x^{\prime}$ we have

$$
\begin{gathered}
\frac{\partial^{2}}{\partial x^{\prime 2}} \frac{\partial}{\partial t^{\prime}} W= \\
-t^{\prime} \Delta_{h} W-x^{\prime} t^{\prime} Q W-t^{\prime} \Delta_{h} x^{\prime} \frac{\partial}{\partial x^{\prime}} W-t^{\prime} B W-x^{\prime} t^{\prime} B_{1} W-t^{\prime} B x^{\prime} \frac{\partial}{\partial x^{\prime}} W-C_{1} W-C \frac{\partial}{\partial x^{\prime}} W,
\end{gathered}
$$

where $Q$ and $B_{1}$ are second and first order operators respectively, involving $y$ derivatives only. Using (7.9) we find that

$$
x^{\prime \frac{1}{2}} \frac{\partial^{2}}{\partial{x^{\prime}}^{2}} \frac{\partial}{\partial t^{\prime}} W \in L^{2}\left([0, T] \times[0, T] ; H^{-2}(\partial X)\right) .
$$

Since $W\left(t^{\prime}, t^{\prime}, y\right)=0, t^{\prime}>0$, equation (4.23) shows that $\frac{\partial}{\partial x^{\prime}} \frac{\partial}{\partial t^{\prime}} W\left(t^{\prime}, t^{\prime}, y\right)=0, t^{\prime}>0$ and thus we can write

$$
\frac{\partial^{2}}{\partial x^{\prime} \partial t^{\prime}} G\left(x^{\prime}, t^{\prime}\right)=-\int_{x^{\prime}}^{t^{\prime}} \frac{\partial^{2}}{\partial s^{2}} \frac{\partial}{\partial t^{\prime}} G\left(s, t^{\prime}\right) d s, \quad \text { if } t^{\prime}>x^{\prime}>0
$$

Therefore

$$
\left|\frac{\partial}{\partial x^{\prime}} \frac{\partial}{\partial t^{\prime}} G\left(x^{\prime}, t^{\prime}\right)\right|^{2}=\left|\int_{x^{\prime}}^{t^{\prime}} \frac{\partial^{2}}{\partial s^{2}} \frac{\partial}{\partial t^{\prime}} G\left(s, t^{\prime}\right) d s\right|^{2} \leq \log \left(\frac{t^{\prime}}{x^{\prime}}\right) \int_{x^{\prime}}^{t^{\prime}} s\left|\frac{\partial^{2}}{\partial s^{2}} \frac{\partial}{\partial t^{\prime}} G\left(s, t^{\prime}\right)\right|^{2} d s .
$$

So from (7.13) we obtain for $T$ small and $\delta<\frac{1}{2}$,

$$
\begin{gathered}
\int_{0}^{T} \int_{0}^{t^{\prime}} x^{\prime-2 \delta}\left|\frac{\partial}{\partial x^{\prime}} \frac{\partial}{\partial t^{\prime}} G\left(x^{\prime}, t^{\prime}\right)\right|^{2} d x^{\prime} d t^{\prime} \leq \\
\int_{0}^{T} \int_{0}^{t^{\prime}} \frac{s^{1-2 \delta}}{(1-2 \delta)}\left((2-2 \delta)|\log s|+\frac{1}{1-2 \delta}\right) s\left|\frac{\partial^{2}}{\partial s^{2}} \frac{\partial}{\partial t^{\prime}} G\left(s, t^{\prime}\right)\right|^{2} d s d t^{\prime}< \\
\left\|x^{\prime \frac{1}{2}} \frac{\partial^{2}}{\partial x^{\prime 2}} \frac{\partial}{\partial t^{\prime}} W\right\|_{L^{2}\left([0, T] \times[0, T] ; H^{-2}(\partial X)\right)}^{2}<\infty
\end{gathered}
$$

So

$$
x^{\prime-\delta} \frac{\partial}{\partial x^{\prime}} \frac{\partial}{\partial t^{\prime}} G \in L^{2}\left(\left\{t^{\prime} \geq x^{\prime}>0\right\}\right), \quad \delta<\frac{1}{2},
$$

with uniform bound up to $x^{\prime}=0$. This together with (7.13) imply that

$$
\frac{\partial}{\partial x^{\prime}}\left(x^{\prime \gamma} \frac{\partial}{\partial x^{\prime}} \frac{\partial}{\partial t^{\prime}} W\right) \in L^{2}\left(\left\{t^{\prime} \geq x^{\prime}>0\right\} ; H^{-2}(\partial X)\right), \quad \gamma>\frac{1}{2} .
$$

Thus the restriction $\left(x^{\prime \gamma} \frac{\partial}{\partial x^{\prime}} \frac{\partial}{\partial t^{\prime}} W\right)\left(0, t^{\prime}, y\right), t^{\prime}>0$, is well defined for any $\gamma>\frac{1}{2}$. In particular this shows that

$$
\left(x^{\prime} \frac{\partial}{\partial x^{\prime}} W\right)\left(0, t^{\prime}, y\right)=0, \quad \text { in }(0, T) \times \partial X .
$$

In view of (17.11), the restriction $\frac{\partial}{\partial t^{\prime}} W\left(0, t^{\prime}, y\right)$ is well defined.

Recall that in these coordinates, and in terms of $W$, the radiation field is given by

$$
\mathcal{R}_{+}(0, f)=\left.\frac{1}{2}|h|^{\frac{1}{4}}(0, y)\left(t^{\prime} \frac{\partial W}{\partial t^{\prime}}-x^{\prime} \frac{\partial W}{\partial x^{\prime}}\right)\right|_{x^{\prime}=0}=0
$$

So (7.17) and (7.16) show that

$$
\frac{\partial}{\partial t^{\prime}} W\left(0, t^{\prime}, y\right)=0, \quad \text { in } \quad(0, T) \times \partial X
$$

Again applying the regularity of $W$ given by (7.11) and using (7.18), we have for $t^{\prime}>0$, 


$$
\left|\frac{\partial G}{\partial t^{\prime}}\left(t^{\prime}, t^{\prime}\right)\right|^{2} \leq\left|\int_{0}^{t^{\prime}} \frac{\partial^{2} G}{\partial s \partial t^{\prime}}\left(s, t^{\prime}\right) d s\right|^{2} \leq \frac{t^{\prime \delta+1}}{\delta+1} \int_{0}^{t^{\prime}} s^{-\delta}\left|\frac{\partial^{2} G}{\partial s \partial t^{\prime}}\left(s, t^{\prime}\right)\right|^{2} d s, \quad \delta<\frac{1}{2},
$$

and we find that

$$
\int_{0}^{T} t^{\prime-1-\delta}\left|\frac{\partial G}{\partial t^{\prime}}\left(t^{\prime}, t^{\prime}\right)\right|^{2}<\infty, \quad \delta<\frac{3}{2}
$$

In particular this shows that

$$
\frac{\partial W}{\partial x^{\prime}}\left(x^{\prime}, x^{\prime}, y\right), \quad \frac{\partial W}{\partial t^{\prime}}\left(x^{\prime}, x^{\prime}, y\right) \in L^{2}\left([0, T] ; H^{-2}(\partial X)\right) .
$$

We can then write

$$
\begin{gathered}
\frac{\partial W}{\partial t^{\prime}}\left(x^{\prime}, t^{\prime}, y\right)=\frac{\partial W}{\partial t^{\prime}}\left(x^{\prime}, x^{\prime}, y\right)+\int_{x^{\prime}}^{t^{\prime}} \frac{\partial^{2} W}{\partial s \partial t^{\prime}}\left(s, t^{\prime}, y\right) d s \text { and } \\
\frac{\partial W}{\partial x^{\prime}}\left(x^{\prime}, t^{\prime}, y\right)=\frac{\partial W}{\partial x^{\prime}}\left(x^{\prime}, x^{\prime}, y\right)+\int_{x^{\prime}}^{t^{\prime}} \frac{\partial^{2} W}{\partial x^{\prime} \partial s}\left(x^{\prime}, s, y\right) d s
\end{gathered}
$$

and use (7.20) to show that

$$
\frac{\partial W}{\partial t^{\prime}}\left(x^{\prime}, t^{\prime}, y\right) \text { and } \frac{\partial W}{\partial x^{\prime}}\left(x^{\prime}, t^{\prime}, y\right) \in L^{2}\left([0, T] \times[0, T] H^{-2}(\partial X)\right) .
$$

Therefore $W\left(0, t^{\prime}, y\right), t^{\prime} \in[0, T]$, is well defined and, by (7.18), $W\left(0, t^{\prime}, y\right)=F(y)$. Since $W\left(x^{\prime}, x^{\prime}, y\right)=0$, and we want to construct a smooth extension to $G$, we must have

$$
W\left(0, t^{\prime}, y\right)=0, \quad t^{\prime}>0
$$

Substituting (7.21) back in (7.12) we deduce that

$$
\frac{\partial}{\partial x^{\prime}} \frac{\partial}{\partial x^{\prime}} \frac{\partial}{\partial t^{\prime}} W \in L^{2}\left([0, T] \times[0, T] ; H^{-2}(\partial X)\right)
$$

Therefore $\frac{\partial}{\partial x^{\prime}} \frac{\partial}{\partial t^{\prime}} W\left(0, t^{\prime}, y\right)$ is well defined and from (4.23) and (7.22)

$$
\frac{\partial}{\partial x^{\prime}} \frac{\partial}{\partial t^{\prime}} W\left(0, t^{\prime}, y\right)=0, \quad t^{\prime}>0 .
$$

But we need to prove that $\left.\frac{\partial W}{\partial x^{\prime}}\right|_{x^{\prime}=0}=0$ on $[0, T]$. To do this we take the derivative of (4.23) with respect to $t^{\prime}$.

$$
\begin{gathered}
\frac{\partial}{\partial x^{\prime}} \frac{\partial^{2}}{\partial t^{\prime 2}} W= \\
-x^{\prime} \Delta_{h} W-x^{\prime} t^{\prime} Q_{1} W-x^{\prime} \Delta_{h} t^{\prime} \frac{\partial}{\partial t^{\prime}} W-x^{\prime} B W-x^{\prime} t^{\prime} B_{2} W-x^{\prime} B t^{\prime} \frac{\partial}{\partial t^{\prime}} W-C_{2} W-C \frac{\partial}{\partial t^{\prime}} W,
\end{gathered}
$$

where as in (7.12), $Q_{1}$ and $B_{2}$ are second and first order operators respectively, involving $y$ derivatives only. Using (7.9) and (7.21) we find that

$$
\frac{\partial}{\partial x^{\prime}} \frac{\partial^{2}}{\partial t^{\prime 2}} W \in L^{2}\left([0, T] \times[0, T] H^{-2}(\partial X)\right) .
$$

Therefore (7.21), (7.23) and (7.26) imply that

$$
W \quad \text { and } \quad \frac{\partial}{\partial x^{\prime}} \frac{\partial}{\partial t^{\prime}} W \in H^{1}\left([0, T] \times[0, T] ; H^{-2}(\partial X)\right) .
$$


From (7.25) and (7.26) we deduce that $\left.\frac{\partial^{2} W}{\partial t^{\prime 2}}\left(0, t^{\prime}\right)\right|_{x^{\prime}=0}$ is well defined and by (7.18), $\left.\frac{\partial^{2} W}{\partial t^{\prime 2}}\left(0, t^{\prime}\right)\right|_{x^{\prime}=0}=0$. By symmetry $\frac{\partial^{2} W}{\partial x^{\prime 2}}\left(x^{\prime}, 0\right)=0$. Thus we can write

$$
\begin{aligned}
& \frac{\partial^{2} W}{\partial t^{\prime 2}}\left(x^{\prime}, t^{\prime}, y\right)=\int_{0}^{x^{\prime}} \frac{\partial}{\partial s} \frac{\partial^{2}}{\partial t^{\prime 2}} W d s \\
& \frac{\partial^{2} W}{\partial{x^{\prime}}^{2}}\left(x^{\prime}, t^{\prime}, y\right)=\int_{0}^{t^{\prime}} \frac{\partial}{\partial s} \frac{\partial^{2}}{\partial{x^{\prime}}^{2}} W d s .
\end{aligned}
$$

and then conclude that

$$
\frac{\partial^{2} W}{\partial{t^{\prime}}^{2}}\left(x^{\prime}, t^{\prime}, y\right), \quad \frac{\partial^{2} W}{\partial{x^{\prime}}^{2}}\left(x^{\prime}, t^{\prime}, y\right) \in L^{2}\left([0, T] \times[0, T] ; H^{-2}(\partial X)\right) .
$$

From (7.9) and (7.21) we have

$$
W \in H^{2}\left([0, T] \times[0, T] ; H^{-2}(\partial X)\right) .
$$

Moreover $W=0$ on $\left\{x^{\prime}=0\right\} \cup\left\{t^{\prime}=0\right\}$.

Now we write

$$
\frac{\partial^{2} G}{\partial t^{\prime 2}}\left(t^{\prime}, t^{\prime}\right)=\int_{0}^{t^{\prime}} \frac{\partial}{\partial s} \frac{\partial^{2} G}{\partial t^{\prime 2}}\left(s, t^{\prime}\right) d s
$$

As in the proof of (7.19) we use (17.26) to show that $G\left(t^{\prime}, t^{\prime}\right) \in C^{1}([0, T])$ and, by symmetry,

$$
\frac{\partial G}{\partial t^{\prime}}(0,0)=\frac{\partial G}{\partial x^{\prime}}(0,0)=0 .
$$

From (7.27) $\frac{\partial W}{\partial t^{\prime}}\left(0, t^{\prime}, y\right) \in H^{1}\left([0, \epsilon] ; H^{-2}(\partial X)\right)$, From this and [7.18) we deduce that

$$
\frac{\partial W}{\partial t^{\prime}}\left(0, t^{\prime}, y\right)=0, \quad \frac{\partial W}{\partial x^{\prime}}\left(x^{\prime}, 0, y\right)=0, \quad \text { in } \quad[0, T] \times \partial X .
$$

This and the regularity of $W$ given by (7.28) are enough to guarantee that if $\widetilde{W}\left(x^{\prime}, t^{\prime}, y\right)$ is the extension of $W$ to $(-T, T) \times(-T, T) \times \partial X$, with $\widetilde{W}=0$ in $\left\{x^{\prime}<0\right\} \cup\left\{t^{\prime}<0\right\}$, then it satisfies (7.8) and

$$
\widetilde{W} \in H^{2}\left([-T, T] \times[-T, T] ; H^{-2}(\partial X)\right) .
$$

To prove (7.7) we substitute the regularity (7.30) back into (4.23) and iterate following the argument above. This also shows that all derivatives of $\widetilde{W}$ vanish at $\left\{x^{\prime}=0\right\} \cup\left\{t^{\prime}=0\right\}$. This ends the proof of step 1.

Proof of step 2: We want to show that (7.7) and (7.9) are enough to apply Alinhac's theorem.

First we make the change of variables

$$
t^{\prime}=\mu+\nu, \quad x^{\prime}=\mu-\nu
$$

From (4.23) $\widetilde{U}(u, v, y)=\widetilde{W}(\mu+\nu, \mu-\nu, y)$ satisfies

$$
\begin{gathered}
P \widetilde{U}=0, \quad \text { where } \\
P=\frac{\partial^{2}}{\partial \mu^{2}}-\frac{\partial^{2}}{\partial \nu^{2}}+4\left(\mu^{2}-\nu^{2}\right) \sum_{i, j=1}^{n} h^{i j}\left(\mu^{2}-\nu^{2}, y\right) \frac{\partial}{\partial y_{i}} \frac{\partial}{\partial y_{j}}+\left(\mu^{2}-\nu^{2}\right) \sum_{j=1}^{n} b_{j}\left(\mu^{2}-\nu^{2}, y\right) \frac{\partial}{\partial y_{j}}+ \\
C\left(\mu^{2}-\nu^{2}, y\right)
\end{gathered}
$$


As in section 4.1 of [1], let

$$
\begin{gathered}
Q=-\frac{\partial^{2}}{\partial \nu^{2}}+4\left(\mu^{2}-\nu^{2}\right) \sum_{i, j=1}^{n} h^{i j}\left(\mu^{2}-\nu^{2}, y\right) \frac{\partial}{\partial y_{i}} \frac{\partial}{\partial y_{j}}, \\
L=\frac{\partial^{2}}{\partial \mu^{2}}+Q \quad \text { and } \quad A=L+\frac{\gamma(\gamma-1)}{\mu^{2}} \text { with } \gamma \geq \frac{1}{10} .
\end{gathered}
$$

We could have taken $\gamma>\gamma_{0}>0$, but to avoid one more parameter we set $\gamma_{0}=\frac{1}{10}$, which is good enough for our purposes. Let $Y_{0} \subset \partial X$ be a neighborhood $y_{0} \in \partial X$ and let $C_{0}^{* \infty}\left([0, T)_{\mu} \times(-T, T)_{\nu} \times Y_{0}\right)$ denote the set of functions in $C_{0}^{\infty}\left([0, T)_{\mu} \times(-T, T)_{\nu} \times Y_{0}\right)$ which vanish to infinite order at $\{\mu=0\}$. Integration by parts gives that for every $v \in C_{0}^{* \infty}\left([0, T)_{\mu} \times(-T, T)_{\nu} \times Y_{0}\right)$,

$$
\begin{gathered}
\left\|\mu^{-\gamma} L \mu^{\gamma} v\right\|^{2}=-2 \gamma \Re\left(\mu Q_{\mu} v, \frac{v}{\mu^{2}}\right)+4\left(\gamma^{2}+\gamma\right)\left\|\mu^{-1} v_{\mu}\right\|^{2}+\|A v\|^{2}+\left(4 \gamma^{3}-4 \gamma^{2}-6 \gamma\right)\left\|\mu^{-2} v\right\|^{2} \\
-2 \gamma\left(A v, \mu^{-2} v\right) .
\end{gathered}
$$

Here $Q_{\mu}$ is the operator obtained by differentiating the coefficients of $Q$ with respect to $\mu$, and $\|$, denotes the norm in $L^{2}\left((-T, T) \times(-T, T) \times Y_{0}\right)$. This is equation 4.11 of [1] with $a=0$ and $a^{\prime}=1$.

Hardy's inequality, see for example Lemma 5.3.1 of [7], gives that $\left\|\mu^{-1} v_{\mu}\right\|^{2} \geq \frac{9}{4}\left\|\mu^{-2} v\right\|^{2}$. Using this and applying Cauchy-Schwartz inequality to the last term of (7.31) gives

$$
\left\|\mu^{-\gamma} L \mu^{\gamma} v\right\|^{2} \geq-2 \gamma \Re\left(\mu Q_{\mu} v, \mu^{-2} v\right)+2 \gamma^{2}\left\|\mu^{-1} v_{\mu}\right\|^{2}+\left(4 \gamma^{3}+\frac{1}{2} \gamma^{2}+2 \gamma\right)\left\|\mu^{-2} v\right\|^{2} .
$$

The derivate of $Q$ with respect to $\mu$ is

$$
Q_{\mu}=8 \mu \sum_{i, j=1}^{n} h^{i j}\left(\mu^{2}-\nu^{2}, y\right) \frac{\partial}{\partial y_{i}} \frac{\partial}{\partial y_{j}}+8 \mu\left(\mu^{2}-\nu^{2}\right) \sum_{i, j=1}^{n} h_{1}^{i j}\left(\mu^{2}-\nu^{2}, y\right) \frac{\partial}{\partial y_{i}} \frac{\partial}{\partial y_{j}},
$$

where $h_{1}$ denotes the derivative of $h$ in the first variable. Since $h(0, y)$ is non-degenerate, then for $T$ small enough,

$$
\Re\left(\mu Q_{\mu} v, \mu^{-2} v\right) \leq-C\left\|\nabla_{y} v\right\|^{2} .
$$

Letting $\mu^{\gamma} v=w$, using (7.32) and (7.33) we arrive at the following Carleman estimate for the operator $L$.

$$
\begin{aligned}
\left\|\mu^{-\gamma} L w\right\|^{2} \geq & C \gamma\left\|\mu^{-\gamma} \nabla_{y} w\right\|^{2}+\gamma^{2}\left\|\mu^{-1}\left(\mu^{-\gamma} w\right)_{\mu}\right\|^{2}+\gamma^{3}\left\|\mu^{-2-\gamma} w\right\|^{2}, \\
& \text { for all } w \in C_{0}^{* \infty}\left([0, T)_{\mu} \times(-T, T)_{\nu} \times Y_{0}\right) .
\end{aligned}
$$

By the definition of $P$ we have that, for $T$ small, there exists $K$ depending on $C, T$, but not on $\gamma$ such that

$$
\begin{aligned}
K\left\|\mu^{-\gamma} P w\right\|^{2} & \geq \gamma\left\|\mu^{-\gamma} \nabla_{y} w\right\|^{2}+\gamma^{2}\left\|\mu^{-1}\left(\mu^{-\gamma} w\right)_{\mu}\right\|^{2}+\gamma^{3}\left\|\mu^{-2-\gamma} w\right\|^{2}, \\
& \text { for all } w \in C_{0}^{* \infty}\left([0, T)_{\mu} \times(-T, T)_{\nu} \times Y_{0}\right) .
\end{aligned}
$$

Let

$$
\chi(y) \in C_{0}^{\infty}\left(\mathbb{R}^{n}\right), \quad \chi(0)=1, \quad \int_{\mathbb{R}^{n}} \chi(y) d y=1 \text { and } \chi_{\delta}(y)=\delta^{-n} \chi\left(\frac{y}{\delta}\right), \quad \delta>0,
$$

and for $y_{0} \in Z_{0} \subset \subset Y_{0}$, let

$$
\phi \in C_{0}^{\infty}\left((-T, T)_{\mu} \times(-T, T)_{\nu} \times Y_{0}\right), \text { with } \phi(\mu, \nu, y)=1 \text { in }\left(-\frac{T}{2}, \frac{T}{2}\right)_{\mu} \times\left(-\frac{T}{2}, \frac{T}{2}\right)_{\nu} \times Z_{0} .
$$


Let $v_{\delta}=\chi_{\delta} *^{\prime} \phi \widetilde{U}$, where as in [25], $*^{\prime}$ means that the convolution is taken in the variable $y$ only. In view of (7.7) and the fact that all derivatives of $\widetilde{W}$ vanish at $\left\{x^{\prime}=0\right\} \cup\left\{t^{\prime}=0\right\}, v_{\delta} \in C_{0}^{* \infty}\left([0, T)_{\mu} \times(-T, T)_{\nu} \times Y_{0}\right)$, for $\delta$ small enough. Therefore we can apply (7.34) to $v_{\delta}$. Thus

$$
K\left\|\mu^{-\gamma} P v_{\delta}\right\|^{2} \geq \gamma\left\|\mu^{-\gamma} \nabla_{y} v_{\delta}\right\|^{2}+\gamma^{2}\left\|\mu^{-1}\left(\mu^{-\gamma} v_{\delta}\right)_{\mu}\right\|^{2}+\gamma^{3}\left\|\mu^{-2-\gamma} v_{\delta}\right\|^{2} .
$$

Now we want to take the limit of (7.35) as $\delta \rightarrow 0$. First we observe that, since $\widetilde{U}$ is supported in $\mu \geq|\nu|$ and satisfies $P \widetilde{U}=0$, and $\phi=1$ near $\mu=\nu=0$, it follows that $\mu>\frac{1}{2} T$ on the support of $P \phi \widetilde{U}$. From (7.9) we have

$$
\left\|\mu^{-\gamma} P \phi \widetilde{U}\right\|^{2} \leq\left(\frac{1}{2} T\right)^{-\gamma}\|P \phi \widetilde{U}\|^{2}<\infty, \quad \forall \gamma>\frac{1}{10} .
$$

On the other hand we write

$$
P v_{\delta}=\chi_{\delta} *^{\prime}(P \phi \widetilde{U})+\left[P, \chi_{\delta} *^{\prime}\right] \phi \widetilde{U}
$$

and observe that

$$
\begin{gathered}
{\left[P, \chi_{\delta} *^{\prime}\right]=} \\
{\left[\sum_{i, j}\left(\left(\mu^{2}-\nu^{2}\right)\left(h^{i j}\left(\mu^{2}-\nu^{2}, y\right) \frac{\partial}{\partial y_{i}} \frac{\partial}{\partial y_{j}}+b_{j}\left(\mu^{2}-\nu^{2}, y\right) \frac{\partial}{\partial y_{j}}\right)+\left(\mu^{2}-\nu^{2}\right) B_{1}\left(\mu^{2}-\nu^{2}, y\right)\right), \chi_{\delta} *^{\prime}\right] .}
\end{gathered}
$$

Equation 2.4.18 and Theorem 2.4.3 of of [25] show that, for $u \in H^{s-1}\left(\mathbb{R}^{n}\right)$ and $a \in C_{0}^{\infty}\left(\mathbb{R}^{n}\right)$,

$$
\begin{aligned}
& \left\|a\left(u * \chi_{\delta}\right)-(a u) * \chi_{\delta}\right\|_{H^{s}} \leq C\|u\|_{H^{s-1}}, \quad \text { and } \\
& a\left(u * \chi_{\delta}\right)-(a u) * \chi_{\delta} \rightarrow 0, \quad \text { in } H^{s}\left(\mathbb{R}^{n}\right) \text { as } \delta \rightarrow 0 .
\end{aligned}
$$

Applying this to our case, it follows from (7.37) that, if

$$
\left\|\mu^{-\gamma}\left(\mu^{2}-\nu^{2}\right) \nabla_{y}(\phi \widetilde{U})\right\|<\infty, \text { and }\left\|\mu^{-\gamma}\left(\mu^{2}-\nu^{2}\right) \phi \widetilde{U}\right\|_{L^{2}\left([0, T] \times[0, T] ; H^{-1}\left(Y_{0}\right)\right)}<\infty
$$

then

$$
\lim _{\delta \rightarrow 0}\left\|\mu^{-\gamma}\left[P, \chi_{\delta}^{\prime}\right](\phi \widetilde{U})\right\|=0 .
$$

Thus, if $\phi \widetilde{U}$ satisfies (17.38) for a certain $\gamma>\frac{1}{10}$, it follows from (7.36) that we can take the limit as $\delta \rightarrow 0$ in (7.35) and therefore

$$
\infty>K\left\|\mu^{-\gamma} P \phi \widetilde{U}\right\|^{2} \geq \gamma\left\|\mu^{-\gamma} \nabla_{y} \phi \widetilde{U}\right\|^{2}+\gamma^{2}\left\|\mu^{-1}\left(\mu^{-\gamma} \phi \widetilde{U}\right)_{\mu}\right\|^{2}+\gamma^{3}\left\|\mu^{-2-\gamma} \phi \widetilde{U}\right\|^{2} .
$$

We know from (7.9) that $\left\|\left[\mu\left(\mu^{2}-\nu^{2}\right)\right]^{\frac{1}{2}} \nabla_{y} \phi \widetilde{U}\right\|<\infty$ and that $\|\phi \widetilde{U}\|<\infty$. Since $\phi \widetilde{U}$ is supported in $\mu \geq|\nu|$, we have

$$
\left\|\mu^{-\frac{1}{2}}\left(\mu^{2}-\nu^{2}\right) \nabla_{y} \widetilde{U}\right\|<\infty \text { and }\left\|\mu^{-\frac{1}{2}}\left(\mu^{2}-\nu^{2}\right) \phi \widetilde{U}\right\|_{L^{2}\left([0, T] \times[0, T] ; H^{-1}\left(Y_{0}\right)\right)}<\infty .
$$

So applying (7.39) with $\gamma=\frac{1}{2}$ we obtain

$$
\left\|\mu^{-\frac{1}{2}} \nabla_{y} \phi \widetilde{U}\right\|<\infty, \quad\left\|\mu^{-\frac{5}{2}} \phi \widetilde{U}\right\|<\infty .
$$

Thus we can apply (7.39) with $\gamma=2$. Repeating this argument we obtain

$$
\mu^{-\gamma} \nabla_{y}(\phi \widetilde{U}), \quad \mu^{-1}\left(\mu^{-\gamma} \phi \widetilde{U}\right)_{t}, \quad \mu^{-\gamma-2} \phi \widetilde{U} \in L^{2}((-T, T) \times(-T, T) \times \partial M), \quad \forall \gamma>\frac{1}{10} .
$$


Equation (7.39) shows in particular that

$$
\begin{gathered}
\gamma^{3}\left\|\mu^{-\gamma-2} \widetilde{U}\right\|_{L^{2}\left((-T / 2, T / 2) \times(T, T) \times Z_{0}\right)}=\gamma^{3}\left\|\mu^{-\gamma-2} \phi \widetilde{U}\right\|_{L^{2}\left((-T / 2, T / 2) \times(T, T) \times Y_{0}\right)} \leq \\
\leq \gamma^{3}\left\|\mu^{-\gamma-2} \phi \widetilde{U}\right\|_{L^{2}\left((-T, T) \times(T, T) \times Y_{0}\right)} \leq K\left\|\mu^{-\gamma} P \phi \widetilde{U}\right\|_{L^{2}\left((-T, T) \times(\epsilon, \epsilon) \times Y_{0}\right)}
\end{gathered}
$$

Using that $\mu>\frac{1}{2} T$ on the support of $P \phi \widetilde{U}$ we arrive at

$$
\left(\frac{T}{2}\right)^{-\gamma-2} \gamma^{3}\|\widetilde{U}\|_{L^{2}\left((-T / 2, T / 2) \times(-T / 2, T / 2) \times Z_{0}\right)} \leq K\left(\frac{T}{2}\right)^{-\gamma}\|P \phi \widetilde{U}\|_{L^{2}\left((-T, T) \times(-T, T) \times Y_{0}\right)} .
$$

So in particular

$$
\gamma^{3}\|\widetilde{U}\|_{L^{2}\left((-T / 2, T / 2) \times(-T / 2, T / 2) \times Z_{0}\right)} \leq K\left(\frac{T}{2}\right)^{2}\|P \phi \widetilde{U}\|_{L^{2}\left((-T, T) \times(-T, T) \times Y_{0}\right)} .
$$

By (7.9) and the definition of $P$, the right hand side of (7.40) is finite. Then letting $\gamma \rightarrow \infty$ gives $\widetilde{U}=0$ in $(-T / 2, T / 2) \times(-T / 2, T / 2) \times Z_{0}$. Since $\partial X$ is compact, this argument can be applied to a partition of unity consisting of finitely many functions to deduce that there exists $\epsilon>0$ such that $\widetilde{U}=0$ in $(-\epsilon, \epsilon) \times(-\epsilon, \epsilon) \times \partial X$. In particular $f(x, y)=0$ if $x<\sqrt{\epsilon}$. This ends the proof of the Lemma.

To conclude the proof of Theorem 7.1 we need

Lemma 7.3. If $f \in L_{\mathrm{comp}}^{2}(\stackrel{\circ}{X})$ and $\mathcal{R}_{+}(0, f)$ is supported in $s>s_{0}$, with $s_{0}<<0$, then $f$ is supported in $x>e^{s_{0}}$.

Proof. Let $\phi \in C_{0}^{\infty}(\mathbb{R})$ be even, supported in $(-1,1)$, and $\phi_{\epsilon}(s)=\epsilon^{-n} \phi(s / \epsilon)$. Then $\phi_{\epsilon} * \mathcal{R}_{+}(0, f)$ is supported in $s>s_{0}-\epsilon$. Let $\psi_{1} \in \mathcal{S}(\mathbb{R})$ and $\psi_{1, \epsilon} \in \mathcal{S}(\mathbb{R})$ be the functions obtained from $\phi$ and $\phi_{\epsilon}$ by $(7.2)$. It follows from Lemma 7.2 that $\psi_{1, \epsilon}\left(\Delta-\frac{n^{2}}{4}\right) f$ is compactly supported. If we prove that $\psi_{1, \epsilon}\left(\Delta-\frac{n^{2}}{4}\right) f$ is supported in $x \geq e^{s_{0}-\epsilon}$ then by letting $\epsilon \rightarrow 0$ we have proved Lemma [7.3. So we may assume that $f \in C_{0}^{\infty}(\stackrel{\circ}{X})$, and let us say that $f(x, y)=0$ for $x<x_{0}$. Then (4.3), which is due to the finite speed of propagation, gives that

$$
V\left(x^{\prime}, t^{\prime}, y\right)=0 \quad \text { if } \quad t^{\prime}<\sqrt{x_{0}} .
$$

Moreover we know from Theorem[2.1 that $V$ is smooth up to $x^{\prime}=0$. Since by assumption $V\left(0, t^{\prime}, y\right)=0$ for $t^{\prime}<t_{0}=\exp \left(\frac{s_{0}}{2}\right)$, then, as proved above, $V$ extends to a smooth solution to (4.8) for $x^{\prime}<0$ which vanishes in $\left\{x^{\prime}<0, \quad t^{\prime}<t_{0}\right\}$.

The principal symbol of $P^{\prime}$ is given by (7.5), so it is easily seen that the level surfaces of $\left\{\phi=-t^{\prime}-x^{\prime}\right\}$ are not characteristic for $P^{\prime}$. The Hamiltonian of $p^{\prime}, H_{p^{\prime}}$, was computed in (7.6). Then

$$
\begin{gathered}
p^{\prime}\left(0, t^{\prime}, y, \xi, \tau, \eta\right)=-\xi \tau, \quad\left(H_{p^{\prime}} \phi\right)\left(0, t^{\prime}, y, \xi, \tau, \eta\right)=\tau+\xi, \quad \text { and } \\
\left(H_{p^{\prime}}^{2} \phi\right)\left(0, t^{\prime}, y, \xi, \tau, \eta\right)=t^{\prime} h(0, y, \eta),
\end{gathered}
$$

and therefore, for $t^{\prime}>0$,

$$
\text { if } \begin{gathered}
p^{\prime}\left(0, t^{\prime}, y, \xi, \tau, \eta\right)=\left(H_{p^{\prime}} \phi\right)\left(0, t^{\prime}, y, \xi, \tau, \eta\right)=0, \quad \text { with }(\xi, \tau, \eta) \neq 0 \text { then } \\
\left(H_{p^{\prime}}^{2} \phi\right)\left(0, t^{\prime}, y, \xi, \tau, \eta\right)>0 .
\end{gathered}
$$

This, according to section 28.4 of [26], implies that the level surfaces of $\phi$ are strongly pseudo-convex with respect to $P^{\prime}$, as long as $t^{\prime}>0$. So applying Hörmander's theorem, Theorem 28.3.4 of [26], to (4.8]) and the surface $\left\{\phi=-\sqrt{x_{0}}\right\}$ we find that $V\left(x^{\prime}, t^{\prime}, y\right)=0$ near $\left(0, \sqrt{x_{0}}, y\right)$. Now we can repeat this argument to show that $V=0$ in a neighborhood of the segment $\left[0, t_{0}\right]$. And we can proceed in the same way to conclude that in fact $V=0$ in $\left\{\left(x^{\prime}, t^{\prime}, y\right): 0<x^{\prime}<\sqrt{x_{0}}, 0<t^{\prime}<t_{0}\right\}$. By the symmetry of $V$, it follows 

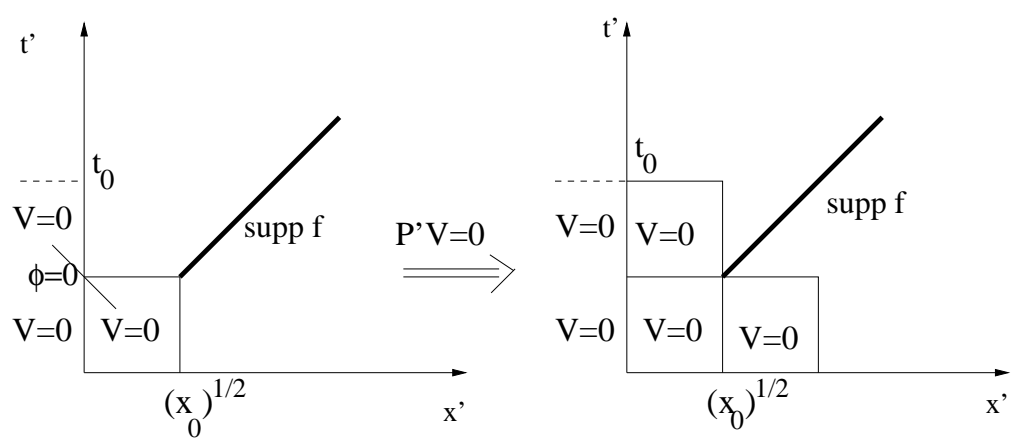

Figure 4. Unique continuation for $V$. Away from $x^{\prime}=t^{\prime}=0$, the level surfaces of $\phi$ are strongly pseudo-convex.

that $V=0$ in $\left\{\left(x^{\prime}, t^{\prime}, y\right): 0<t^{\prime}<\sqrt{x_{0}}, 0<x^{\prime}<t_{0}\right\}$, see figure 4 This is the point where we use that the initial data is $(0, f)$.

Now going back to the variables $(x, t, y)$ which are given by $x=x^{\prime} t^{\prime}, t=\log t^{\prime}-\log x^{\prime}$, this implies that the solution $u(x, t, y)$ of (2.5) with initial data $(0, f)$ satisfies

$$
u(x, t, y)=0 \text { for }\left\{(x, t, y): 0<x<x_{0}, \log x_{0}-s_{0}<t<s_{0}-\log x_{0}\right\} .
$$

One particular case of Tataru's theorem [49, see also [27, 46, 47, 50, states that if $u(t, z)$ is in $H_{\text {loc }}^{1}$ and satisfies

$$
\begin{gathered}
\left(D_{t}^{2}-\Delta-\frac{n^{2}}{4}\right) u(t, z)=0 \\
u(t, z)=0 \quad-T<t<T, \text { and } d\left(z, z_{0}\right)<\delta, \quad \delta>0,
\end{gathered}
$$

where $d\left(z, z_{0}\right)$ is the distance between $z$ and $z_{0}$ with respect to the metric $g$, then

$$
u(t, z)=0 \text { for }|t|+d\left(z, z_{0}\right)<T .
$$

We then deduce from (7.42) and (7.43) that $u(0, z)=D_{t} u(0, z)=0$ on the set of points $z=(x, y)$ at a distance less than $s_{0}-\log x_{0}$ from the surface $\left\{x=x_{0}\right\}$. In these coordinates the distance is given by $\log x-\log x_{0}$. So $f(x, y)=D_{t} u(0, x, y)=0$ for $\log x<s_{0}$. This proves Lemma 7.3

\section{The Inverse Problem}

We consider the inverse problem of determining the manifold and the metric from the scattering matrix $A(\lambda)$, at all energies $\lambda \in \mathbb{R} \backslash 0$. We remark that, as $A(\lambda)$ has a meromorphic continuation to $\mathbb{C} \backslash \frac{i}{2} \mathbb{N}$, see [28, this assumption implies that $A_{1}(\lambda)=A_{2}(\lambda)$ for $\lambda \in \mathbb{C} \backslash \frac{i}{2} \mathbb{N}$.

We are fixing a defining function $x$ of $\partial X$ for which (2.4) holds near $M$ and using it in (2.3) to define $A(\lambda)$. According to Lemma 2.1 of [15], $x$ is uniquely determined near $M$ by the choice of the conformal representative $h_{0}=\left.x^{2} g\right|_{\partial X}$. So to define the scattering matrix we fix a conformal representative $h_{0}$. On the other hand, we recall from Theorem 1.1 and Corollary 1.1 of 28 that $A(\lambda)$, for $\lambda$ fixed, determines the conformal representative $h_{0}$. So if we say that two asymptotically hyperbolic metrics $g_{1}$ and $g_{2}$ on $X$ have the same scattering matrix, as defined by (2.3), it is implicit that we are fixing the same conformal representative for $\left.x_{j}^{2} g_{j}\right|_{\partial X}, j=1,2$.

We will prove

Theorem 8.1. Let $\left(X_{1}, g_{1}\right)$ and $\left(X_{2}, g_{2}\right)$ be asymptotically hyperbolic manifolds which have the same boundary $\partial X_{1}=\partial X_{2}=M$. Let $x_{j} \in C^{\infty}\left(X_{j}\right)$ be a defining function of $M=\partial X_{j}, j=1,2$, for which 
(2.4) holds, and let $A_{j}(\lambda), j=1,2, \lambda \in \mathbb{R} \backslash 0$, be the corresponding scattering matrices defined in (2.3) in terms of $x_{j}$. Suppose that $A_{1}(\lambda)=A_{2}(\lambda)$ for every $\lambda \in \mathbb{R} \backslash 0$. Then there exists a diffeomorphism $\Psi: X_{1} \longrightarrow X_{2}$, smooth up to $M$, such that

$$
\Psi=\mathrm{Id} \text { at } M \text { and } \Psi^{*} g_{2}=g_{1} .
$$

As mentioned in the introduction, the proof is an application of the control method of Belishev 4, see also [5, 31, 29, 30. We will also use a result, which is an application of this method, and is due to Katchalov and Kurylev [29, 30]

First we construct a diffeomorphism between neighborhoods of the boundary that realizes (8.1) and later show that it can be extended to a diffeomorphism between the two manifolds.

We recall that Lemma 2.1 of [15] states that fixed a conformal representative $h_{0}$ there exists a unique defining function $x_{i}$, in a neighborhood of $M$ for which (2.4) holds. However $x_{i}$ can be continued to $X_{i}$, although not uniquely. As explained in the paragraph before Theorem 8.1] it is implicit that we are fixing a conformal representative

$$
h_{0}=\left.x_{1}^{2} g_{1}\right|_{M}=\left.x_{2}^{2} g_{2}\right|_{M} .
$$

Note that, as observed in the paragraph following the proof Lemma 2.1 of [15], for $\epsilon>0$ small, a defining function $x_{i}$ for which (2.4) holds near $M$ gives an identification of $[0, \epsilon) \times \partial M$ with a collar neighborhood $U_{i, \epsilon} \subset \overline{X_{i}}$ of $M$ by

$$
\Psi_{i, \epsilon}:[0, \epsilon) \times M \longrightarrow U_{i, \epsilon},
$$

where $\Psi_{i, \epsilon}(x, y)$ is the point obtained by flowing the integral curve of $\nabla_{x_{i}^{2} g_{i}} x_{i}$ emanating from $y$ by $x$ units of time. So $x$ is the arc-length along the geodesics normal to the boundary $M$ with respect to the metrics $x_{i}^{2} g_{i}$, or the distance from the point $(x, y)$ to $M$ with respect to this metric. We can pick $\epsilon$ small such that these maps are diffeomorphisms. Then $x$ is a smooth defining function of $M$,

$$
\begin{gathered}
x:[0, \epsilon) \times M \longrightarrow \mathbb{R}, \quad x=x_{i}+o\left(x_{i}\right), \quad \text { and } \\
g_{j}=\frac{d x^{2}}{x^{2}}+\frac{h_{j}}{x^{2}}, \quad j=1,2 .
\end{gathered}
$$

In fact for each $y \in M$ there exists $\epsilon_{i}(y)>0$ such that for $x<\epsilon_{i}(y)$ the distance between $(x, y)$ and $M$, with respect to the metric $x_{i}^{2} g_{i}$, is equal to $x$. So $\Psi_{i, \epsilon}$ extends to a diffeomorphism

$$
\Psi_{i}:\left[0, \epsilon_{i}(y)\right) \times M \longrightarrow X_{i} \backslash \Gamma_{i},
$$

where $\Gamma_{i}$ is the cut-locus of $X_{i}$ with respect to $x_{i}^{2} g_{i}$. It is known that the set $\Gamma_{i}$ is a closed subset of measure zero and $\Gamma_{i} \cap M=\emptyset$. The number $\epsilon$ in (8.4) is less than the smallest of the distances between $\Gamma_{i}$ and $M$.

We will fix one such function throughout this section and we will prove

Proposition 8.2. Let $\left(X_{1}, g_{1}\right)$ and $\left(X_{2}, g_{2}\right)$ be asymptotically hyperbolic manifolds satisfying the hypotheses of Theorem 8.1. Then there exists $\epsilon>0$ such that in the product decomposition $X \sim[0, \epsilon) \times \partial X$ where (8.4) holds, $h_{1}=h_{2}$.

The fact that the metrics are equal in these coordinates imply that

$$
\Psi_{1, \epsilon}^{*}\left(\left.g_{1}\right|_{U_{1, \epsilon}}\right)=\Psi_{2, \epsilon}^{*}\left(\left.g_{2}\right|_{U_{2, \epsilon}}\right)
$$

with $\Psi_{i, \epsilon}$ defined in (8.3), and therefore

$$
\left[\Psi_{2, \epsilon} \circ \Psi_{1, \epsilon}^{-1}\right]^{*}\left(\left.g_{2}\right|_{U_{2, \epsilon}}\right)=\left.g_{1}\right|_{U_{1, \epsilon}} .
$$

This gives a diffeomorphism between neighborhoods of the boundary satisfying (8.1). 
8.1. Preliminaries. Here we define some spaces that will be useful in the proof of Proposition 8.2

Notice that if $u$ is a solution of (2.5) with initial data $(0, f)$, then $u(t, z)=-u(-t, z), t \in \mathbb{R}$. Similarly, if $u$ is a solution of (2.5) with initial data $(f, 0)$, then $u(t, z)=u(-t, z), t \in \mathbb{R}$. This implies that

$$
\mathcal{R}_{+}(0, f)(-s, y)=\mathcal{R}_{-}(0, f)(s, y), \quad \text { and } \quad \mathcal{R}_{+}(f, 0)(-s, y)=-\mathcal{R}_{-}(f, 0)(s, y) .
$$

Proposition 8.3. Let $(X, g)$ be an asymptotically hyperbolic manifold and let $x$ be a defining function of $\partial X$ for which (2.4) holds. For $F \in L^{2}(\mathbb{R} \times \partial X)$, let $F^{*}(s, y)=F(-s, y)$. Let $\mathcal{R}_{ \pm}$denote the radiation fields defined with respect to $x$, and let $\mathcal{S}=\mathcal{R}_{+} \mathcal{R}_{-}^{-1}$ be the scattering operator. Let

$$
\mathcal{M}^{f}=\left\{F \in L^{2}(\mathbb{R} \times \partial X): \quad F=\mathcal{S} F^{*}\right\} \quad \text { and } \quad \mathcal{M}^{b}=\left\{F \in L^{2}(\mathbb{R} \times \partial X): \quad F^{*}=\mathcal{S} F\right\},
$$

where $f$ and $b$ stand for forward and backward. Then

$$
\mathcal{M}^{f}=\left\{\mathcal{R}_{+}(0, f): \quad f \in L_{\mathrm{ac}}^{2}(X)\right\} \quad \text { and } \quad \mathcal{M}^{b}=\left\{\mathcal{R}_{-}(0, f): \quad f \in L_{\mathrm{ac}}^{2}(X)\right\}
$$

Proof. We will prove the first equality. The proof of the second one is identical. If $F=\mathcal{R}_{+}(0, f)$, then according to (8.6), $F^{*}=\mathcal{R}_{-}(0, f)$, and so

$$
\mathcal{S} F^{*}=\mathcal{R}_{+} \mathcal{R}_{-}^{-1} F^{*}=\mathcal{R}_{+}(0, f)=F .
$$

Notice also that if $F=\mathcal{R}_{+}(g, 0)$, then according to [8.6),$F^{*}=-\mathcal{R}_{-}(g, 0)$, and so

$$
\mathcal{S} F^{*}=\mathcal{R}_{+} \mathcal{R}_{-}^{-1} F^{*}=-\mathcal{R}_{+}(g, 0)=-F .
$$

Conversely, let $F \in L^{2}(\mathbb{R} \times \partial X)$. We know from Theorem 5.1 that there exists $\left(f_{1}, f_{2}\right) \in E_{\text {ac }}$ such that $F=\mathcal{R}_{+}\left(f_{1}, f_{2}\right)$. Let $F_{1}=\mathcal{R}_{+}\left(f_{1}, 0\right)$ and $F_{2}=\mathcal{R}_{+}\left(0, f_{2}\right)$. If $F=\mathcal{S} F^{*}$, then

$$
F=F_{1}+F_{2}=S F_{1}^{*}+\mathcal{S} F_{2}^{*} \text {. }
$$

In view of the discussion above, $\mathcal{S} F_{2}^{*}=F_{2}$ and $\mathcal{S} F_{1}^{*}=-F_{1}$. So $F_{1}+F_{2}=-F_{1}+F_{2}$, and thus $F_{1}=0$. By uniqueness, $f_{1}=0$.

As ranges of bounded operators, $\mathcal{M}^{f}$ and $\mathcal{M}^{b}$ are closed. By Proposition 8.3 and Theorem 5.2 they are also defined in terms of the scattering matrix and the space $L^{2}(\mathbb{R} \times \partial X)$, which depends on the volume element of the metric $h(0, y, d y)$. However, as discussed above, the scattering matrix determines the tensor $h(0, x, d y)$, so $\mathcal{M}^{f}$ and $\mathcal{M}^{b}$ are determined by $A(\lambda), \lambda \in \mathbb{R} \backslash 0$. Thus we have

Corollary 8.4. Let $(X, g)$ be an asymptotically hyperbolic manifold. Let $x$ be a defining function of $\partial X$ for which (2.4) holds and let $\mathcal{R}_{ \pm}$denote the radiation fields defined with respect to $x$. Then the spaces $\mathcal{M}^{f}$ and $\mathcal{M}^{b}$ defined in (8.7) are closed subspaces of $L^{2}(\mathbb{R} \times \partial X)$, and so are Hilbert spaces with the inherited norm. Moreover, $\mathcal{M}^{f}$ and $\mathcal{M}^{b}$ are determined by the scattering matrix $A(\lambda)$, for all $\lambda \in \mathbb{R} \backslash 0$.

We will need

Lemma 8.5. Let $(X, g)$ be an asymptotically hyperbolic manifold. Let $\mathcal{M}^{f}$ and $\mathcal{M}^{b}$ be defined in (8.7). For $x_{1} \in(0, \epsilon)$, we have

$$
\begin{aligned}
& \mathcal{M}^{f}\left(x_{1}\right) \stackrel{\text { def }}{=}\left\{F \in \mathcal{M}^{f}, \quad F(s, y)=0 \quad \text { if } \quad s<\log x_{1}\right\}=\left\{\mathcal{R}_{+}(0, f): \quad f \in L_{\mathrm{ac}}^{2}(X), \quad f=0 \quad \text { if } \quad x<x_{1}\right\}, \\
& \mathcal{M}^{b}\left(x_{1}\right) \stackrel{\text { def }}{=}\left\{F \in \mathcal{M}^{b}, \quad F(s, y)=0 \quad \text { if } \quad s>-\log x_{1}\right\}=\left\{\mathcal{R}_{-}(0, f): \quad f \in L_{\mathrm{ac}}^{2}(X), \quad f=0 \quad \text { if } \quad x<x_{1}\right\} .
\end{aligned}
$$

Proof. Finite speed of propagation guarantees that if $f \in L_{\mathrm{ac}}^{2}(X)$ and $f=0$ in $\left\{x<x_{1}\right\}$, then $F=$ $\mathcal{R}_{+}(0, f) \in \mathcal{M}^{f}\left(x_{1}\right)$ and $F=\mathcal{R}_{-}(0, f) \in \mathcal{M}^{b}\left(x_{1}\right)$. On the other hand, if $F \in \mathcal{M}^{f}\left(x_{1}\right)$, in particular $F \in \mathcal{M}^{f}$, and thus by Proposition 8.3 $F=\mathcal{R}_{+}(0, f)$ with $f \in L_{\text {ac }}^{2}(X)$. Since $F(s, y)=0$ if $s<\log x_{1}$, it follows from Theorem 7.1 that $f=0$ in $x<x_{1}$. 
If $F \in \mathcal{M}^{b}\left(x_{1}\right)$, then $F=\mathcal{R}_{-}(0, g)$. So $F^{*}=\mathcal{R}_{+}(0, g)$ and $F^{*}=0$ for $s<\log x_{1}$. Therefore Theorem 7.1 gives that $g=0$ in $x<x_{1}$.

We emphasize that the proof of Lemma 8.5 required the full power Theorem 7.1 and this is where the support theorem enters in the study of the inverse problem.

In what follows, for $F \in \mathcal{M}^{f}$, or $F \in \mathcal{M}^{b}$, we will use, by an abuse of notation, $\mathcal{R}_{ \pm}^{-1} F=f$, and $\mathcal{R}_{ \pm} f=F$ instead of $\mathcal{R}_{ \pm}^{-1} F=(0, f)$ and $\mathcal{R}_{ \pm}(0, f)=F$, respectively.

8.2. The case of no eigenvalues. To better explain our methods, we will first consider the case where the manifolds have no eigenvalues. In this particular case, Proposition 8.2 is an easy consequence of

Proposition 8.6. Let $g_{1}$ and $g_{2}$ be asymptotically hyperbolic metrics satisfying the hypotheses of Theorem 8.1. Moreover assume that $\Delta_{g_{i}}, i=1,2$, have no eigenvalues. Let $\mathcal{R}_{j, \pm}, j=1,2$, denote the corresponding forward or backward radiation fields defined in coordinates in which (8.4) holds. Then there exists $\epsilon>0$ such that

$$
\begin{aligned}
& \left|h_{1}\right|^{\frac{1}{4}}(x, y) \mathcal{R}_{1,-}^{-1} F(x, y)=\left|h_{2}\right|^{\frac{1}{4}}(x, y) \mathcal{R}_{2,-}^{-1} F(x, y), \quad(x, y) \in(0, \epsilon) \times M, \quad \forall F \in \mathcal{M}^{b}, \\
& \left|h_{1}\right|^{\frac{1}{4}}(x, y) \mathcal{R}_{1,+}^{-1} F(x, y)=\left|h_{2}\right|^{\frac{1}{4}}(x, y) \mathcal{R}_{2,+}^{-1} F(x, y), \quad(x, y) \in(0, \epsilon) \times M, \quad \forall F \in \mathcal{M}^{f} .
\end{aligned}
$$

Indeed, suppose Proposition 8.6 has been proved. We will prove Proposition 8.2

Proof. Let $x$ be such that (8.4) holds. We know that for any $F \in \mathcal{M}^{b}$,

$$
\mathcal{R}_{j,-}^{-1}\left(\frac{\partial^{2}}{\partial s^{2}} F\right)=\left(\Delta_{g_{j}}-\frac{n^{2}}{4}\right) \mathcal{R}_{j,-}^{-1} F
$$

So the first equation in (8.10) implies that for $(x, y) \in(0, \epsilon) \times M$, and any $F \in \mathcal{M}^{b}$,

$$
\begin{gathered}
\left|h_{1}\right|^{\frac{1}{4}}(x, y) \mathcal{R}_{1,-}^{-1} F(x, y)=\left|h_{2}\right|^{\frac{1}{4}}(x, y) \mathcal{R}_{2,-}^{-1} F(x, y), \text { and } \\
\left|h_{1}\right|^{\frac{1}{4}}(x, y)\left(\Delta_{g_{1}}-\frac{n^{2}}{4}\right) \mathcal{R}_{1,-}^{-1} F(x, y)=\left|h_{2}\right|^{\frac{1}{4}}(x, y)\left(\Delta_{g_{2}}-\frac{n^{2}}{4}\right) \mathcal{R}_{2,-}^{-1} F(x, y) .
\end{gathered}
$$

Set $\mathcal{R}_{1,-}^{-1} F=f$. Since $F$ is arbitrary and the metrics have no eigenvalues, equations (8.12) give, in particular,

$$
\begin{gathered}
\left|h_{1}\right|^{\frac{1}{4}}(x, y)\left(\Delta_{g_{1}}-\frac{n^{2}}{4}\right) f(x, y)=\left|h_{2}\right|^{\frac{1}{4}}(x, y)\left(\Delta_{g_{2}}-\frac{n^{2}}{4}\right) \frac{\left|h_{1}\right|^{\frac{1}{4}}(x, y)}{\left|h_{2}\right|^{\frac{1}{4}}(x, y)} f(x, y), \\
\forall f \in C_{0}^{\infty}((0, \epsilon) \times M) .
\end{gathered}
$$

Therefore the operators on both sides of (8.13) are equal. In particular the coefficients of the principal parts of $\Delta_{g_{1}}$ are equal to those of $\Delta_{g_{2}}$, and hence the tensors $h_{1}$ and $h_{2}$ from (8.4) are equal. This proves Proposition 8.2

We begin the proof of Proposition 8.6 with

Lemma 8.7. Let $(X, g)$ be an asymptotically hyperbolic manifold such that $\Delta_{g}$ has no eigenvalues. Let $x$ be such that (2.4) holds in $(0, \epsilon) \times M$. For $x_{1} \in(0, \epsilon)$, let $\mathcal{P}_{x_{1}}^{b}$ denote the orthogonal projector

$$
\mathcal{P}_{x_{1}}^{b}: \mathcal{M}^{b} \longrightarrow \mathcal{M}^{b}\left(x_{1}\right),
$$

and let $\chi_{x_{1}}$ be the characteristic function of the set $\left\{x \geq x_{1}\right\}$. Then for every $f \in L_{\mathrm{ac}}^{2}(X)=L^{2}(X)$,

$$
\mathcal{P}_{x_{1}}^{b} \mathcal{R}_{-}(0, f)=\mathcal{R}_{-}\left(0, \chi_{x_{1}} f\right) \in \mathcal{M}^{b}\left(x_{1}\right) .
$$


Proof. Since $\mathcal{P}_{x_{1}}^{b}$ is a projector, then for all $G \in \mathcal{M}^{b}\left(x_{1}\right)$

$$
\left\langle\mathcal{P}_{x_{1}}^{b} \mathcal{R}_{-}(0, f), G\right\rangle_{L^{2}(\mathbb{R} \times \partial X)}=\left\langle\mathcal{R}_{-}(0, f), G\right\rangle_{L^{2}(\mathbb{R} \times \partial X)} .
$$

In particular, since $L_{\mathrm{ac}}^{2}(X)=L^{2}(X)$, then for all $g \in C_{0}^{\infty}(\stackrel{\circ}{X})$ supported in $\left\{x>x_{1}\right\}$,

$$
\left\langle\mathcal{P}_{x_{1}}^{b} \mathcal{R}_{-}(0, f), \mathcal{R}_{-}(0, g)\right\rangle_{L^{2}(\mathbb{R} \times \partial X)}=\langle f, g\rangle_{L^{2}(X)} .
$$

On the other hand, by Lemma 8.5 there exists $f_{x_{1}} \in L^{2}(X)$ supported in $\left\{x \geq x_{1}\right\}$ such that $\mathcal{P}_{x_{1}}^{b} \mathcal{R}_{-}(0, f)=\mathcal{R}_{-}\left(0, f_{x_{1}}\right)$. Therefore, for all $g \in C_{0}^{\infty}(\stackrel{\circ}{X})$ supported in $\left\{x>x_{1}\right\}$,

$$
\left\langle\mathcal{P}_{x_{1}}^{b} \mathcal{R}_{-}(0, f), \mathcal{R}_{-}(0, g)\right\rangle_{L^{2}(\mathbb{R} \times \partial X)}=\left\langle\mathcal{R}_{-}\left(0, f_{x_{1}}\right), \mathcal{R}_{-}(0, g)\right\rangle_{L^{2}(\mathbb{R} \times \partial X)}=\left\langle f_{x_{1}}, g\right\rangle_{L^{2}(X)} .
$$

Thus

$$
\left\langle f_{x_{1}}, g\right\rangle_{L^{2}(X)}=\langle f, g\rangle_{L^{2}(X)}, \quad \forall g \in L^{2}(X) \text { supported in }\left\{x \geq x_{1}\right\},
$$

and so $f_{x_{1}}=\chi_{x_{1}} f$.

This ends the proof of the Proposition.

Remark 8.8. Since the spaces $\mathcal{M}^{f}, \mathcal{M}^{b}, \mathcal{M}^{f}\left(x_{1}\right)$ and $\mathcal{M}^{b}\left(x_{1}\right)$ are determined by the scattering matrix, so are the projectors $\mathcal{P}_{x_{1}}^{f}$ and $\mathcal{P}_{x_{1}}^{b}$.

We use this and properties of propagation of singularities for solutions to the wave equation to prove

Lemma 8.9. Let $(X, g)$ be an asymptotically hyperbolic manifold which has no eigenvalues. Let $x$ be a boundary defining function such that (2.4) holds. Let $\mathcal{R}_{ \pm}$be the radiation fields with respect to $x$. Then, there exists $\epsilon>0$, such that for any $x_{1} \in(0, \epsilon / 4)$, any $F \in \mathcal{M}^{b}$, any $\phi \in C_{0}^{\infty}(\mathbb{R})$ even, and the corresponding $\psi_{1}$ be defined by (7.2),

$$
\begin{aligned}
& \mathcal{R}_{+} \mathcal{R}_{-}^{-1}\left(\mathcal{P}_{x_{1}}^{b} G\right)(s, y)=\frac{1}{2} x_{1}^{-\frac{n}{2}} w\left(x_{1}, y\right) \frac{|h|^{\frac{1}{4}}\left(x_{1}, y\right)}{|h|^{\frac{1}{4}}(0, y)}\left(s-\log x_{1}\right)_{+}^{0}+\text { smoother terms, } \\
& \text { if } s<\log x_{1}+\log 4, \quad \text { where } G=\phi * F, \quad \text { and } \quad w=\mathcal{R}_{-}^{-1} G=\psi_{1}\left(\Delta_{g}-\frac{n^{2}}{4}\right) \mathcal{R}_{-}^{-1} F .
\end{aligned}
$$

Notice that by (7.3) $G \in \mathcal{M}^{b}$, and that, according to Remark 8.8, the left hand side of (8.14) is determined by the scattering matrix of $g$.

Proof. We choose $\epsilon>0$ so that the maps $\Psi_{j, \epsilon}$ defined by (8.3) are diffeomorphisms. That is, the distance from $\Gamma$, the cut-locus, to $M$ is greater than $\epsilon$, and take $x_{1}<\frac{\epsilon}{4}$. From Lemma 8.7 we know that

$$
\left(0, \chi_{x_{1}} w\right)=\mathcal{R}_{-}^{-1}\left(\mathcal{P}_{x_{1}}^{b} G\right)
$$

with $\chi_{x_{1}}$ being the characteristic function of the set $\left\{x \geq x_{1}\right\}$, which is the set of points whose distance to $M$ is greater than or equal to $x_{1}$, and hence

$$
\mathcal{R}_{+}\left(0, \chi_{x_{1}} w\right)=\mathcal{R}_{+} \mathcal{R}_{-}^{-1}\left(\mathcal{P}_{x_{1}}^{b} G\right) .
$$

So we want to analyze $\mathcal{R}_{+}\left(0, \chi_{x_{1}} w\right)$. Recall from the definition of the forward radiation field that this amounts to finding the solution $u$ to (2.5) with initial data $\left(0, \chi_{x_{1}} w\right)$, then taking $v(s, x, y)=$ $x^{-\frac{n}{2}} u(s-\log x, x, y)$ and restricting $\frac{\partial v}{\partial s}$ to $x=0$. We remark that, although this is the definition of the forward radiation field for $C_{0}^{\infty}(\stackrel{\circ}{X})$ data, it follows from the discussion in section 7 that this also holds for initial data in $L_{\text {ac }}^{2}(X)=L^{2}(X)$, see equations (7.13) and (7.16). We are concerned with the restriction of $\frac{\partial v}{\partial s}$ to $x=0$, so we will only consider the behavior of $v$ for $s>\log x$.

The initial data $\chi_{x_{1}} w$ has a conormal singularity at $S=\left\{s=\log x, x=x_{1}\right\}$, therefore the wavefront set of $v$ will be contained in the flow-out of $N^{*} S \cap \Sigma$, where $\Sigma$ is the characteristic variety of $P$, which 


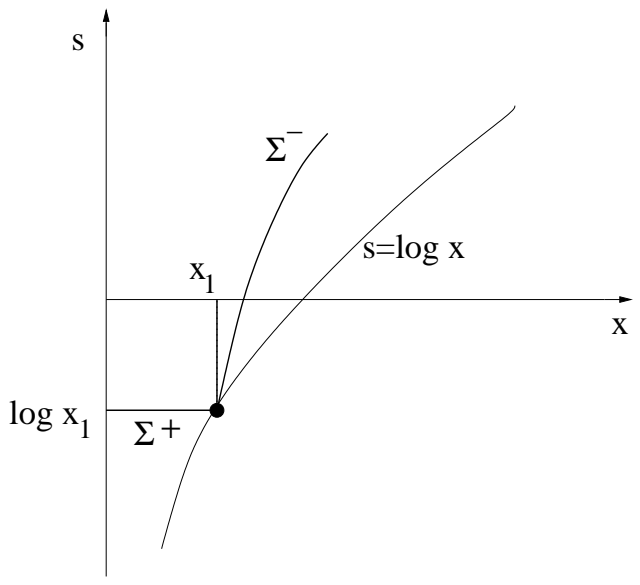

FiguRE 5. The singularities of $v$ in $s>\log x$.

is defined in 4.5. The principal symbol of $P$ is $p=-2 \xi \sigma-x \xi^{2}-x h(x, y, \eta), \sigma$ is the dual to $s$. So the null bicharacteristics satisfy

$$
\begin{gathered}
\dot{x}=-2 \sigma-2 x \xi, \quad \dot{s}=-2 \xi, \quad \dot{y}=-x \frac{\partial h}{\partial \eta}, \quad \dot{\xi}=\xi^{2}+h+x \frac{\partial h}{\partial x}, \quad \dot{\eta}=x \frac{\partial h}{\partial y}, \quad \dot{\sigma}=0, \\
x(0)=x_{1}, \quad s(0)=\log x_{1}, \quad y(0)=y_{0}, \quad \xi(0)=\xi_{0}, \quad \eta_{0}=\eta_{0}, \quad \sigma(0)=\sigma_{0}, \quad 2 \sigma \xi+x \xi^{2}+x h(x, y, \eta)=0 .
\end{gathered}
$$

Note that since $p=0$ is satisfied for the solutions to this system, and $\sigma=\sigma_{0} \neq 0$, we must have

$$
\xi=\frac{1}{x}\left(-\sigma_{0} \pm\left(\sigma_{0}^{2}-x^{2} h\right)^{\frac{1}{2}}\right) .
$$

Since we are concerned with the forward singularities, we must have $\sigma_{0}>0$. Then it follows that $\xi \leq 0$, and thus $s$ is non-decreasing.

We analyze the singularities that start over $N^{*} S=\left\{x=x_{1}, s=\log x_{1}, \eta_{0}=0\right\}$. Using $x$ as a parameter, we find two families of curves

$$
\begin{aligned}
& s=\log x_{1}, y=y_{0}, \quad \xi=0, \quad \eta=\eta_{0}, \sigma=\sigma_{0} \quad \text { if } \xi_{0}=0, \quad \text { and } \\
& s=2 \log x-\log x_{1}, y=y_{0}, \quad \eta=\eta_{0}, \quad \sigma=\sigma_{0}, \quad \xi=\frac{\xi_{0} x_{1}}{x}, \quad \text { if } \xi_{0}<0, \quad \text { and } 2 \sigma_{0}+x_{1} \xi_{0}=0 .
\end{aligned}
$$

These curves make up the two characteristic surfaces of $P$ emanating from $\left\{s=\log x, x=x_{1}\right\}$, which are $\Sigma^{+}=\left\{s=\log x_{1}\right\}$ and $\Sigma^{-}=\left\{s=2 \log x-\log x_{1}\right\}$. So, in $s>\log x, v$ is singular along $\Sigma^{+}$and $\Sigma^{-}$. See figure 5 .

A bicharacteristic in $\Sigma^{+}$, let us say, starting over $\left(x_{1}, y_{0}\right)$ will hit $\mathbb{R} \times M$ at $\left(\log x_{1}, y_{0}\right)$, see figure 5 . On the other hand, since $s>\log x$, note that $\Sigma^{-}$consists of null bicharacteristics which go into the interior of $X$, so they might also intersect the boundary. They could, however, become trapped in the interior and not reach $M$. Take one such curve, $\gamma$, starting over $\left(x_{1}, y\right), y \in M$, which intersects the boundary at another point $y^{\prime} \in M, y^{\prime} \neq y$. The projection of $\gamma$ onto $X$ is a geodesic with respect to the metric $x^{2} g$, which connects the points $\left(x_{1}, y\right)$ and $y^{\prime} \in M$ and which is orthogonal to $\left\{x=x_{1}\right\}$ at $\left(x_{1}, y\right)$. To reach the point $y^{\prime}$ this geodesic has to pass through the cut-locus $\Gamma$ and again reach the surface $\left\{x=x_{1}\right\}$. Since $\epsilon$ is less than the distance from $\Gamma$ to $M$ and $x_{1}<\epsilon / 4$, and along $\gamma$, for $x<\epsilon, t=s-\log x=\log x-\log x_{1}$, the geodesic reaches $\Gamma$ for $t>\log \epsilon-\log (\epsilon / 4)=\log 4$. Thus it reaches $\left\{x=x_{1}\right\}$ for $t=T>\log 4$. Now we analyze the flow of $H_{p}$ starting at the point $q$ where $\gamma$ intersects $\left\{x=x_{1}\right\}$. The surface $t=T$ becomes $s=T+\log x$ and we think of this singularity as starting at $\left\{s=T+\log x, \quad x=x_{1}\right\}$. But, as 
observed above, $s$ is increasing along the part of $\gamma$ connecting $q$ to a point over $M$. Then this singularity will hit the boundary at $y^{\prime}$ for $s>T+\log x_{1}>\log 4+\log x_{1}$. Hence the singularity of $\mathcal{R}_{+}\left(0, \chi_{x_{1}} w\right)$ at $s=\log x_{1}$ comes only from $\Sigma^{+}$, and these can be computed explicitly. Moreover $\mathcal{R}_{+}\left(0, \chi_{x_{1}} w\right)$ is supported in $s \geq \log x_{1}$ and

$$
\mathcal{R}_{+}\left(0, \chi_{x_{1}} w\right) \in C^{\infty}\left(\left(\log x_{1}, \log x_{1}+\log 4\right) \times M\right) .
$$

As commonly done in this type of problem, we will find a conormal expansion for $v$ along $\left\{s=\log x_{1}\right\}$ and $\left\{s=2 \log x-\log x_{1}\right\}$. We will construct

$$
V^{+}(s, x, y) \sim \sum_{j=1}^{\infty} v_{j}^{+}(x, y)\left(e^{s}-x_{1}\right)_{+}^{j} \quad \text { and } \quad V^{-}(s, x, y) \sim \sum_{j=1}^{\infty} v_{j}^{-}(x, y)\left(x^{2}-x_{1} e^{s}\right)_{+}^{j},
$$

that is $V^{+}$and $V^{-}$are the asymptotic sums of those series, such that, $V^{ \pm}$is supported in a neighborhood of $\Sigma^{ \pm}$(this can be arranged, as in the proof of Borel's lemma), and in the set

$$
\begin{gathered}
D_{\epsilon}=\left\{\log x \leq s<\log x_{1}+\log 4,0 \leq x \leq \epsilon / 2\right\} \\
P V^{ \pm} \in C^{\infty}\left(D_{\epsilon}\right), \quad v(s, x, y)-V^{+}(s, x, y)-V^{-}(s, x, y) \in C^{\infty}\left(D_{\epsilon}\right),
\end{gathered}
$$

where $P$ is defined in (4.5). Moreover they satisfy

$$
\begin{gathered}
V^{+}(\log x, x, y)+V^{-}(\log x, x, y) \in C_{0}^{\infty}, \\
\frac{\partial V^{+}}{\partial s}(\log x, x, y)+\frac{\partial V^{-}}{\partial s}(\log x, x, y)-x^{-\frac{n}{2}} w(x, y)\left(x-x_{1}\right)_{+}^{0} \in C_{0}^{\infty} .
\end{gathered}
$$

Once this is accomplished, we then have

$$
\begin{gathered}
P\left(v-V^{+}-V^{-}\right) \in C^{\infty}\left(D_{\epsilon}\right), \\
\left.\left(v-V^{+}-V^{-}\right)\right|_{s=\log x} \in C_{0}^{\infty}(\stackrel{\circ}{X}),\left.\quad \frac{\partial\left(v-V^{+}-V^{-}\right)}{\partial s}\right|_{s=\log x} \in C_{0}^{\infty}(\stackrel{\circ}{X}) .
\end{gathered}
$$

Then the energy estimates from section 4 adapted to the case where the right hand side is not equal to zero, but is smooth up to $M$, gives that $\left.\left(v-V^{+}-V^{-}\right)\right|_{x=0}$ is $C^{\infty}$ in $\left(-\infty, \log x_{1}+\log 4\right) \times M$.

We have

$$
\begin{aligned}
V^{+}(\log x, x, y)+V^{-}(\log x, x, y) & \sim \sum_{j=1}^{\infty}\left(v_{j}^{+}(x, y)+x^{j} v_{j}^{-}(x, y)\right)\left(x-x_{1}\right)_{+}^{j} \sim \sum_{j=1}^{\infty} Z_{j}\left(x_{1}, y\right)\left(x-x_{1}\right)_{+}^{j}, \\
\text { with } Z_{j}\left(x_{1}, y\right) & =\sum_{k+m=j, k \geq 0, m \geq 1} \frac{1}{k !} \frac{\partial^{k}}{\partial x^{k}}\left(v_{m}^{+}+x^{m} v_{m}^{-}\right)\left(x_{1}, y\right)
\end{aligned}
$$

and

$$
\frac{\partial V^{+}}{\partial s}(s, x, y)+\frac{\partial V^{-}}{\partial s}(s, x, y) \sim \sum_{j=1}^{\infty} j e^{s} v_{j}^{+}(x, y)\left(e^{s}-x_{1}\right)_{+}^{j-1}-\sum_{j=1}^{\infty} j x_{1} e^{s} v_{j}^{-}(x, y)\left(x^{2}-x_{1} e^{s}\right)_{+}^{j-1}
$$


So, in particular, when $s=\log x$,

$$
\begin{gathered}
\frac{\partial V^{+}}{\partial s}(\log x, x, y)+\frac{\partial V^{-}}{\partial s}(\log x, x, y) \sim \sum_{j=1}^{\infty}\left(j x v_{j}^{+}(x, y)-j x_{1} x^{j} v_{j}^{-}(x, y)\right)\left(x-x_{1}\right)_{+}^{j-1} \sim \\
\quad \sum_{j=1}^{\infty} M_{j}\left(x_{1}, y\right)\left(x-x_{1}\right)_{+}^{j-1}, \quad \text { where } \\
M_{j}\left(x_{1}, y\right)=\sum_{m+k=j, m \geq 1, k \geq 0} \frac{m}{k !} \frac{\partial^{k}}{\partial x^{k}}\left(x v_{m}^{+}-x_{1} x^{m} v_{m}^{-}\right)\left(x_{1}, y\right) .
\end{gathered}
$$

Since

$$
x^{-\frac{n}{2}} w(x, y)\left(x-x_{1}\right)_{+}^{0} \sim \sum_{j=1}^{\infty} \frac{1}{(j-1) !} \frac{\partial^{j-1}}{\partial x^{j-1}}\left(x^{-\frac{n}{2}} w\right)\left(x_{1}, y\right)\left(x-x_{1}\right)_{+}^{j-1},
$$

we conclude from (8.16), (8.17) and (8.18) that conditions (8.15) translate into

$$
\begin{gathered}
Z_{j}\left(x_{1}, y\right)=\sum_{k+m=j, k \geq 0, m \geq 1} \frac{1}{k !} \frac{\partial^{k}}{\partial x^{k}}\left(v_{m}^{+}+x^{m} v_{m}^{-}\right)\left(x_{1}, y\right)=0, \quad j \in \mathbb{N}, \\
M_{j}\left(x_{1}, y\right)=\sum_{m+k=j, m \geq 1, k \geq 0} \frac{m}{k !} \frac{\partial^{k}}{\partial x^{k}}\left(x v_{m}^{+}-x_{1} x^{m} v_{m}^{-}\right)\left(x_{1}, y\right)=\frac{1}{(j-1) !} \frac{\partial^{j-1}}{\partial x^{j-1}}\left(x^{-\frac{n}{2}} w\right)\left(x_{1}, y\right), \quad j \in \mathbb{N} .
\end{gathered}
$$

In particular, the terms with $j=1$ satisfy

$$
v_{1}^{+}\left(x_{1}, y\right)+x_{1} v_{1}^{-}\left(x_{1}, y\right)=0, \quad \text { and } \quad x_{1} v_{1}^{+}\left(x_{1}, y\right)-x_{1}^{2} v_{1}^{-}\left(x_{1}, y\right)=x_{1}^{-\frac{n}{2}} w\left(x_{1}, y\right) .
$$

Hence

$$
v_{1}^{+}\left(x_{1}, y\right)=-x_{1} v_{1}^{-}\left(x_{1}, y\right)=\frac{1}{2} x_{1}^{-\frac{n}{2}-1} w\left(x_{1}, y\right)
$$

Let

$$
Q=P-2 \frac{\partial}{\partial x} \frac{\partial}{\partial s}-A \frac{\partial}{\partial s}
$$

That is, $Q$ is the part of $P$ that does not have derivatives in $s$. As discussed above, the singularity of $v$ in $\Sigma^{-}$will not hit the boundary for $s \in\left(\log x_{1}, \log x_{1}+\log 4\right)$. Since we are interested on the singularity of $\mathcal{R}_{+}\left(0, \chi_{x_{1}} w\right)$ at $s=\log x_{1}$, we will restrict our computations to $V^{+}$, but keeping in mind that, at least for $x<\epsilon$ and $s<\log x_{1}+\log 4$, similar computations also hold for $V^{-}$.

Since $A$ and the coefficients of $Q$ do not depend on $s$,

$$
\begin{gathered}
P V^{+}(s, x, y) \sim x_{1}\left(2 \frac{\partial}{\partial x} v_{1}^{+}+A v_{1}^{+}\right)\left(e^{s}-x_{1}\right)_{+}^{0}+ \\
\sum_{j=1}^{\infty}\left((j+1) x_{1}\left(2 \frac{\partial}{\partial x}+A\right) v_{j+1}^{+}+\left(2 j \frac{\partial}{\partial x}+j A+Q\right) v_{j}^{+}\right)\left(e^{s}-x_{1}\right)_{+}^{j} \sim 0 \quad \text { in } \quad x<x_{1} .
\end{gathered}
$$

Since we want $P V^{+}(s, x, y) \in C^{\infty}$ in $\left\{x<x_{1}\right\}$, all the coefficients in (8.21) must be equal to zero, and so we get the transport equations for $v_{j}^{+}, j=1,2, \ldots$, which are

$$
\begin{gathered}
x_{1}\left(2 \frac{\partial}{\partial x}+A\right) v_{1}^{+}(x, y)=0, \quad x<x_{1}, \\
\text { and } \\
(j+1) x_{1}\left(2 \frac{\partial}{\partial x}+A\right) v_{j+1}^{+}+\left(2 j \frac{\partial}{\partial x}+j A+Q\right) v_{j}^{+}=0, \quad x<x_{1},
\end{gathered}
$$


with the initial conditions $v_{j}^{+}\left(x_{1}, y\right), j=1,2, \ldots$, given by [8.19).

Since $A=\frac{1}{2} \frac{1}{|h|} \frac{\partial|h|}{\partial x}$, we deduce from (8.22) and (8.20) that

$$
v_{1}^{+}(x, y)=\frac{1}{2} \frac{|h|^{\frac{1}{4}}\left(x_{1}, y\right)}{|h|^{\frac{1}{4}}(x, y)} x_{1}^{-\frac{n}{2}-1} w\left(x_{1}, y\right), \quad x \leq x_{1} .
$$

But

$$
\begin{gathered}
\frac{\partial}{\partial s} V^{+}(s, x, y) \sim \sum_{j=1}^{\infty} j e^{s} v_{j}^{+}(x, y)\left(e^{s}-x_{1}\right)_{+}^{j-1}=\sum_{j=1}^{\infty} j\left(e^{s}-x_{1}+x_{1}\right) v_{j}^{+}(x, y)\left(e^{s}-x_{1}\right)_{+}^{j-1} \sim \\
x_{1} v_{1}^{+}(x, y)\left(e^{s}-x_{1}\right)_{+}^{0}+\sum_{j=1}^{\infty}\left((j+1) x_{1} v_{j+1}^{+}(x, y)+j v_{j}^{+}(x, y)\right)\left(e^{s}-x_{1}\right)_{+}^{j} .
\end{gathered}
$$

The highest singularity of $\mathcal{R}_{+}\left(0, \chi_{x_{1}} w\right)=\left.\frac{\partial v}{\partial s}\right|_{\partial X}$ at $s=\log x_{1}$ is the highest singularity of $\frac{\partial}{\partial s} V^{+}(s, 0, y)$, which is

$$
x_{1} v_{1}^{+}(0, y)\left(e^{s}-x_{1}\right)_{+}^{0}=\frac{1}{2} \frac{|h|^{\frac{1}{4}}\left(x_{1}, y\right)}{|h|^{\frac{1}{4}}(0, y)} x_{1}^{-\frac{n}{2}} w\left(x_{1}, y\right)\left(e^{s}-x_{1}\right)_{+}^{0} .
$$

This gives (8.14) and the Lemma is proved.

Now we can finish the proof of Proposition 8.6

Proof. We know from Lemma 8.9 that there exists $\epsilon>0$ such that for any $\phi \in C_{0}^{\infty}(\mathbb{R})$ even, and $F \in \mathcal{M}^{b}$, then

$$
\frac{\left|h_{1}\right|^{\frac{1}{4}}(x, y)}{\left|h_{1}\right|^{\frac{1}{4}}(0, y)} \psi_{1}\left(\Delta_{g_{1}}-\frac{n^{2}}{4}\right) \mathcal{R}_{1,-}^{-1} F(x, y)=\frac{\left|h_{2}\right|^{\frac{1}{4}}(x, y)}{\left|h_{2}\right|^{\frac{1}{4}}(0, y)} \psi_{1}\left(\Delta_{g_{2}}-\frac{n^{2}}{4}\right) \mathcal{R}_{2,-}^{-1} F(x, y), \quad(x, y) \quad(0, \epsilon) \times \partial X,
$$

where $\psi_{1}$ is determined by (7.2). Now take a sequence $\phi_{m}$ such that $\phi_{m} * F \rightarrow F$ in $L^{2}(\mathbb{R} \times \partial X)$ as $m \rightarrow \infty$. Then, by the continuity of $\mathcal{R}_{j,-}, j=1,2$,

$$
\psi_{1, m}\left(\Delta_{g_{j}}-\frac{n^{2}}{4}\right) \mathcal{R}_{j,-}^{-1} F \rightarrow \mathcal{R}_{j,-}^{-1} F \text { in } L^{2}\left(X_{j}\right) \text { with respect to } g_{j} .
$$

Since $\left|h_{1}\right|(0, y)=\left|h_{2}\right|(0, y)$, 8.10) holds for the backward radiation field.

Let $F=\mathcal{S}^{-1} G=\mathcal{R}_{1,-} \mathcal{R}_{1,+}^{-1} G=\mathcal{R}_{2,-} \mathcal{R}_{2,+}^{-1} G$, then

$$
\left|h_{1}\right|^{\frac{1}{4}}(x, y) \mathcal{R}_{1,+}^{-1} G=\left|h_{2}\right|^{\frac{1}{4}}(x, y) \mathcal{R}_{2,+}^{-1} G, \quad \text { in } \quad(0, \epsilon) \times \partial X .
$$

Since $\mathcal{S}$ is unitary and $G$ is arbitrary, therefore (8.10) holds for the forward radiation field. This ends the proof of Proposition 8.6

Since $\left|h_{1}\right|=\left|h_{2}\right|$ the following is an immediate consequence of Proposition 8.6

Proposition 8.10. Let $\left(X_{1}, g_{1}\right)$ and $\left(X_{2}, g_{2}\right)$ satisfy the hypotheses of Theorem 8.1 be such that $\Delta_{g_{j}}$, $j=1,2$, have no eigenvalues. Then there exists $\epsilon>0$ such that in coordinates for which 8.4 holds, the Schwartz kernels of the radiation fields $\mathcal{R}_{j, \pm}(s, y, z)$, for $(s, y) \in \mathbb{R} \times M, z \in(0, \epsilon) \times M$, satisfy

$$
\mathcal{R}_{1,+}(s, y, z)=\mathcal{R}_{2,+}(s, y, z) \text { and } \mathcal{R}_{1,-}(s, y, z)=\mathcal{R}_{2,-}(s, y, z) .
$$

Next we want to show that $\Psi$ extends to a global diffeomorphism from $X_{1}$ to $X_{2}$, as claimed in Theorem 8.1 We could follow the method of [4 5, 29, see also [30, 31, and construct the diffeomorphism. However it is easier to show that one can apply their result to construct $\Psi$. 


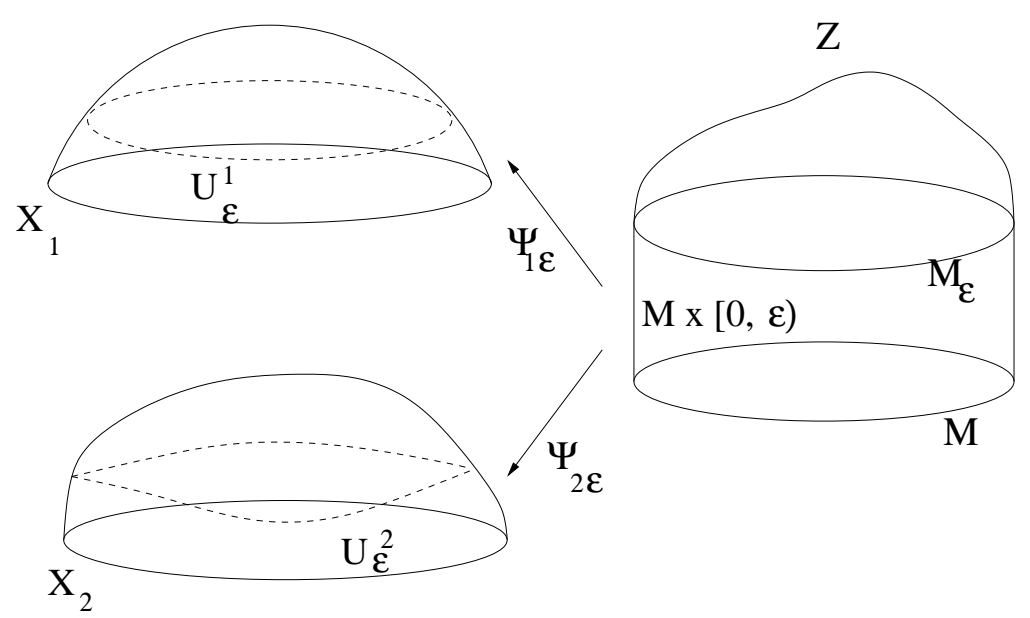

Figure 6 . The maps $\Psi_{j, \epsilon}, j=1,2$ and their extension to $Z$.

Proposition 8.11. (44, 5, 29]) Let $(Z, g)$ be a smooth compact Riemannian manifold with boundary with boundary $\partial Z$. Let $\nu_{j}, \nu_{j} \leq \nu_{j+1}, j \in \mathbb{N}$, denote the Neumann eigenvalues of the operator $\Delta_{g}$, on $Z$ and let $\gamma_{j}$ denote the corresponding eigenfunctions. Then $(Z, g)$ is uniquely determined, modulo a diffeomorphism that is the identity at $\partial Z$, by the collection

$$
\nu_{j} \text { and }\left.\gamma_{j}\right|_{\partial Z}, j \in \mathbb{N} \backslash J \text {, where } J \text { is a finite subset. }
$$

This result is proved in [4, 5] for $J=\emptyset$ and in [29, 30] for $J \neq \emptyset$.

So far we have shown that there exists a diffeomorphism $\Psi$ of a neighborhood of $\partial X$ such that

$$
\Psi^{*}\left(\left.g_{1}\right|_{U_{1, \epsilon}}\right)=\left.g_{2}\right|_{U_{2, \epsilon}} \text { near } \quad M
$$

Observe that $X_{j, \epsilon}=X_{j} \backslash U_{j, \epsilon}$ are smooth compact manifolds with boundary and their boundaries $\partial X_{1, \epsilon}$, $\partial X_{2, \epsilon}$ can be identified by the diffeomorphisms with $M \times\{\epsilon\}=M_{\epsilon}$, see figure 6

We think of $M_{\epsilon}$ as the boundary of some smooth compact manifold $Z$. We will prove that the fact that the two metrics have the same scattering matrix imply that $\Delta_{g_{i}}$ in $X_{i, \epsilon}, i=1,2$, have the same Neumann spectral data in $M_{\epsilon}$, that is, they have the same eigenvalues and the same traces of the eigenfunctions. Then Proposition 8.11 with $J=\emptyset$, put together with Proposition 8.2 proves Theorem 8.1 We should also remark that method of proof of Proposition 8.11 guarantees that the resulting map is $C^{\infty}$.

As in 42], we recall that the graph of the Calderón projector of $\Delta_{g_{j}}-\lambda^{2}-\frac{n^{2}}{4}$ in $X_{j, \epsilon}, j=1,2$, denoted by $C_{j, \lambda}$, is the closed subspace of $L^{2}\left(M_{\epsilon}\right) \times H^{1}\left(M_{\epsilon}\right)$ consisting of $(f, g) \in L^{2}\left(M_{\epsilon}\right) \times H^{1}\left(M_{\epsilon}\right)$ such that there exists $u$ satisfying

$$
\begin{gathered}
\left(\Delta_{g_{j}}-\lambda^{2}-\frac{n^{2}}{4}\right) u=0 \quad \text { in } \quad X_{j, \epsilon}, j=1,2, \\
\left.u\right|_{M_{\epsilon}}=f,\left.\quad \partial_{\nu} u\right|_{M_{\epsilon}}=g .
\end{gathered}
$$

Here $\partial_{\nu} u$ denotes the normal derivative of $u$ at $M_{\epsilon}$. We will show that if $A_{1}(\lambda)=A_{2}(\lambda), \lambda \in \mathbb{R} \backslash 0$, then $C_{1, \lambda}=C_{2, \lambda}, \lambda \in \mathbb{R} \backslash 0$. But the Calderón projector depends continuously on $\lambda$ and so does its graph. Thus $C_{1, \lambda}=C_{2, \lambda}, \lambda \in \mathbb{R}$.

Since $\Delta_{g_{j}}, j=1,2$, has no point spectrum in $L^{2}\left(X_{j}\right)$, the operators $\Delta_{g_{j}}-\frac{n^{2}}{4}, j=1,2$, are positive in $X_{j}$. In particular their restriction to $X_{j, \epsilon}$ are also positive. Therefore $\lambda^{2}+\frac{n^{2}}{4}$ is in the Neumann spectrum of $\Delta_{g_{j}}$ in $X_{j, \epsilon}$, if and only if $C_{j, \lambda}$ contains a subspace of pairs of the form $(g, 0), g \neq 0$. Therefore, once 
we prove that $C_{1, \lambda}=C_{2, \lambda}, \lambda \in \mathbb{R}$, then the eigenvalues of the Neumann problem for $\Delta_{g_{1}}$ in $X_{1, \epsilon}$, and the traces of the corresponding eigenfunctions on $M_{\epsilon}$, are equal to the corresponding ones of $\Delta_{g_{2}}$ in $X_{2, \epsilon}$. Then Proposition 8.11 with $J=\emptyset$, can be used to prove Theorem 8.1

To prove that $C_{1, \lambda}=C_{2, \lambda}, \lambda \in \mathbb{R} \backslash 0$, we apply the same argument used in the proof of Lemma 3.2, chapter 3.8 of [44, see also the proof of Lemma 2.1 of [52], to show that for any $\lambda \neq 0$, the set of functions given by

$$
v_{j}(z, \lambda)=\int_{M} E_{j}^{*}\left(\frac{n}{2}+i \lambda\right)(z, y) \phi(y), \quad j=1,2, \quad \phi \in C^{\infty}(M),
$$

where $E_{j}^{*}$ is the Eisenstein Function, or Poisson operator, which is the adjoint of the operator $E_{j}$ defined in (6.10), is dense in the set of solutions of

$$
\left(\Delta-\lambda^{2}-\frac{n^{2}}{4}\right) u=0 \quad \text { in } \quad X_{j, \epsilon}, \quad j=1,2,
$$

in the Sobolev space $H^{k}\left(X_{j, \epsilon}\right)$, for any $k \geq 2$.

The dual to $H^{k}\left(X_{j, \epsilon}\right)$ can be identified with the space of $f \in H^{-k}\left(X_{j}\right)$ supported in $X_{j, \epsilon}$. If such $f$ satisfies $\langle f, v\rangle=0$ for all $v$ given by (8.29), then $E_{j}\left(\frac{n}{2}+i \lambda\right) f=0$. This implies that the function $h=R\left(\frac{n}{2}+i \lambda\right) f$ vanishes to infinite order at $\partial X$. Notice that $f$ is supported in $X_{j, \epsilon}$, so $h$ is smooth near $\partial X$. Then unique continuation, see Theorem 14 of [40], implies that $h$ is supported in $X_{j, \epsilon}$ and therefore $f=\left(\Delta_{g_{j}}-\lambda^{2}-\frac{n^{2}}{4}\right) h$, with $h$ supported in $X_{j, \epsilon}$. Then $\langle f, u\rangle=0$ for any $u$ satisfying (8.30).

Since $E_{j}^{*}\left(\frac{n}{2}+i \lambda\right)(z, y)$ is the partial Fourier transform in $s$ of $\mathcal{R}_{j,-}^{-1}(s, y, z), j=1,2$, Proposition 8.10 implies that for all $v_{j}, j=1,2$, given by (8.29), which we know is smooth in $X_{j}$, satisfy $v_{1}(z, \lambda)=v_{2}(z, \lambda)$, $z=(x, y) \in(0, \epsilon) \times M, \lambda \in \mathbb{R} \backslash 0$. Therefore their traces and normal derivatives at $M_{\epsilon}$ are equal, and the density of this set implies that the same is true for solutions of (8.30). Thus $C_{1, \lambda}=C_{2, \lambda}, \lambda \in \mathbb{R} \backslash 0$ and this proves our claim.

8.3. The general case. Now we remove the extra assumption on the non-existence of eigenvalues. Let $(X, g)$ be an asymptotically hyperbolic manifold. The only poles of the resolvent $R\left(\frac{n}{2}+i \lambda\right)=$ $\left(\Delta_{g}-\frac{n^{2}}{4}-\lambda^{2}\right)^{-1}$ in $\{\Im \lambda<0\}$ correspond to the finitely many eigenvalues of $\Delta_{g}$. Proposition 3.6 of [16] states that if $\lambda_{0}>0$ is such that $\frac{n^{2}}{4}-\lambda_{0}^{2}$ is an eigenvalue of $\Delta_{g}$ then the scattering matrix has a pole at $-i \lambda_{0}$ and its residue is given by

$$
\operatorname{Res}_{-i \lambda_{0}} A(\lambda)=\left\{\begin{array}{rll}
\Pi_{\lambda_{0}}, & \text { if } & \lambda_{0} \notin \mathbb{N} / 2, \\
\Pi_{\lambda_{0}}-p_{l}, & \text { if } & \lambda_{0}=\frac{l}{2}, l \in \mathbb{N},
\end{array}\right.
$$

where $p_{l}$ is a differential operator whose coefficients depend on the tensor $h$, defined in (8.4), and its derivatives at $\partial X$, and the Schwartz kernel of $\Pi_{\lambda_{0}}$ is

$$
K\left(\Pi_{\lambda_{0}}\right)\left(y, y^{\prime}\right)=2 \lambda_{0} \sum_{j=1}^{N} \phi_{j}^{0} \otimes \phi_{j}^{0}\left(y, y^{\prime}\right), \quad \phi_{j}^{0}(y)=\left.x^{-\frac{n}{2}-\lambda_{0}} \phi_{j}(x, y)\right|_{x=0} .
$$

Here $N$ is the multiplicity of the eigenvalue $\frac{n^{2}}{4}-\lambda_{0}^{2}$ and $\phi_{j}, 1 \leq j \leq N$, are the corresponding orthonormalized eigenfunctions.

If two asymptotically hyperbolic manifolds $\left(X_{1}, g_{1}\right)$ and $\left(X_{2}, g_{2}\right)$ have the same scattering matrix $A_{1}(\lambda)=A_{2}(\lambda)$ for all $\lambda \in \mathbb{R} \backslash 0$, we know from [28] that in coordinates where (8.4) is satisfied, all derivatives of $h_{1}$ and $h_{2}$ agree at $x=0$. Therefore the operators $p_{l}$ in (8.31) are the same. Thus, (8.31) and (8.32), and the meromorphic continuation of the scattering matrix, show that $\Delta_{g_{1}}$ and $\Delta_{g_{2}}$ have the same eigenvalues, with the same multiplicity. Moreover, (8.32) implies that if $\phi_{j}$, and $\psi_{j}$, 
$1 \leq j \leq N$, are orthonormal sets of eigenfunctions of $\Delta_{g_{1}}$ and $\Delta_{g_{2}}$, respectively, corresponding to the eigenvalue $\frac{n^{2}}{4}-\lambda_{0}^{2}$, then there exists a constant orthogonal $N \times N$ matrix $A$ such that $\Phi^{0}=A \Psi^{0}$, where $\left(\Phi^{0}\right)^{T}=\left(\phi_{1}^{0}, \phi_{2}^{0}, \ldots, \phi_{N}^{0}\right)$, and $\left(\Psi^{0}\right)^{T}=\left(\psi_{1}^{0}, \psi_{2}^{0}, \ldots, \psi_{N}^{0}\right)$. So by redefining one set of eigenfunctions from let us say, $\Psi$ to $A \Psi$, where $\Psi^{T}=\left(\psi_{1}, \psi_{2}, \ldots, \psi_{N}\right)$, we may assume that

$$
\phi_{j}^{0}(y)=\psi_{j}^{0}(y), \quad j=1,2, \ldots, N .
$$

We remark that this does not change the orthonormality of the eigenfunctions in $X_{2}$ because $A$ is orthogonal. Let us denote

$$
\begin{aligned}
& \mu_{j}=\frac{n^{2}}{4}-\lambda_{j}^{2}, \quad \phi_{j}, \quad 1 \leq j \leq L, \quad \text { all the eigenvalues and eigenfunctions of } \Delta_{g_{1}} \\
& \mu_{j}=\frac{n^{2}}{4}-\lambda_{j}^{2}, \quad \psi_{j}, \quad 1 \leq j \leq L, \quad \text { all the eigenvalues and eigenfunctions of } \Delta_{g_{2}}
\end{aligned}
$$

with $\mu_{1} \leq \mu_{2} \leq \ldots \leq \mu_{L}$, and the eigenfunctions $\phi_{j}$ and $\psi_{j}$ chosen to satisfy [8.33).

When there are eigenfunctions, Lemma 8.7 is no longer valid. To present the correct statement we begin with the following lemma, which was suggested and proved by one of the referees,

Lemma 8.12. Let $V$ be a finite dimensional subspace of a Hilbert space $H$ and let $Q$ be the orthogonal projector onto $V$. Let $P_{t}, t \in[0, a]$, be a strongly continuous family of projections with $P_{0}=\operatorname{Id}$ and $P_{t} P_{t^{\prime}}=P_{t}$ if $t \leq t^{\prime}$. Then there exists $\epsilon \in(0, a)$, and a unique continuous family of bounded operators $T(t): H \longrightarrow V, t \in[0, \epsilon]$, such that $Q P_{t}(\mathrm{Id}-T(t))=0$. Moreover, if $f_{m} \in H$ is a bounded sequence, then there exists a subsequence $f_{m^{\prime}}$ such that $T(t) f_{m^{\prime}}$ converges uniformly in $[0, \epsilon]$.

Proof. As $\left.Q\left(P_{t}\right)\right|_{V}$ is continuous and $\left.Q\left(P_{0}\right)\right|_{V}=\left.\operatorname{Id}\right|_{V}$, there exists $\epsilon \in(0, a)$ such that $Q P_{t}: V \longrightarrow V$ is invertible on $V$ for $t \in[0, \epsilon]$. If $R(t)$ is its inverse, then $R(t)$ is continuous for $t \in[0, \epsilon]$, and

$$
Q P_{t}\left(\mathrm{Id}-R(t) Q P_{t}\right)=Q P_{t}-\left(Q P_{t}\right) R(t) Q P_{t}=0 .
$$

So we take $T(t)=R(t) Q P_{t}$. Then $T(t)$ is bounded and continuous in $t$. If $\widetilde{T}(t)$ is another solution, $Q P_{t}(T(t)-\widetilde{T}(t))=0$, so multiplying by $R(t)$ on the left, we get $T(t)-\widetilde{T}(t)=0$.

If $f_{m}$ is a bounded sequence of functions on $H$, then $P_{t} f_{m}$ is bounded. From the strong continuity of $P_{t}, P_{t} f_{m}$ is equicontinuous and hence it has a convergent subsequence $P_{t} f_{m^{\prime}}$. Thus $T(t) f_{m^{\prime}}=R(t) Q P_{t} f_{m^{\prime}}$ converges. This ends the proof of the lemma.

The application of this lemma that we have in mind is:

Corollary 8.13. Let $(X, g)$ be an asymptotically hyperbolic manifold and let $x$ be a defining function of $\partial X$ for which (2.4) holds. Let $V=L_{\mathrm{pp}}^{2}(X)$, be the space spanned by the set of orthonormal eigenfunctions of $\Delta_{g}, \zeta_{j} \in L^{2}(X), 1 \leq j \leq N$. Let $\chi_{t}$ denote the characteristic function of the set $\{x \geq t\}$ and let $P_{t} f=$ $\chi_{t} f$. Then there exists $\epsilon>0$, and a unique continuous family of bounded operators $T(t): L^{2}(X) \longrightarrow V$, $t \in[0, \epsilon]$, such that

$$
\left\langle\chi_{t}(f-T(t) f), \zeta_{k}\right\rangle=0, \quad k=1,2, \ldots, N .
$$

Moreover, any bounded sequence $f_{m} \in L^{2}(X), m=1,2, \ldots$, has a subsequence $f_{m^{\prime}}$ such that $T(t)\left(f_{m^{\prime}}\right)$ converges uniformly in $[0, \epsilon], j=1,2, \ldots, N$. In this case the operators $R(t)$ and $Q P_{t}$ are given by

$$
R(t)=\left(R(t)_{i j}\right), \quad R(t)_{i j}=\left\langle\chi_{t} \zeta_{i}, \zeta_{j}\right\rangle, \quad\left(Q P_{t} f\right)^{T}=\left(\left\langle\chi_{t} f, \zeta_{1}\right\rangle,\left\langle\chi_{t} f, \zeta_{2}\right\rangle, \ldots,\left\langle\chi_{t} f, \zeta_{N}\right\rangle\right) .
$$

When there exist eigenfunctions, Lemma 8.7 has to be replaced by 
Lemma 8.14. Let $(X, g)$ be an asymptotically hyperbolic manifold and let $\left\{\zeta_{j}: 1 \leq j \leq N\right\}$ denote the $L^{2}(X)$ eigenfunctions of $\Delta_{g}$. Let $\mathcal{P}_{x_{1}}^{b}$ denote the orthogonal projector

$$
\mathcal{P}_{x_{1}}^{b}: \mathcal{M}^{b} \longrightarrow \mathcal{M}^{b}\left(x_{1}\right) .
$$

Let $\chi_{t}$ be the characteristic function of the set $\{x \geq t\}$. Then there exists $\epsilon>0$ such that for every $x_{1} \in(0, \epsilon)$ and every $f \in L_{\mathrm{ac}}^{2}(X)$

$$
\begin{aligned}
& \mathcal{P}_{x_{1}}^{b} \mathcal{R}_{-}(0, f)=\mathcal{R}_{-}\left(0, f_{x_{1}}\right), \quad \text { where } \\
& f_{x_{1}}(x, y)=\chi_{x_{1}}(x, y)\left(\operatorname{Id}-T\left(x_{1}\right)\right) f,
\end{aligned}
$$

and $T\left(x_{1}\right)$ is the family of operators given by Corollary 8.13 .

Proof. We follow the proof of Lemma 8.7 We know from Lemma 8.5 that there exists $f_{x_{1}} \in L_{\mathrm{ac}}^{2}(X)$ supported in $\left\{x \geq x_{1}\right\}$ such that $\mathcal{P}_{x_{1}}^{b} \mathcal{R}_{-}(0, f)=\mathcal{R}_{-}\left(0, f_{x_{1}}\right)$ and that

$$
\left\langle f_{x_{1}}-f, g\right\rangle=0 \text { for all } g \in C_{0}^{\infty}(\stackrel{\circ}{X}) \cap L_{\text {ac }}^{2}(X) \text { supported in }\left\{x_{1} \leq x\right\} .
$$

Therefore there exist constants $\alpha_{j}\left(x_{1}\right), j=1,2, \ldots, L$, such that

$$
f_{x_{1}}=\chi_{x_{1}}\left(f+\sum_{j=1}^{N} \alpha_{j}\left(x_{1}\right) \zeta_{j}\right) .
$$

Since $f_{x_{1}} \in L_{\mathrm{ac}}^{2}\left(X_{1}\right)$, it follows that $\left\langle f_{x_{1}}, \zeta_{k}\right\rangle=0,1 \leq k \leq N$. Then, by uniqueness, $\sum_{j=1}^{N} \alpha_{j}\left(x_{1}\right) \zeta_{j}=$ $-T\left(x_{1}\right)(f)$, is the family of operators given by Corollary 8.13

If there are eigenvalues, then Lemma 8.9 is replaced by

Lemma 8.15. Let $(X, g)$ be an asymptotically hyperbolic manifold and let $x$ be a defining function of $\partial X$ such that (2.4) holds. Let $\mathcal{R}_{ \pm}$be the radiation fields with respect to $x$. Then there exists $\epsilon>0$ such that for all $F \in \mathcal{M}^{b}, \phi \in C_{0}^{\infty}(\mathbb{R})$ even, and $\psi_{1}$ defined by $(\mathbf{7 . 2})$, and any $x_{1} \in(0, \epsilon / 4)$,

$$
\begin{gathered}
\mathcal{R}_{+} \mathcal{R}_{-}^{-1}\left(\mathcal{P}_{x_{1}}^{b} G\right)(s, y)= \\
\frac{1}{2} x_{1}^{-\frac{n}{2}}\left(\operatorname{Id}-T\left(x_{1}\right)\right) w\left(x_{1}, y\right) \frac{\left|h_{1}\right|^{\frac{1}{4}}\left(x_{1}, y\right)}{\left|h_{1}\right|^{\frac{1}{4}}(0, y)}\left(s-\log x_{1}\right)_{+}^{0}+\text { smoother terms, } \\
\text { if } s<\log x_{1}+\log 4, \text { where } G=\phi * F, \quad \text { and } w_{1}=\mathcal{R}_{-}^{-1} G=\psi_{1}\left(\Delta_{g}-\frac{n^{2}}{4}\right) \mathcal{R}_{-}^{-1} F .
\end{gathered}
$$

Here $T\left(x_{1}\right)$ is the operator given by Corollary 8.13. Notice that by (7.3), $G=\phi * F \in \mathcal{M}^{b}$, and that the left hand side of (8.38) is determined by the scattering matrix $A(\lambda)$.

Proof. We follow step by step the proof of Lemma 8.9 We know that the eigenfunctions are smooth in the interior of the manifold. So the only thing that changes is the singularity of the initial data in equation (8.19). The initial data of $v_{1}^{+}$, which was given by (8.20), must, according to (8.37), be replaced by

$$
v^{+}\left(x_{1}, y\right)=\frac{1}{2} x_{1}^{-\frac{n}{2}-1}\left(\mathrm{Id}-T\left(x_{1}\right)\right) w_{1}\left(x_{1}, y\right)
$$

As in the proof of Proposition 8.6 we take a sequence $\phi_{m}$ such that $\phi_{m} * F \rightarrow F$ in $L^{2}(\mathbb{R} \times M)$, in Lemma 8.15, and we obtain 
Proposition 8.16. Let $\left(X_{j}, g_{j}\right), j=1,2$, be asymptotically hyperbolic manifolds satisfying the hypotheses of Theorem 8.1. Let $\mathcal{R}_{j,-}, j=1,2$, denote the corresponding backward radiation fields defined in coordinates in which (8.4) holds. Let $T_{j}(t), j=1,2$, be the operators given by Corollary 8.13. Then there exists $\epsilon>0$ such that for any $F \in \mathcal{M}^{b}$,

$$
\left|h_{1}\right|^{\frac{1}{4}}(x, y)\left(\operatorname{Id}-T_{1}(x)\right) \mathcal{R}_{1,-}^{-1} F(x, y)=\left|h_{2}\right|^{\frac{1}{4}}(x, y)\left(\operatorname{Id}-T_{2}(x)\right) \mathcal{R}_{2,-}^{-1} F(x, y) \quad \text { in }(0, \epsilon) \times M .
$$

We deduce from (8.39) and (8.11) that for every $F \in \mathcal{M}^{b}$,

$$
\begin{gathered}
\left|h_{1}\right|^{\frac{1}{4}}\left(\mathrm{Id}-T_{1}\right) \mathcal{R}_{1,-}^{-1} F(x, y)=\left|h_{2}\right|^{\frac{1}{4}}\left(\mathrm{Id}-T_{2}\right) \mathcal{R}_{2,-}^{-1} F(x, y), \quad(x, y) \in(0, \epsilon) \times M, \quad \text { and } \\
\left|h_{1}\right|^{\frac{1}{4}}\left(\mathrm{Id}-T_{1}\right)\left(\Delta_{g_{1}}-\frac{n^{2}}{4}\right) \mathcal{R}_{1,-}^{-1} F(x, y)=\left|h_{2}\right|^{\frac{1}{4}}\left(\mathrm{Id}-T_{2}\right)\left(\Delta_{g_{2}}-\frac{n^{2}}{4}\right) \mathcal{R}_{2,-}^{-1} F(x, y), \quad(x, y) \in(0, \epsilon) \times M .
\end{gathered}
$$

We will use this to conclude that the tensors $h_{1}$ and $h_{2}$ are equal, and thus prove Proposition 8.2 This is based on the following lemma, which is a direct consequence of Corollary 8.13

Lemma 8.17. For $\delta>0$, let $K_{\delta}$ be the characteristic function of $\{x \leq \delta\}$. Then there exists $\epsilon>0$ such that for every $\delta \in(0, \epsilon)$, the operators $T_{j}, j=1,2$, defined by Corollary 8.13 satisfy

$$
K_{\delta} T_{j}: L^{2}\left(X_{j}\right) \longrightarrow L^{2}([0, \delta] \times M), \quad j=1,2,
$$

where $L^{2}$ is defined with respect to the metric $g_{j}$, and moreover are compact.

We can then prove Proposition 8.2

Proof. We just need to observe that it follows from (8.40) and Lemma 8.17 that for $\epsilon>0$ small,

$$
\begin{gathered}
K_{\epsilon}\left|h_{1}\right|^{\frac{1}{4}} \mathcal{R}_{1,-}^{-1} F(x, y)=K_{\epsilon}\left|h_{2}\right|^{\frac{1}{4}} \mathcal{R}_{2,-}^{-1} F(x, y)+K_{\epsilon} R F(x, y), \quad R \text { compact, and } \\
K_{\epsilon}\left|h_{1}\right|^{\frac{1}{4}}\left(\Delta_{g_{1}}-\frac{n^{2}}{4}\right) \mathcal{R}_{1,-}^{-1} F(x, y)=K_{\epsilon}\left|h_{2}\right|^{\frac{1}{4}}\left(\Delta_{g_{2}}-\frac{n^{2}}{4}\right) \mathcal{R}_{2,-}^{-1} F(x, y)+K_{\epsilon} S F(x, y), \quad S \text { compact. }
\end{gathered}
$$

Let $\delta \in(0, \epsilon)$ and set $\mathcal{R}_{1,-}^{-1} F=f$. We find that for all $f \in L_{\text {ac }}^{2}\left(X_{1}\right)$, with respect to $g_{1}$,

$$
K_{\delta}\left|h_{2}\right|^{\frac{1}{4}}\left(\Delta_{g_{2}}-\frac{n^{2}}{4}\right) \frac{\left|h_{1}\right|^{\frac{1}{4}}}{\left|h_{2}\right|^{\frac{1}{4}}} f=K_{\delta}\left|h_{1}\right|^{\frac{1}{4}}\left(\Delta_{g_{1}}-\frac{n^{2}}{4}\right) f+K_{\delta} \widetilde{S} f, \quad \widetilde{S} \quad \text { compact. }
$$

Let $Q$ be the differential operator

$$
Q f=\left(\left|h_{2}\right|^{\frac{1}{4}}\left(\Delta_{g_{2}}-\frac{n^{2}}{4}\right) \frac{\left|h_{1}\right|^{\frac{1}{4}}}{\left|h_{2}\right|^{\frac{1}{4}}}-\left|h_{1}\right|^{\frac{1}{4}}\left(\Delta_{g_{1}}-\frac{n^{2}}{4}\right)\right) f .
$$

Since any $f \in L^{2}\left(X_{1}\right)$, with respect to $g_{1}$, can be written as $f=\mathcal{P}_{\text {ac }} f+\sum_{j=1}^{N}\left\langle f, \phi_{j}\right\rangle \phi_{j}$, it follows from (8.42) that

$$
K_{\delta} Q f=K_{\delta} Q \mathcal{P}_{\mathrm{ac}} f+\sum_{j=1}^{N}\left\langle f, \phi_{j}\right\rangle K_{\delta} Q \phi_{j}=K_{\delta} \widetilde{S} \mathcal{P}_{\mathrm{ac}} f+\sum_{j=1}^{N}\left\langle f, \phi_{j}\right\rangle K_{\delta} Q \phi_{j}, \quad f \in L^{2}\left(X_{1}\right) .
$$

The first term of the sum is compact because $\widetilde{S}$ is, and the second term is of finite rank. Therefore $K_{\delta} Q: L^{2}\left(X_{1}\right) \longrightarrow H^{-2}([0, \delta] \times M)$ is compact. But $Q$ is also a second order differential operator. This implies that $K_{\delta} Q=0$. See for example exercise 6.2 on page 52 of [51]. Therefore the tensors $h_{1}$ and $h_{2}$ are equal in $[0, \delta] \times M$, and this proves Proposition 8.2 
Now we need to show that the diffeomorphism can be extended to the whole manifold. We will use the same method as in the case of no eigenvalues. We have shown that $\Delta_{g_{1}}=\Delta_{g_{2}}$ in coordinates (8.4) in $(0, \epsilon) \times M$. Using (8.33) and the equation satisfied by the eigenfunctions, it can be shown that (8.33) is satisfied to infinite order, that is all derivatives of the "rescaled eigenfunctions" agree at $\{x=0\}$. Therefore unique continuation for this type of operators, see Theorem 14 of [40, shows that there exists $\epsilon>0$, such that the eigenfunctions of $\Delta_{g_{1}}$ and those of $\Delta_{g_{2}}$ are equal in $[0, \epsilon) \times M$. That is

$$
\phi_{j}(x, y)=\psi_{j}(x, y), \quad 1 \leq j \leq L, \quad(x, y) \in[0, \epsilon) \times M .
$$

We deduce from the first equation in (8.40) that the Schwartz kernels $\mathcal{R}_{j,-}^{-1}(s, y, z),(s, y) \in \mathbb{R} \times M$ and $z=\left(x, y^{\prime}\right) \in(0, \epsilon) \times M$ satisfy

$$
\left(I-T_{1}\right) \mathcal{R}_{1,-}^{-1}(s, y, z)=\left(I-T_{2}\right) \mathcal{R}_{2,-}^{-1}(s, y, z) .
$$

Since $T_{j}, j=1,2$, is a linear operator, we may take Fourier transform in $s$ of (8.44) and deduce that the Schwartz kernels $E_{j}^{*}\left(\frac{n}{2}+i \lambda\right)(z, y), j=1,2,(\lambda, y) \in \mathbb{R} \backslash 0 \times M$, and $z=\left(x, y^{\prime}\right) \in(0, \epsilon) \times M$ satisfy

$$
\left(I-T_{1}\right) E_{1}^{*}\left(\frac{n}{2}+i \lambda\right)(z, y)=\left(I-T_{2}\right) E_{2}^{*}\left(\frac{n}{2}+i \lambda\right)(z, y) .
$$

We will use this to prove

Proposition 8.18. There exists $\epsilon>0$ such that for every $\lambda \in \mathbb{R} \backslash 0, y \in M$ and $z=\left(x, y^{\prime}\right) \in(0, \epsilon) \times M$,

$$
E_{1}^{*}\left(\frac{n}{2}+i \lambda\right)(z, y)=E_{2}^{*}\left(\frac{n}{2}+i \lambda\right)(z, y) .
$$

Proof. Let $\phi \in C^{\infty}(M)$ and let $v_{j}(z, \lambda), j=1,2$, be the functions given by [8.29). We will show that there exists $\epsilon>0$ such that for $\delta \in(0, \epsilon)$, there exists $\Lambda=\Lambda(\delta)>0$ such that

$$
v_{1}\left(\delta, y^{\prime}, \lambda\right)=v_{2}\left(\delta, y^{\prime}, \lambda\right), \quad \forall y^{\prime} \in M, \quad \text { and }|\lambda|>\Lambda .
$$

Since by the analytic continuation of $E_{j}\left(\frac{n}{2}+i \lambda\right), \lambda \in \mathbb{R} \backslash 0, v_{j}(z, \lambda), j=1,2$, is real analytic in $\lambda \in \mathbb{R} \backslash 0$, for each $z$, it follows that [8.47] holds for every $\lambda \in \mathbb{R} \backslash 0$. Since $\delta$ is arbitrary, 8.46) follows.

Equation 8.45) implies that

$$
v_{1}(z, \lambda)-v_{2}(z, \lambda)=T_{1} v_{1}(z, \lambda)-T_{2} v_{2}(z, \lambda), \quad z=\left(x, y^{\prime}\right) \in(0, \epsilon) \times M, \quad \lambda \in \mathbb{R} \backslash 0 .
$$

Let us denote

$$
\begin{gathered}
\Phi(x, y)=\left(\phi_{1}(x, y), \ldots, \phi_{L}(x, y)\right), \quad \Psi(x, y)=\left(\psi_{1}(x, y), \ldots, \psi_{L}(x, y)\right), \\
T_{1} v_{1}(z, \lambda)=\sum_{j=1}^{L} C_{j}^{1}(x, \lambda) \phi_{j}(x, y), \quad T_{2} v_{2}(z, \lambda)=\sum_{j=1}^{L} C_{j}^{2}(x, \lambda) \psi_{j}(x, y),
\end{gathered}
$$

where, by equation 8.36

$$
\begin{gathered}
C^{j}(x, \lambda) \stackrel{\text { def }}{=} C^{j}\left(x, v_{j}(z, \lambda)\right)=\left[R^{j}(x)\right]^{-1} F^{j}(x, \lambda), \quad j=1,2, \text { where } \\
F^{1}(x, \lambda)^{T} \stackrel{\text { def }}{=} F^{1}\left(x, v_{1}(z, \lambda)\right)^{T}=\left(\left\langle\chi_{x} v_{1}(z, \lambda), \phi_{1}\right\rangle, \ldots,\left\langle\chi_{x} v_{1}(z, \lambda), \phi_{L}\right\rangle\right), \\
F^{2}(x, \lambda)^{T} \stackrel{\text { def }}{=} F^{2}\left(x, v_{2}(z, \lambda)\right)^{T}=\left(\left\langle\chi_{x} v_{2}(z, \lambda), \psi_{1}\right\rangle, \ldots,\left\langle\chi_{x} v_{2}(z, \lambda), \psi_{L}\right\rangle\right) \\
R^{1}(x)=\left(R_{i j}^{1}(x)\right), \quad R_{i j}^{1}(x)=\left\langle\chi_{x} \phi_{j}, \phi_{j}\right\rangle, \quad R^{2}(x)=\left(R_{i j}^{2}(x)\right), \quad R_{i j}^{2}(x)=\left\langle\chi_{x} \psi_{j}, \psi_{j}\right\rangle,
\end{gathered}
$$

We know that $\left\langle\phi_{i}, \phi_{j}\right\rangle=\left\langle\psi_{i}, \psi_{j}\right\rangle=\delta_{i j}$, we also know that if $x<\epsilon$, then $\left(1-\chi_{x}\right) \phi_{i}=\left(1-\chi_{x}\right) \psi_{i}$. But,

$$
\begin{aligned}
& \left\langle\chi_{x} \phi_{i}, \phi_{j}\right\rangle=\left\langle\phi_{i}, \phi_{j}\right\rangle-\left\langle\left(1-\chi_{x}\right) \phi_{i}, \phi_{j}\right\rangle=\left\langle\phi_{i}, \phi_{j}\right\rangle-\left\langle\left(1-\chi_{x}\right) \phi_{i},\left(1-\chi_{x}\right) \phi_{j}\right\rangle= \\
& \left\langle\psi_{i}, \psi_{j}\right\rangle-\left\langle\left(1-\chi_{x}\right) \psi_{i},\left(1-\chi_{x}\right) \psi_{j}\right\rangle=\left\langle\psi_{i}, \psi_{j}\right\rangle-\left\langle\left(1-\chi_{x}\right) \psi_{i}, \psi_{j}\right\rangle=\left\langle\chi_{x} \psi_{i}, \psi_{j}\right\rangle .
\end{aligned}
$$


Hence

$$
R_{i j}^{1}(x)=\left\langle\chi_{x} \phi_{i}, \phi_{j}\right\rangle=R_{i j}^{2}(x)=\left\langle\chi_{x} \psi_{i}, \psi_{j}\right\rangle .
$$

We denote $R(x)=R^{1}(x)=R^{2}(x)$. We can pick $\epsilon$ small so that $R^{-1}(x)$ is uniformly bounded for $x \in[0, \epsilon]$.

Recall that $\mu_{j}=\frac{n^{2}}{4}-\lambda_{j}^{2}$ is the eigenvalue corresponding to $\phi_{j}$, as defined in (8.34). For $\delta<\epsilon$, the divergence theorem gives

$$
\begin{gathered}
\left\langle\chi_{\delta} v_{1}(z, \lambda), \phi_{j}(z)\right\rangle=\frac{1}{\mu_{j}}\left\langle\chi_{\delta} v_{1}(z, \lambda), \Delta_{g_{1}} \phi_{j}(z)\right\rangle= \\
\frac{1}{\mu_{j}}\left(\frac{n^{2}}{4}+\lambda^{2}\right)\left\langle\chi_{\delta} v_{1}(z, \lambda), \phi_{j}(z)\right\rangle+\frac{1}{\mu_{j}} \int_{M}\left[v_{1}(\delta, y, \lambda) \frac{\partial \phi_{j}}{\partial x}(\delta, y)-\frac{\partial v_{1}}{\partial x}(\delta, y, \lambda) \phi_{j}(\delta, y)\right] \frac{\sqrt{h}(\delta, y)}{\delta^{n-1}} d y .
\end{gathered}
$$

Doing the same computation for $\left\langle\chi_{\delta} v_{2}, \psi_{j}\right\rangle$, using that $\mu_{j}=\frac{n^{2}}{4}-\lambda_{j}^{2}$ and (8.43), we obtain

$$
\begin{aligned}
& \left\langle\chi_{\delta} v_{1}(z, \lambda), \phi_{j}(z)\right\rangle=-\frac{1}{\lambda_{j}^{2}+\lambda^{2}} \int_{M}\left[v_{1}(\delta, y, \lambda) \frac{\partial \phi_{j}}{\partial x}(\delta, y)-\frac{\partial v_{1}}{\partial x}(\delta, y, \lambda) \phi_{j}(\delta, y)\right] \frac{\sqrt{h}(\delta, y)}{\delta^{n-1}} d y, \\
& \left\langle\chi_{\delta} v_{2}(z, \lambda), \psi_{j}(z)\right\rangle=-\frac{1}{\lambda_{j}^{2}+\lambda^{2}} \int_{M}\left[v_{2}(\delta, y, \lambda) \frac{\partial \phi_{j}}{\partial x}(\delta, y)-\frac{\partial v_{2}}{\partial x}(\delta, y, \lambda) \phi_{j}(\delta, y)\right] \frac{\sqrt{h}(\delta, y)}{\delta^{n-1}} d y .
\end{aligned}
$$

We conclude from (8.50) that there exists $K_{0}(\delta)>0$, such that

$$
\left|F^{1}(\delta, \lambda)-F^{2}(\delta, \lambda)\right|<\frac{K_{0}(\delta)}{\lambda^{2}}\left[\sup _{M}\left|v_{1}(\delta, y, \lambda)-v_{2}(\delta, y, \lambda)\right|+\sup _{M}\left|\frac{\partial v_{1}}{\partial x}(\delta, y, \lambda)-\frac{\partial v_{2}}{\partial x}(\delta, y, \lambda)\right|\right] .
$$

This and (8.49) imply that there exists $K_{1}(\delta)>0$, such that

$$
\begin{gathered}
\sup _{M}\left|T_{1} v_{1}(\delta, y, \lambda)-T_{2} v_{2}(\delta, y, \lambda)\right| \leq \\
\frac{K_{1}(\delta)}{\lambda^{2}}\left[\sup _{M}\left|v_{1}(\delta, y, \lambda)-v_{2}(\delta, y, \lambda)\right|+\sup _{M}\left|\frac{\partial v_{1}}{\partial x}(\delta, y, \lambda)-\frac{\partial v_{2}}{\partial x}(\delta, y, \lambda)\right|\right] .
\end{gathered}
$$

Setting $x=\delta$ in (8.48) and using (8.52) we get

$$
\sup _{M}\left|v_{1}(\delta, y, \lambda)-v_{2}(\delta, y, \lambda)\right| \leq \frac{K_{1}(\delta)}{\lambda^{2}}\left[\sup _{M}\left|v_{1}(\delta, y, \lambda)-v_{2}(\delta, y, \lambda)\right|+\sup _{M}\left|\frac{\partial v_{1}}{\partial x}(\delta, y, \lambda)-\frac{\partial v_{2}}{\partial x}(\delta, y, \lambda)\right|\right] .
$$

To estimate the terms involving $\frac{\partial v_{j}}{\partial x}(\delta, y, \lambda)$, we differentiate [8.48) in $x$. We get that

$$
\frac{\partial v_{1}}{\partial x}(x, y, \lambda)-\frac{\partial v_{2}}{\partial x}(x, y, \lambda)=\frac{\partial T_{1} v_{1}}{\partial x}(x, y, \lambda)-\frac{\partial T_{2} v_{2}}{\partial x}(x, y, \lambda) \text {. }
$$

Since $\Phi=\Psi$ for $x<\epsilon$, we have $T_{j} v_{j}(x, y, \lambda)=R^{-1}(x) F^{j}(x, \lambda) \cdot \Phi(x, y)$, and thus

$$
\begin{gathered}
\frac{\partial}{\partial x} T_{j} v_{j}(x, y, \lambda)= \\
{\left[\frac{d}{d x} R^{-1}(x)\right] F^{j}(x, \lambda) \cdot \Phi(x, y)+R^{-1}(x) \frac{d}{d x} F^{j}(x, \lambda) \cdot \Phi(x, y)+R^{-1}(x) F^{j}(x, \lambda) \cdot \frac{\partial \Phi}{\partial x}(x, y) .}
\end{gathered}
$$

The first and the third terms are much like $T_{j} v_{j}(z, \lambda)$, and because of (8.51), satisfy an estimate like (8.52). The second term has to be considered separately. Notice that

$$
\left(\frac{d}{d x} F^{j}(x, \lambda)\right)^{T}=\left(\frac{d}{d x}\left\langle\chi_{x} v_{j}, \phi_{1}\right\rangle, \ldots \frac{d}{d x}\left\langle\chi_{x} v_{j}, \phi_{L}\right\rangle\right)
$$

and

$$
\left.\frac{d}{d x}\left\langle\chi_{x} v_{j}, \phi_{k}\right\rangle\right|_{x=\delta}=-\frac{1}{\delta^{n+1}} \int_{M} v_{j}(\delta, y, \lambda) \phi_{k}(\delta, y, \lambda) \sqrt{h}(\delta, y, \lambda) d y
$$


Then

$$
\left.\frac{d}{d x}\left(\left\langle\chi_{x} v_{1}, \phi_{k}\right\rangle-\left\langle\chi_{x} v_{2}, \phi_{k}\right\rangle\right)\right|_{x=\delta}=-\frac{1}{\delta^{n+1}} \int_{M}\left(v_{1}(\delta, y, \lambda)-v_{2}(\delta, y, \lambda)\right) \phi_{k}(\delta, y, \lambda) \sqrt{h}(\delta, y, \lambda) d y .
$$

Then there exists $K_{2}(\delta)$ such that

$$
\left|\frac{d}{d x} F^{1}(\delta, \lambda)-\frac{d}{d x} F^{2}(\delta, \lambda)\right| \leq \frac{K_{2}(\delta)}{\lambda^{2}} \sup _{M}\left|v_{1}(\delta, y, \lambda)-v_{2}(\delta, y, \lambda)\right| .
$$

So from (8.51), 8.54), 8.55) and 8.57) we find that there exists $K_{3}(\delta)$ such that

$$
\sup _{M}\left|\frac{\partial v_{1}}{\partial x}(\delta, y, \lambda)-\frac{\partial v_{2}}{\partial x}(\delta, y, \lambda)\right| \leq \frac{K_{3}(\delta)}{\lambda^{2}}\left[\sup _{M}\left|v_{1}(\delta, y, \lambda)-v_{2}(\delta, y, \lambda)\right|+\sup _{M}\left|\frac{\partial v_{1}}{\partial x}(\delta, y, \lambda)-\frac{\partial v_{2}}{\partial x}(\delta, y, \lambda)\right|\right] .
$$

So we conclude from (8.53) and (8.58) that

$$
\begin{gathered}
\sup _{M}\left|v_{1}(\delta, y, \lambda)-v_{2}(\delta, y, \lambda)\right|+\sup _{M}\left|\frac{\partial v_{1}}{\partial x}(\delta, y, \lambda)-\frac{\partial v_{2}}{\partial x}(\delta, y, \lambda)\right| \leq \\
\frac{K_{1}(\delta)+K_{3}(\delta)}{\lambda^{2}}\left[\sup _{M}\left|v_{1}(\delta, y, \lambda)-v_{2}(\delta, y, \lambda)\right|+\sup _{M}\left|\frac{\partial v_{1}}{\partial x}(\delta, y, \lambda)-\frac{\partial v_{2}}{\partial x}(\delta, y, \lambda)\right|\right]
\end{gathered}
$$

Taking $\Lambda^{2}>K_{1}(\delta)+K_{3}(\delta)$, equation (8.47) follows. This ends the proof of the Proposition.

As in the case of no eigenvalues, this can be used to show that the graphs of the Calderón projectors satisfy $C_{1, \lambda}=C_{2, \lambda}, \lambda \in \mathbb{R} \backslash 0$ and by continuity of $C_{j, \lambda}$, if $\lambda \in \mathbb{R}$. However, in this case, the operators $\Delta_{g_{j}}-\frac{n^{2}}{4}, j=1,2$, are not positive, and this only determines the Neumann eigenvalues, and the traces of the corresponding eigenfunctions, of $\Delta_{g_{j}}$ for eigenvalues $\nu_{j} \geq \frac{n^{2}}{4}$. But since the set $J=\left\{j: \nu_{j} \leq \frac{n^{2}}{4}\right\}$ is finite, we may again apply Proposition 8.11 this time with $J \neq \emptyset$.

Observe that for $\delta<\epsilon$ fixed, (8.47), and the density of $v_{j}(\lambda, z), j=1,2$, imply that $C_{1, \lambda}=C_{2, \lambda}$, $\lambda^{2}>\Lambda^{2}$ in $X_{j, \delta}$. This gives the spectral data with eigenvalues $\nu_{j}>\Lambda$ and we could apply Proposition 8.11 already at this stage.

Then Proposition 8.11 combined Proposition 8.2 proves Theorem 8.1 Again, we remark that the method of proof of Proposition 8.11 guarantees that the map is $C^{\infty}$.

\section{ACKnowledgments}

This paper is dedicated to the memory of F.G. Friedlander, who started the study of radiation fields and whose ideas were often used here. I also had the pleasure of having several discussions about the subject with him.

I thank R. Melrose, who was a student of Friedlander, for bringing the paper 14 to my attention and for several instructive conversations. I also thank C. Guillarmou, Y. Kurylev, R. Mazzeo , P. Stefanov, S. Tang and J. Wunsch for useful conversations and for comments on the paper.

I am very grateful to the referees for carefully reading the manuscript. They found several mistakes in previous versions, and made numerous suggestions to improve the exposition.

Part of this work was carried out while I was visiting the Universidade Federal de Pernambuco, Brazil. I thank them, and especially Prof. Paulo Santiago, for their hospitality.

\section{REFERENCES}

[1] S. Alinhac. Unicité du problème de Cauchy pour des opérateurs du second ordre à symboles réels. Ann. Inst. Fourier, Grenoble, 34, 2, 89-109, (1984)

[2] S. Agmon. A representation theorem for solutions of Schrödinger type equations on non-compact Riemannian manifolds. Astérisque, 210, 13-26, (1992) 
[3] S. Agmon. A representation theorem for solutions of the Helmholtz equation on the hyperbolic space. Partial differential equations and related subjects (Trento,1990). Pitman Res. Notes Math. Ser., 269, 1-20.

[4] M. Belishev. Boundary control in reconstruction of manifolds and metrics. Inverse Problems, 13 no. 5, R1-R45. (1997)

[5] M. Belishev and Y. Kurylev. To the reconstruction of a Riemannian manifold via its spectral data (BC-method). Comm. in P.D.E. 17 no. 5 \& 6, 767-804, (1992)

[6] D. Borthwick and P. Perry. Scattering poles for asymptotically hyperbolic manifolds. Trans. A.M.S. 354 no. 3, 12151231, (2002).

[7] E.B.Davies. Spectral theory and differential operators. Cambridge Univ. Press, (1995).

[8] L. Fadeev and B. Pavlov. Scattering theory and automorphic functions. Proc. Steklov Inst. Math. 27, 163-193, (1972)

[9] C. Fefferman and C.R, Graham. Q-curvature and Poincaré metrics. Math. Res. Lett. 9, no.2-3, 139-151, (2002)

[10] F.G. Friedlander. On the radiation field of pulse solutions of the wave equation. Proc. Roy. Soc. Ser. A. 269, 53-65, (1962)

[11] F.G. Friedlander. On the radiation field of pulse solutions of the wave equation II. Proc. Roy. Soc. Ser. A. 279, 386-394, (1963)

[12] F.G. Friedlander. On the radiation field of pulse solutions of the wave equation III. Proc. Roy. Soc. Ser. A. 299, 386-394, (1965)

[13] F.G. Friedlander. Radiation fields and hyperbolic scattering theory. Math. Proc. Camb. Phil. Soc., 88, 483-515, (1980)

[14] F.G. Friedlander. Notes on the wave equation on asymptotically Euclidean manifolds. J. of Func. Anal. 184, no. 1, $1-18,(2001)$

[15] C.R. Graham. Volume and area renormalization for conformally compact Einstein metrics. Rend. Circ. mat. Palermo 2 Suppl. 63, 31-42, (2000)

[16] C.R. Graham and M. Zworski, Scattering matrix in conformal geometry. Inventiones Math. 152 no. 1, 89-118, (2003)

[17] C. Guillarmou. Meromorphic properties of the resolvent on asymptotically hyperbolic manifolds. Preprint 2003.

[18] L. Guillopé. Fonctions zêta de Selberg et surfaces de géométrie finie. Advanced Studies in Pure Math. 21, 33-70, (1992)

[19] L. Guillopé, M. Zworski. Polynomial bounds on the number of resonances for some complete spaces of constant negative curvature near infinity. Asymptotic Analysis 11, 11-22, (1995)

[20] L. Guillopé, M. Zworski. Scattering asymptotics for Riemann surfaces. Ann. of Math. 145, 597-660, (1997)

[21] S. Helgason. Functions on symmetric spaces. Harmonic Analysis on homogeneous spaces. Proc. Symposia in Pure Math., 26 Amer. Math. Soc., 101-146, (1973).

[22] S. Helgason. Wave equations on homogeneous spaces. Lecture Notes in Math. 1077, 254-287, Springer Verlag (1984)

[23] S. Helgason. Support theorems in integral geometry and their applications. Differential geometry: geometry in mathematical physics and related topics. Proc. Sympos. Pure Math. 54, part 2, 317-323. Amer. Math. Soc., Providence, RI, (1993)

[24] S. Helgason. The Radon Transform. 2nd Edition Vol. 5, progress in mathematics, Birkhäuser, (1999)

[25] L. Hörmander. Linear partial differential operators. Grundlehren Math. Wiss. 116, Springer Verlag, (1963)

[26] L. Hörmander. The analysis of linear partial differential operators. Vol 1-4, Grundlehren Math. Wiss. 256, Springer Verlag, (1983)

[27] L. Hörmander. A uniqueness theorem for second order hyperbolic differential equations. Comm. in P.D.E. 17, 699-714 (1992)

[28] M.S. Joshi and A. Sá Barreto. Inverse scattering on asymptotically hyperbolic manifolds. Acta Mathematica, 184, 41-86, (2000)

[29] A. P. Katchalov and Ya. V. Kurylev. Incomplete spectral data and the reconstruction of a Riemannian manifold. J. Inv. Ill-Posed problems, 1 No. 2, 141-153 (1993)

[30] A. Katchalov and Y. Kurylev. Multidimensional inverse problem with incomplete boundary spectral data. Comm. in P.D.E. 23 No. 1\& 2, 55-95 (1998).

[31] A. Katchalov, Y. Kurylev and M. Lassas. Inverse boundary spectral problems. Chapman \& hall/CRC, 2001.

[32] I.A. Kiprijanov, L.A. Ivanov. The Euler Poisson-Darboux equation in a Riemannian space. Soviet Math. Dokl. 24 No. 2, 331-335, (1981)

[33] P. Lax. The Radon transform and translation representation. Journ. Evol. Equ. 1, 311-323, (2001).

[34] P. Lax and R. Phillips. Translation representation for automorphic solutions of the wave equation in non-Euclidean spaces. I. Comm. Pure Appl. Math. 37 no. 3, 303-328. (1984).

[35] P. Lax and R. Phillips. Scattering theory. Academic Press, revised edition, (1989)

[36] P. Lax and R. Phillips. Translation representation for the solutions of the non-Euclidean wave equation. Comm. on Pure and Appl. Math. 32, 617-667, (1979) 
[37] P. Lax and R. Phillips. Scattering theory for automorphic forms. Ann. of Math. Studies No. 87 Princeton Univ. Press, (1976)

[38] N. Lerner and L. Robbiano. Unicité de Cauchy pour des opérateurs de type principal. J. Analyse Math. 44, 32-66, $(1984 / 85)$.

[39] R. Mazzeo. The Hodge cohomology of a conformally compact metric. J. Diff. Geom. 28, 309-339, (1988)

[40] R. Mazzeo. Unique continuation at infinity and embedded eigenvalues for asymptotically hyperbolic manifolds. Am. J. Math. 113 25-45, (1991)

[41] R. Mazzeo. Elliptic theory of differential edge operators I. Comm. in P.D.E. 16(10), 1615-1664, (1991)

[42] R. Mazzeo. Remarks on a paper of Friedlander concerning inequalities between Neumann and Dirichlet eigenvalues. Intern. Math. Res. Notices. 4, 41-48, (1991)

[43] R. Mazzeo and R.B. Melrose. Meromorphic extension of the resolvent on complete spaces with asymptotically constant negative curvature. J. Func. Anal. 108, 260-310, (1987)

[44] R.B. Melrose. Geometric scattering theory. Stanford Lectures, Cambridge Univ. Press, (1995)

[45] P. Perry. The Laplace operator on a hyperbolic manifold, II. Eisenstein series and the scattering matrix. J. Reine Angew. Math., 398, 67-91, (1989)

[46] L. Robbiano. Théorème d'unicité adapté au contrôle des solutions des problèmes hyperboliques. Comm. in P.D.E 16, 789-800, (1991)

[47] L. Robbiano and C. Zuilly. Uniqueness in the Cauchy problem for operators with partially holomorphic coefficients. Invent. Math. 131, 493-539, (1998)

[48] A. Sá Barreto. Radiation fields on asymptotically Euclidean manifolds. Comm. in P.D.E. 28 , Nos. 9 \& 10, 1661-1673, (2003)

[49] D. Tataru. Unique continuation for solutions to PDE's: between Hörmander's theorem and Holmgren's theorem. Comm. in P.D.E. 20, 855-884, (1995)

[50] D. Tataru. Unique continuation for operators with partially analytic coefficients. J. Math. Pures Appl. 78, 505-521, (1999)

[51] M. E. Taylor. Pseudodifferential operators. Princeton Univ. Press, 1981.

[52] G. Uhlmann. Scattering by a metric. Scattering and Inverse Scattering in Pure and Applied Sciences, 1668-1677, (2002)

Department of Mathematics, Purdue University, 150 North University Street, West Lafayette IN 47907, USA

E-mail address: sabarre@math.purdue.edu 